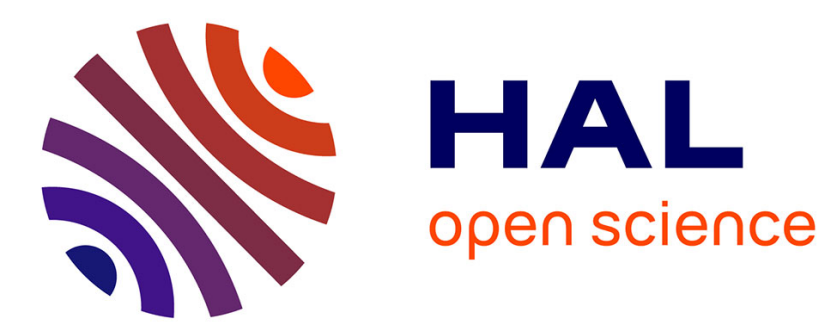

\title{
Augmented Galerkin Schemes for the Numerical Solution of Scattering by Small Obstacles.
}

Xavier Claeys, Francis Collino

\section{To cite this version:}

Xavier Claeys, Francis Collino. Augmented Galerkin Schemes for the Numerical Solution of Scattering by Small Obstacles.. [Research Report] RR-6195, INRIA. 2007. inria-00148395v2

\section{HAL Id: inria-00148395 \\ https://hal.inria.fr/inria-00148395v2}

Submitted on 23 May 2007

HAL is a multi-disciplinary open access archive for the deposit and dissemination of scientific research documents, whether they are published or not. The documents may come from teaching and research institutions in France or abroad, or from public or private research centers.
L'archive ouverte pluridisciplinaire HAL, est destinée au dépôt et à la diffusion de documents scientifiques de niveau recherche, publiés ou non, émanant des établissements d'enseignement et de recherche français ou étrangers, des laboratoires publics ou privés. 
INSTITUT NATIONAL DE RECHERCHE EN INFORMATIQUE ET EN AUTOMATIQUE

\title{
Augmented Galerkin Schemes for the Numerical Solution of Scattering by Small Obstacles.
}

\author{
Xavier Claeys — Francis Collino
}

$\mathbf{N}^{\circ} 6195$

Mai 2007

Thème NUM

apport

de recherche 



\title{
RINRIA
}

\section{Augmented Galerkin Schemes for the Numerical Solution of Scattering by Small Obstacles.}

\author{
Xavier Claeys , Francis Collino \\ Thème NUM - Systèmes numériques \\ Projets POems \\ Rapport de recherche $\mathrm{n}^{\circ} 6195$ - Mai 2007 - 47 pages
}

\begin{abstract}
:
In the context of electromagnetic wave propagation, we wish to adress the scattering problem from perfectly conducting thin wires. For numerical simulations, assuming their thickness to be much smaller than the wavelength of the incident field, it is not possible to take these obstacles into account without encountering problems of numerical locking.

The Holland model, widely used in finite difference schemes, provides a pragmatic solution to this problem, by modifying the numerical scheme on vertices located in the neighbourhood of the wires. So far this model has not received any real theoretical justification, and involves a parameter, named lineic inductance, which is to be chosen on the basis of heuristic considerations. We are interested in the simplified problem of a bidimensional acoustic wave propagation in a medium including a small obstacle with homogeneous Dirichlet boundary condition. We present and analyse a numerical scheme suitable for finite elements that does not suffer from numerical locking, and takes the presence of the small obstacle into account. It is based on a combination between the fictitious domain method and matched asymptotic expansions. This results into a systematic generalization to the Holland model including an automatic computation of the lineic inductance. Our analysis leads to the first (to our knowledge) justification of this type of model.
\end{abstract}

Key-words: Wire, small obstacle, scattering, finite element, Holland model, singular function method, Helmholtz equation

Unité de recherche INRIA Rocquencourt

Domaine de Voluceau, Rocquencourt, BP 105, 78153 Le Chesnay Cedex (France)

Téléphone : +33139635511-Télécopie : +33139635330 


\section{Augmented Galerkin Schemes for the Numerical Solution of Scattering by Small Obstacles.}

\section{Résumé :}

Dans le contexte de la propagation des ondes electromagnétiques, nous nous intéressons au problème de diffraction par des fils minces parfaitement conducteurs. Si l'on suppose que leur épaisseur est bien plus petite que la longueur d'onde caractéritique de l'onde incidente, il n'est pas posible de prendre en compte des fils minces sans faire face à un problème de verrouillage numérique.

Le modèle de Holland, largement utilisé dans les codes différences finis, fournit une solution pragmatique à ce problème, en modifiant le schéma numérique sur quelques noeuds du maillage avoisinant les fils. Jusqu'à présent ce modèle n'a pas reçu de justification théorique solide, et il implique un paramètre appelé l'inductance linéique, qu'il doit être choisi suivant des considértions heuristiques.

Nous nous intéressons ici au problème modèle de la diffraction acourtique par un petit obstacle, avec condition de Dirichlet au bord, en deux dimensions dans un milieu homogène. Nous présentons et analysons un schéma numérique qui est compatible avec les méthodes éléments finis standards (sans raffinement de maillage) et ne souffre de verrouillage numérique. Ce schéma mélange des techniques d'analyse asymptotique avec une formulation de type domaine fictif. Suivant les résultats que nous démontrons sur ce schéma, nous aboutissons à une généralisation du modèle de Holland et à un calcul automatique de l'inductance linéique. Notre analyse amène, à notre connaissance, à la première justification théorique de ce type de modèle.

Mots-clés : Fil, petit obstacle, diffraction, éléments finis, modèle de Holland, méthode de la fonction singulière, équation de Helmholtz 


\section{Contents}

1 Introduction 4

$2 \quad$ Exact and simplified problems

2.1 Preliminarv results of asvmptotic analvsis $\ldots \ldots \ldots \ldots \ldots \ldots$

2.2 Simplified problem . . . . . . . . . . . . . . . . . . . 10

$3 \quad$ Discretization of the simplified problem 12

3.1 Solution of the simplified problem using standard discretization . . . . . . . . . . 13

3.1 .1 Formulation . . . . . . . . . . . . . . . . . . . 13

$3.1 .2 \quad$ Stability studv . . . . . . . . . . . . . . . . . . 13

3.2 Numerical experiments for the simplified scheme . . . . . . . . . . . . . . 15

4 Augmented Galerkin scheme 18

4.1 Presentation and first analvsis of the method . . . . . . . . . . . . . . 18

4.2 Global consistency . . . . . . . . . . . . . . . . . . . . . . . . . 19

4.3 Error estimate awav from the obstacle . . . . . . . . . . . . . . . . . . . 21

4.4 Numerical experiments on the augmented formulation . . . . . . . . . . . . 21

5 Study of the additional shape function $\quad 24$

5.1 Global estimate . . . . . . . . . . . . . . . . . . . . . . 25

5.2 Local estimate . . . . . . . . . . . . . . . . . . . . . . 26

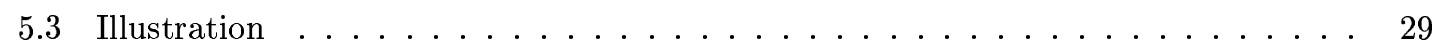

5.4 Decomposition of the additional shape function $\ldots \ldots \ldots \ldots \ldots$

5.4 .1 Numerical Dirac Function . . . . . . . . . . . . . . . . . . . . . . . . . 30

5.4 .2 Regularized Potential $\ldots \ldots \ldots$. . . . . . . . . . . . . . 31

6 The Holland model 33

6.1 Presentation of the Holland Model . . . . . . . . . . . . . . . . . . . . . . 33

6.2 Relation between augmented Galerkin scheme and Holland scheme . . . . . . . . . 35

6.3 Theoritical Expression of the Lineic Inductance . . . . . . . . . . . . . . . . . 36

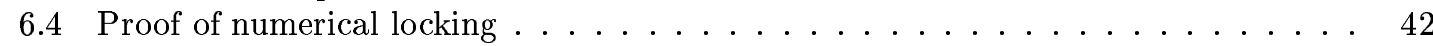

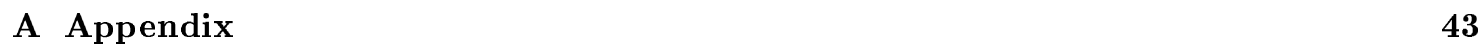

A.1 Nitsche and Schatz' theorem . . . . . . . . . . . . . . . . . . . 43

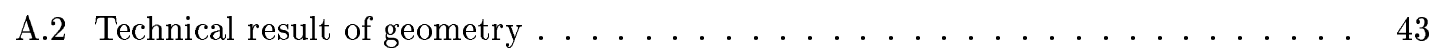

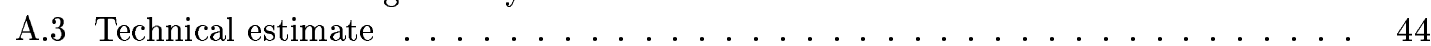




\section{Introduction}

The general context of this work is the simulation of wave propagation in media including small obstacles. In order to perform such a computation, a first approach consists in applying standard techniques (finite elements for instance) everywhere in the domain of computation. Because of their size, small obstacles require mesh refinement in order to be taken into account: time and memory used for these computations significantly increase because of these details, which implies bad computational efficiency.

In this work we propose a way to bypass such a refinement. We choose a simplified context and consider a 2-D geometry where Helmholtz equation has to be solved. This work is a first step toward efficient methods able to take into account perfectly conducting thin wires in simulations of electromagnetic wave propagation.

Our method consists, on the basis of an asymptotic analysis, to augment the approximation space with an additional shape function containing a singularity adapted to the small obstacle. This method is very close to singular complement methods (or singular function methods) used in electromagnetics in order to obtain consistency when computational domains contain reentrant corners. There exists a vast literature about this subject, see for example [2], [9].

Let $D(0, R)$ be the disk of radius $R$ with center 0 and $\Omega_{\text {obs }}$ be an open subset of $D(0, R)$ that does not intersect some neighborhood of the origin. We note $\Gamma_{R}=\partial D(0, R)$. The domain on which this study takes place is the polygonal open set $\Omega_{R}$ approaching $D(0, R) \backslash \overline{\Omega_{o b s}}$. We suppose that $\Omega_{\varepsilon}^{i}$ is a small obstacle with boundary $\Gamma^{\varepsilon}$ described by the polar equation

$$
\left(\Gamma^{\varepsilon}\right): r=\varepsilon \gamma(\theta) .
$$

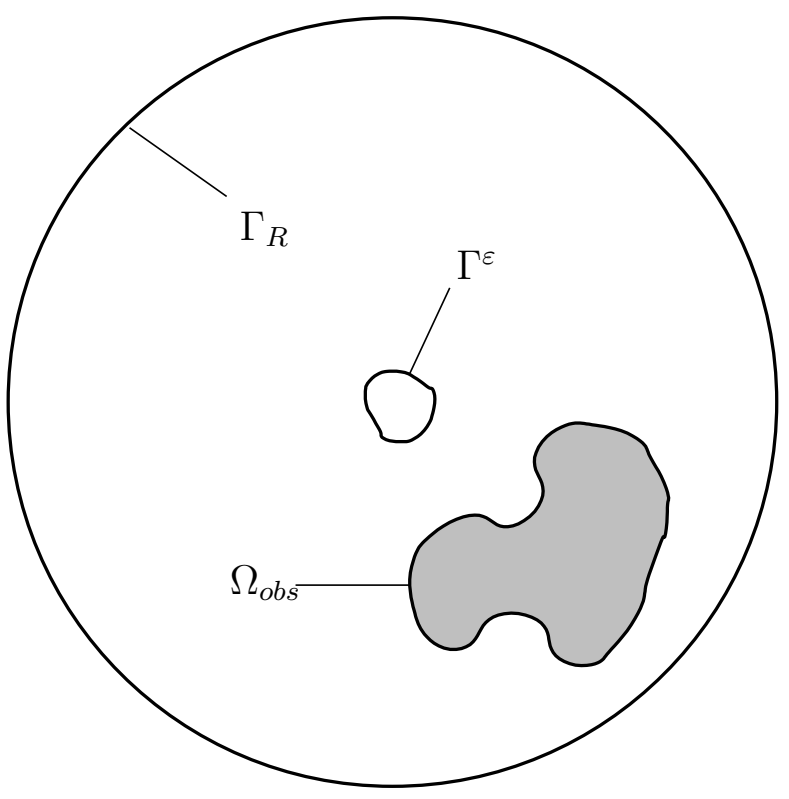

Figure 1: Geometry of the problem

The outline of this work is as follows. We consider first a problem called "exact" that takes the form of a saddle point problem ( $a, b^{\varepsilon}, V$ and $Q_{\text {ex }}$ will be defined later).

$$
\left\{\begin{array}{l}
\text { Find }\left(u^{\varepsilon}, p^{\varepsilon}\right) \in V \times Q_{\mathrm{ex}} \text { such that } \\
a\left(u^{\varepsilon}, v\right)+b^{\varepsilon}\left(v, p^{\varepsilon}\right)=\langle f, v\rangle_{V, V^{\prime}} \quad \forall v \in V, \\
b^{\varepsilon}\left(u^{\varepsilon}, q\right)=0 \quad \forall q \in Q_{\mathrm{ex}} .
\end{array}\right.
$$


In this problem, we take into account the presence of a small obstacle with diameter $\varepsilon$, so the solution $\left(u^{\varepsilon}, p^{\varepsilon}\right)$ naturally depends on $\varepsilon$. In order to describe this solution, it is possible to take advantage of the fact that $\varepsilon$ is very small by using an asymptotic analysis that we will describe later. On the basis of these results, we propose to replace the initial space of Lagrange multipliers $Q_{\text {ex }}$ by a reduced space $Q_{\text {ap }}$. The solution $\left(\mathfrak{u}^{\varepsilon}, \mathfrak{p}^{\varepsilon}\right)$ of the corresponding new problem will then satisfy

$$
\left\{\begin{array}{l}
\text { Find }\left(\mathfrak{u}^{\varepsilon}, \mathfrak{p}^{\varepsilon}\right) \in V \times Q_{\text {ap }} \text { such that } \\
a\left(\mathfrak{u}^{\varepsilon}, v\right)+b^{\varepsilon}\left(v, \mathfrak{p}^{\varepsilon}\right)=\langle f, v\rangle_{V, V^{\prime}} \quad \forall v \in V, \\
b^{\varepsilon}\left(\mathfrak{u}^{\varepsilon}, q\right)=0 \quad \forall q \in Q_{\text {ap }} .
\end{array}\right.
$$

In order to obtain a numerical solution, we could discretize this problem using the approximation spaces denoted $V^{h}$ and $Q_{\mathrm{ap}}^{h}$ of Lagrange finite elements on a regular mesh. The solution of the corresponding discrete problem $\left(u^{\varepsilon, h}, p^{\varepsilon, h}\right)$ then satisfies

$$
\left\{\begin{array}{l}
\text { Find }\left(u^{\varepsilon, h}, p^{\varepsilon, h}\right) \in V^{h} \times Q_{\mathrm{ap}}^{h} \text { such that } \\
a\left(u^{\varepsilon, h}, v\right)+b^{\varepsilon}\left(v, p^{\varepsilon, h}\right)=\langle f, v\rangle_{V^{h}, V^{h^{\prime}}} \quad \forall v \in V^{h}, \\
b^{\varepsilon}\left(u^{\varepsilon, h}, q\right)=0 \quad \forall q \in Q_{\mathrm{ap}}^{h} .
\end{array}\right.
$$

This problem depends on two small parameters, $\varepsilon$ and $h$. It is thus possible to consider several regimes: $h$ goes to 0 faster than $\varepsilon$, or $\varepsilon$ goes to 0 faster than $h$. We will be interested in the second regime, when the mesh is coarse compared to the small obstacle. In such a situation, standard numerical approximation suffers from numerical locking, in the sense that $u^{\varepsilon, h}$ is a bad approximation of $\mathfrak{u}^{\varepsilon}$ if $\varepsilon$ is too small compared to $h$. We will prove that, under the condition $\varepsilon<\kappa h$ for a given fixed $\kappa>0$, there exists another fixed $\kappa>0$ such that $\left\|\mathfrak{u}^{\varepsilon}-u^{\varepsilon, h}\right\|_{V}>\kappa / \ln 1 / \varepsilon$. This means that under the condition that $\varepsilon$ goes to 0 as fast as $h$ then standard approximation converges very slowly. In order to cure this problem, we introduce an additional shape function $\psi^{\varepsilon}$ that has to reproduce the singular asymptotic behavior of the exact solution. Indeed, this behavior is the reason of the previous lack of consistency. Thus, we consider the new approximation space $V_{\mathrm{e}}^{h}=V^{h} \oplus \operatorname{span}\left\{\psi^{\varepsilon}\right\}$ and the solution $\left(u^{\varepsilon, h}, p^{\varepsilon, h}\right)$ of the corresponding discrete problem

$$
\left\{\begin{array}{l}
\text { Find }\left(u^{\varepsilon, h}, p^{\varepsilon, h}\right) \in V_{\mathrm{e}}^{h} \times Q_{\mathrm{ap}}^{h} \text { such that } \\
a\left(u^{\varepsilon, h}, v\right)+b^{\varepsilon}\left(v, p^{\varepsilon, h}\right)=\langle f, v\rangle_{V^{h}, V^{h^{\prime}}} \quad \forall v \in V_{\mathrm{e}}^{h}, \\
b^{\varepsilon}\left(u^{\varepsilon, h}, q\right)=0 \quad \forall q \in Q_{\mathrm{ap}}^{h} .
\end{array}\right.
$$

Using this new discretization, the approximation becomes consistent. Indeed, we will show that there exists $\kappa>0$ independent of $\varepsilon$ and $h$ such that

$$
\left\|u^{\varepsilon}-u^{\varepsilon, h}\right\|_{H^{1}\left(\Omega_{R}\right)} \leqslant \kappa \sqrt{\ln 1 / \varepsilon}\left(\varepsilon^{1 / 2}+h\right) .
$$

In the last part of this document, we will get back to the Holland scheme, often used in practice. It takes the form

$$
\left\{\begin{array}{l}
\text { Find }\left(u_{\mathbf{h}}^{\varepsilon, h}, p_{\mathbf{h}}^{\varepsilon, h}\right) \in V^{h} \times Q_{\mathrm{ap}}^{h} \text { such that } \\
a\left(u_{\mathbf{h}}^{\varepsilon, h}, v\right)+b^{\varepsilon}\left(v, p_{\mathbf{h}}^{\varepsilon, h}\right)=\langle f, v\rangle_{V^{h}, V^{h^{\prime}}} \quad \forall v \in V^{h}, \\
b^{\varepsilon}\left(u_{\mathbf{h}}^{\varepsilon, h}, q\right)-L_{\mathbf{h}}^{\varepsilon, h} c\left(p_{\mathbf{h}}^{\varepsilon, h}, q\right)=0 \quad \forall q \in Q_{\mathrm{ap}}^{h} .
\end{array}\right.
$$

In this last scheme, $L_{\mathbf{h}}^{\varepsilon, h}$ is a parameter that is not a priori known and that has to be chosen on the basis of empirical considerations. We will show that Scheme (1.4) is related to Holland's Scheme 
(1.5). In particular, this will lead us to a theoretical approach of Holland schemes providing the required value for the parameter $L_{\mathbf{h}}^{\varepsilon, h}$. This is, to the best of our knowledge, the first serious theoretical development about the Holland model : so far, the problem of finding the good value for the Holland parameter in an arbitrary mesh had remained an opened question.

\section{Exact and simplified problems}

We are presently interested in an acoustic diffraction problem. More precisely, we want to solve in $\Omega_{R} \backslash \Omega_{\varepsilon}^{i}$ the Helmholtz equation $\Delta u+k^{2} u=f$ with outgoing radiation condition. Moreover, we impose a Dirichlet condition on the boundary $\Gamma^{\varepsilon}$ of the small obstacle. We consider an homogeneous condition on the small obstacle, but it seems that a similar treatment can be adopted for non-homogeneous boundary conditions. The standard variational formulation of this problem is the following.

$$
\left\{\begin{array}{l}
\text { Find } \quad u \in H^{1}\left(\Omega_{R} \backslash \overline{\Omega_{\varepsilon}^{i}}\right) \text { s.t } u_{\mid \partial \Omega_{o b s} \cup \Gamma^{\varepsilon}}=0 \quad \text { and } \forall v \in H^{1}\left(\Omega_{R} \backslash \overline{\Omega_{\varepsilon}^{i}}\right) \text { s.t } \quad v_{\mid \partial \Omega_{o b s} \cup \Gamma^{\varepsilon}}=0 \\
\int_{\Omega_{R} \backslash \overline{\Omega_{\varepsilon}^{i}}} \nabla u \cdot \nabla \bar{v}-k^{2} \int_{\Omega_{R} \backslash \overline{\Omega_{\varepsilon}^{i}}} u \bar{v}+\int_{\Gamma_{R}} \bar{v} T_{R} u=\int_{\Omega_{R} \backslash \overline{\Omega_{\varepsilon}^{i}}} f \bar{v} .
\end{array}\right.
$$

Here $T_{R}$ refers to the Dirichlet-to-Neumann operator for the 2-D Helmholtz equation. $T_{R}$ admits an analytical expression that can be decomposed using Fourier expansion,

$$
T_{R} u=\sum_{n \in \mathbb{Z}} u_{n} k \frac{H_{n}^{(1)^{\prime}}(k R)}{H_{n}^{(1)}(k R)} e^{i n \theta} \quad \text { where } \quad u_{n}=\frac{1}{2 \pi} \int_{0}^{2 \pi} u(R, \theta) e^{-i n \theta} d \theta
$$

It is a classical result that $T_{R}$ is a continuous linear operator from $H^{1 / 2}\left(\Gamma_{R}\right)$ onto $H^{-1 / 2}\left(\Gamma_{R}\right)$. Note that we want to consider arbitrary meshes, not necessarily adapted to the small obstacle $\Omega_{\varepsilon}^{i}$. This is the reason why we propose to use a fictitious domain formulation (see [5]) for this problem, extending the solution by 0 inside $\Omega_{\varepsilon}^{i}$ (which still allows $H^{1}$ regularity for the solution). Thus, the extended solution is sought in the space

$$
V=\left\{v \in H^{1}\left(\Omega_{R}\right) \quad \text { s.t } \quad v_{\mid \partial \Omega_{o b s}}=0\right\} .
$$

The usual norm $\|v\|_{V}^{2}=\|v\|_{L^{2}\left(\Omega_{R}\right)}^{2}+\|\nabla v\|_{L^{2}\left(\Omega_{R}\right)}^{2}$ and scalar product $(u, v)=\int_{\Omega_{R}} u v+\int_{\Omega_{R}} \nabla u \cdot \nabla v$, make $V$ a Hilbert space. The initial "exact" problem, equivalent to the previous one, is

$$
\left\{\begin{array}{l}
\text { Find } \quad\left(u^{\varepsilon}, p^{\varepsilon}\right) \in H_{0}^{1}\left(\Omega_{R}\right) \times H^{-1 / 2}\left(\Gamma^{\varepsilon}\right) \quad \text { such that } \\
\int_{\Omega_{R}} \nabla u^{\varepsilon} \cdot \nabla \bar{v}-k^{2} \int_{\Omega_{R}} u^{\varepsilon} \bar{v}+\int_{\Gamma_{R}} \bar{v} T_{R} u^{\varepsilon}+\int_{\Gamma^{\varepsilon}} p^{\varepsilon} \bar{v}=\int_{\Omega_{R}} f \bar{v} \quad \forall v \in H_{0}^{1}\left(\Omega_{R}\right), \\
\int_{\Gamma^{\varepsilon}} q u^{\varepsilon}=0 \quad \forall q \in H^{-1 / 2}\left(\Gamma^{\varepsilon}\right) .
\end{array}\right.
$$

In the second equation, we impose an homogeneous Dirichlet boundary condition on $\Gamma^{\varepsilon}$ using Lagrange multipliers. We rewrite this problem in the more compact way

$$
\left\{\begin{array}{l}
\text { Find }\left(u^{\varepsilon}, p^{\varepsilon}\right) \in V \times Q_{\mathrm{ex}} \text { such that que } \\
a\left(u^{\varepsilon}, v\right)+b^{\varepsilon}\left(v, p^{\varepsilon}\right)=\langle f, v\rangle_{V, V^{\prime}} \quad \forall v \in V \\
b^{\varepsilon}\left(u^{\varepsilon}, q\right)=0 \quad \forall q \in Q_{\mathrm{ex}}
\end{array}\right.
$$


taking

$$
\begin{aligned}
& Q_{\mathrm{ex}}=H^{-1 / 2}\left(\Gamma^{\varepsilon}\right), \\
& a(u, v)=\int_{\Omega_{R}} \nabla u \cdot \nabla \bar{v}-k^{2} \int_{\Omega_{R}} u \bar{v}+\int_{\Gamma_{R}} \bar{v} T_{R} u, \quad \forall u, v \in V, \\
& b^{\varepsilon}(v, q)=\int_{\Gamma^{\varepsilon}} q \bar{v}, \quad \forall(v, q) \in V \times Q_{\mathrm{ex}}, \\
& \langle f, v\rangle_{V, V^{\prime}}=\int_{\Omega_{R}} f \bar{v} .
\end{aligned}
$$

Remind that according to the results of [1, this problem is well posed. Using Green formula, it can be shown that $p^{\varepsilon}$ is the jump of the normal derivative of $u^{\varepsilon}$, which reads

$$
p^{\varepsilon}=\left[\frac{\partial u^{\varepsilon}}{\partial n}\right]_{\mid \Gamma^{\varepsilon}}=\frac{\partial u^{\varepsilon}}{\partial n} \mid \Gamma^{\varepsilon} \quad \text { with } n \text { the outgoing normal vector to } \Omega_{\varepsilon}^{i} .
$$

One of the advantages of this fictitious domain formulation is to provide directly the function $p^{\varepsilon}$ that plays the same role for the Helmholtz equation as the current at the surface of the wire for Maxwell's equations. Since the obstacle $\Omega_{\varepsilon}^{i}$ is small, we encounter the question of the behavior of the couple $\left(u^{\varepsilon}, p^{\varepsilon}\right)$ as $\varepsilon \rightarrow 0$. The next section addresses this question.

\subsection{Preliminary results of asymptotic analysis}

In order to obtain a more explicit description of the solution $u^{\varepsilon}$, we will analyze its behavior as $\varepsilon$ becomes very small. Let us start with some intuitive ideas. When $\varepsilon$ becomes very small, the obstacle $\Omega_{\varepsilon}^{i}$ disappears so it seems reasonable to suppose that $u^{\varepsilon}$ gets closer to the limit "incident field" $u_{0}$, solution to the diffraction problem without any obstacle (we will define it precisely below), i.e. $u^{\varepsilon} \simeq u_{0}$. Actually it is possible to prove that there exists $\kappa>0$ independent of $\varepsilon$ such that

$$
\left\|u^{\varepsilon}-u_{0}\right\|_{H^{1}\left(\Omega_{R}\right)} \leqslant \frac{\kappa}{\sqrt{\ln 1 / \varepsilon}}
$$

Thus, the incident field provides a first approximation of $u^{\varepsilon}$, but not very precise. In order to obtain more precision, we can use the asymptotic analysis that was proposed in [3]. It contains the following result.

\section{Theorem 2.1}

There exists $\kappa>0$ independent of $\varepsilon$ such that for $\varepsilon$ small enough

$$
\left\|u^{\varepsilon}-\widetilde{u}^{\varepsilon}\right\|_{H^{1}\left(\Omega_{R}\right)} \leqslant \kappa \varepsilon^{1 / 2} .
$$

with

$$
\widetilde{u}^{\varepsilon}(\mathbf{x})=\left(1-\chi_{1 / 2}^{\varepsilon}(\mathbf{x})\right)\left(u_{0}(\mathbf{x})+\frac{-\frac{\pi}{2} u_{0}(0)}{\ln \varepsilon-\lambda_{0}} v_{0}(\mathbf{x})\right)+\chi_{1 / 2}^{\varepsilon}(\mathbf{x}) \frac{-2 \pi u_{0}(0)}{\ln \varepsilon-\lambda_{0}} V_{0}^{\varepsilon}(\mathbf{x}) \mathbf{1}_{\Omega_{R} \backslash \bar{\Omega}_{\varepsilon}^{i}}(\mathbf{x})
$$

We immediately remind the definitions necessary for a good comprehension of the above formula.

\section{- The cut-off function $\chi$}

In the rest of this document $\chi: \mathbb{R} \rightarrow \mathbb{R}_{+}$refers to a $C^{\infty}$ function such that $\chi(r)=1$ for $r \leqslant 1 / 2, \chi(r)=0$ for $r \geqslant 1$ and $\chi$ is decreasing. We will write $\chi_{\delta}^{\varepsilon}(\mathbf{x})=\chi\left(|\mathbf{x}| / \varepsilon^{\delta}\right)$, and also $\chi_{0}$ instead of $\chi_{0}^{\varepsilon}$.

- The incident field $u_{0}$ 
$u_{0}$ refers to the incident field defined as the unique function satisfying

$$
\left\{\begin{array}{l}
u_{0} \in H^{1}\left(\Omega_{R}\right) \\
\Delta u_{0}+k^{2} u_{0}=f \quad \text { in } \quad \Omega_{R} \backslash\{0\} \\
u_{0}=0 \text { on } \partial \Omega_{R} \backslash \Gamma_{R} \\
\frac{\partial u_{0}}{\partial n}+T_{R} u_{0}=0 \quad \text { on } \quad \Gamma_{R}
\end{array}\right.
$$

- The far field $v_{0}$

We define the far field as the unique solution of the problem below.

Lemma 2.1 There exists a unique $v_{0} \in H_{\text {loc }}^{1}\left(\Omega_{R} \backslash\{0\}\right)$ satisfying:

$$
\left\{\begin{array}{l}
\Delta v_{0}+k^{2} v_{0}=0 \quad \text { in } \quad \Omega_{R} \backslash\{0\} \\
v_{0}=0 \quad \text { on } \quad \partial \Omega_{R} \backslash \Gamma_{R} \\
\frac{\partial v_{0}}{\partial n}+T_{R} v_{0}=0 \quad \text { on } \quad \Gamma_{R} \\
v_{0}-Y_{0}(k r) \in H^{1}\left(\Omega_{R}\right)
\end{array}\right.
$$

By separation of variables, it is possible to prove that $v_{0}$ admits the unique decomposition

$$
v_{0}=Y_{0}(k|\mathbf{x}|)+\mathcal{L}_{0}^{1}\left(v_{0}\right) J_{0}(k|\mathbf{x}|)+\sum_{|p| \geqslant 1} \mathcal{L}_{p}^{1}\left(v_{0}\right) J_{|p|}(k|\mathbf{x}|) e^{i p \theta}+\sum_{|p| \geqslant 1} \mathcal{L}_{p}^{0}\left(v_{0}\right) Y_{|p|}(k|\mathbf{x}|) e^{i p \theta}
$$

In order to keep notations consistent with [3], We note $J_{0,0}=1$ and $Y_{0,0}=\frac{2}{\pi} \gamma+\frac{2}{\pi} \ln \left(\frac{k}{2}\right)$, where $\gamma=0,57721 \ldots$ is the Euler constant.

Remark: In the case where $\Omega_{R}$ is the disk of center 0 and radius $R$, we have

$$
\begin{aligned}
& v_{0}(\mathbf{x})=-i H_{0}^{(1)}(k|\mathbf{x}|)=-i J_{0}(k|\mathbf{x}|)+Y_{0}(k|\mathbf{x}|) \\
& \mathcal{L}_{0}^{1}\left(v_{0}\right)=1 / i \quad \text { and } \quad \mathcal{L}_{p}^{0}\left(v_{0}\right)=\mathcal{L}_{p}^{1}\left(v_{0}\right)=0 \quad|p|>0
\end{aligned}
$$

\section{- The near field $V_{0}^{\varepsilon}$}

We define this function via a lemma of existence and uniqueness. Remind that $\Gamma^{\varepsilon}=$ $\left\{\mathbf{x}(r, \theta) \in \mathbb{R}^{2} \mid r=\varepsilon \gamma(\theta)\right\}$.

\section{Lemma 2.2}

Define $\Omega_{N}=\left\{\mathbf{x}(r, \theta) \in \mathbb{R}^{2} \mid r>\gamma(\theta) \quad\right\}$. There exists a unique $\mathcal{V}_{0} \in H_{\text {loc }}^{1}\left(\Omega_{N}\right)$ and a unique $l_{0}^{1}\left(\mathcal{V}_{0}\right) \in \mathbb{C}$ such that:

$$
\left\{\begin{array}{l}
\Delta \mathcal{V}_{0}=0 \quad \text { in } \Omega_{N} \\
\mathcal{V}_{0}=0 \text { on } \partial \Omega_{N} \\
\lim _{r \rightarrow+\infty} \sup _{\theta \in[0,2 \pi]}\left|\mathcal{V}_{0}(r ; \theta)-\left(Y_{0,0}+\frac{2}{\pi} J_{0,0} \ln r\right)-l_{0}^{1}\left(\mathcal{V}_{0}\right)\right|=0
\end{array}\right.
$$


We call near field the function $V_{0}^{\varepsilon}(\mathbf{x})=\frac{1}{4} \mathcal{V}_{0}\left(\frac{\mathbf{x}}{\varepsilon}\right)$. It is proved in [12] that

$$
\int_{\Gamma^{\varepsilon}} \frac{\partial V_{0}^{\varepsilon}}{\partial n}=1
$$

This last property is interesting and we have chosen the multiplicative factor between $V_{0}^{\varepsilon}$ and $\mathcal{V}_{0}$ so that it is satisfied. It will be important in the following to keep in mind the expansion of $\mathcal{V}_{0}$ (and of $V_{0}^{\varepsilon}$ ) in the neighborhood of infinity. It is given by

$$
\mathcal{V}_{0}(r, \theta)=\left(Y_{0,0}+\frac{2}{\pi} J_{0,0} \ln r\right)+l_{0}^{1}\left(\mathcal{V}_{0}\right)+\sum_{|p| \geqslant 1} \frac{\mathcal{V}_{0, p}}{r|p|} e^{i p \theta} \quad, \mathcal{V}_{0, p} \in \mathbb{C} \quad \text { when } \quad r \rightarrow+\infty
$$

The sum converges in $H^{1}$ norm on any compact set sufficiently far away from the origin.

Remark: In the case where $\Omega_{\varepsilon}^{i}$ is the disk of center 0 and radius $\varepsilon$, we have

$$
V_{0}^{\varepsilon}(\mathbf{x})=\frac{1}{2 \pi} \ln \frac{|\mathbf{x}|}{\varepsilon} \mathbf{1}_{\Omega_{R} \backslash \Omega_{\varepsilon}^{i}} .
$$

\section{- The constant $\lambda_{0}$}

We now define the constant involved in the matched expansion:

$$
\lambda_{0}=\frac{\pi}{2}\left(l_{0}^{1}\left(\mathcal{V}_{0}\right)-\mathcal{L}_{0}^{1}\left(v_{0}\right)\right) .
$$

Remark: In the case where $\Omega_{\varepsilon}^{i}$ is the disk of center 0 and radius $\varepsilon$, we have

$$
\lambda_{0}=\gamma+i \frac{\pi}{2}-\ln \frac{k}{2}
$$

We invite the reader to understand formula (2.2) with the following point of view. Far from the small obstacle, more precisely for $|\mathbf{x}|>\sqrt{\varepsilon}$, the solution of the exact problem satisfies

$$
u^{\varepsilon}(\mathbf{x}) \simeq u_{0}(\mathbf{x})+\frac{-\frac{\pi}{2} u_{0}(0)}{\ln \varepsilon-\lambda_{0}} v_{0}(\mathbf{x}) .
$$

Close to the small obstacle, for $|\mathbf{x}|<\sqrt{\varepsilon} / 2$, the solution of the exact problem satisfies

$$
u^{\varepsilon}(\mathbf{x}) \simeq \frac{-2 \pi u_{0}(0)}{\ln \varepsilon-\lambda_{0}} V_{0}^{\varepsilon}(\mathbf{x})
$$

In particular, we obtain an explicit expression of the behavior of the solution on the boundary of the small obstacle. We will use later this information in order to choose a smaller space of Lagrange multipliers for the Dirichlet condition on $\Gamma^{\varepsilon}$.

We finally recall another asymptotic estimate. The result of Theorem 2.1 is an estimate in the whole domain $\Omega_{R}$. It is possible to obtain a sharper estimate away from the obstacle $\Omega_{\varepsilon}^{i}$. This is the subject of the following theorem.

\section{Theorem 2.2}

Take an open set $\Omega_{0} \subset \Omega_{R}$ such that for a given $\varepsilon$ there holds $\Omega_{0} \cap \Omega_{\varepsilon}^{i}=\emptyset$. Then, there exists $\kappa$ depending on $\Omega_{0}$ but independent of $\varepsilon$ such that

$$
\left\|u^{\varepsilon}-\widetilde{u}^{\varepsilon}\right\|_{H^{1}\left(\Omega_{0}\right)} \leqslant \kappa \varepsilon .
$$




\subsection{Simplified problem}

It is now possible to make a simple remark based on the results of the previous section,

$$
p^{\varepsilon}=\left[\frac{\partial u^{\varepsilon}}{\partial n}\right]_{\mid \Gamma^{\varepsilon}} \simeq\left[\frac{\partial \widetilde{u}^{\varepsilon}}{\partial n}\right]_{\mid \Gamma^{\varepsilon}}=\left.\frac{-2 \pi u_{0}(0)}{\ln \varepsilon-\lambda_{0}} \frac{\partial V_{0}^{\varepsilon}}{\partial n}\right|_{\Gamma^{\varepsilon}},
$$

since $\chi_{1 / 2}^{\varepsilon}=1$ on $\Gamma^{\varepsilon}$. Thus we decide to replace the space of Lagrange multipliers $Q_{\mathrm{ex}}$ by the smaller space $Q_{\text {ap }}$ defined by

$$
Q_{\text {ap }}=\left\{q=\breve{q} \frac{\partial V_{0}^{\varepsilon}}{\partial n} \quad \text { such that } \quad \breve{q} \in \mathbb{C}\right\} \subset Q_{\text {ex }}=H^{-1 / 2}\left(\Gamma^{\varepsilon}\right) .
$$

This new space has dimension one, so that it is possible to identify $q=\breve{q} \frac{\partial V_{0}^{\varepsilon}}{\partial n}$ with $\breve{q}$. So we denote $|q|$ the norm of an element of $Q_{\text {ap }}$, and this norm will be defined by $|q|=|\breve{q}|$ i.e. the modulus of $\breve{q}$ as a complex number. The problem we are interested in is then reformulated in the following manner

$$
\left\{\begin{array}{l}
\text { Find }\left(\mathfrak{u}^{\varepsilon}, \mathfrak{p}^{\varepsilon}\right) \in V \times Q_{\text {ap }} \text { such that } \\
a\left(\mathfrak{u}^{\varepsilon}, v\right)+b^{\varepsilon}\left(v, \mathfrak{p}^{\varepsilon}\right)=\langle f, v\rangle_{V, V^{\prime}} \quad \forall v \in V, \\
b^{\varepsilon}\left(\mathfrak{u}^{\varepsilon}, q\right)=0 \quad \forall q \in Q_{\mathrm{ap}} .
\end{array}\right.
$$

This problem fits in the setting of the analysis of F.Brezzi and M.Fortin in [1]. We will call it a simplified problem. In addition we consider the following notations

$$
Z^{\varepsilon}=\left\{v \in V \mid b^{\varepsilon}(v, q)=0, \forall q \in Q_{\mathrm{ap}}\right\} \quad \text { et } \quad \breve{b}^{\varepsilon}(v)=b^{\varepsilon}\left(v, \frac{\partial V_{0}^{\varepsilon}}{\partial n}\right) \quad \forall v \in V .
$$

Note in particular that $b^{\varepsilon}(v, q)=\breve{b}^{\varepsilon}(v) \breve{q}$. The linear form $\breve{b}^{\varepsilon}$ is some kind of averaging operator. Indeed when the obstacle is a disk, $\Gamma^{\varepsilon}$ is a circle of center 0 and radius $\varepsilon$, and we have $\breve{b}^{\varepsilon}(v)=$ $\frac{1}{2 \pi} \int_{0}^{2 \pi} v(\varepsilon, \theta) d \theta$.

We will prove the existence and uniqueness of the solution of the simplified problem (2.5). It is first necessary to show that an inf-sup condition is satisfied by $a$. Moreover this inf-sup condition has to be uniform with respect to $\varepsilon$ for the problem to be stable.

\section{Lemma 2.3}

There exists $\alpha_{0}>0$ independent of $\varepsilon$ such that

$$
\inf _{u \in Z^{\varepsilon}} \sup _{v \in Z^{\varepsilon}} \frac{|a(u, v)|}{\|u\|_{V}\|v\|_{V}} \geqslant \alpha_{0} .
$$

Proof: Proceed by contradiction, suppose there exists a sequence $\varepsilon_{n} \underset{n \rightarrow+\infty}{\rightarrow} 0$, and another $\left(u_{n}\right)$ such that for any $n$,

$$
u_{n} \in Z^{\varepsilon_{n}}, \quad\left\|u_{n}\right\|_{V}=1, \quad \text { et } \sup _{v \in Z^{\varepsilon_{n}}} \frac{a\left(u_{n}, v\right)}{\|v\|_{V}} \underset{n \rightarrow+\infty}{\rightarrow} 0
$$

Since $\left(u_{n}\right)$ is bounded in $H^{1}\left(\Omega_{R}\right)$, we can extract from $\left(u_{n}\right)$ a subsequence weakly converging in $H^{1}\left(\Omega_{R}\right)$ and strongly converging in $L^{2}\left(\Omega_{R}\right)$ toward, say, $u$. Take $v \in H_{00}^{1}\left(\Omega_{R} \backslash\{0\}\right)=\{w \in$ $H^{1}\left(\Omega_{R}\right)$ s.t $w=0$ on a neighborhood of 0$\}$. Then, for $n$ sufficiently large, $v \in Z^{\varepsilon_{n}}$. From the weak convergence, we obtain $a(u, v)=0$. Since $v$ is chosen arbitrarily in $H_{00}^{1}\left(\Omega_{R} \backslash\{0\}\right)$, use the fact that this space is dense in $H^{1}\left(\Omega_{R}\right)$ (simple result that is available in [3]) to see that the previous equality holds for any $v \in H^{1}\left(\Omega_{R}\right)$, which implies according to the well posedness of Helmholtz equation that $u=0$. As a consequence, $\left\|u_{n}\right\|_{L^{2}\left(\Omega_{R}\right)} \underset{n \rightarrow+\infty}{\rightarrow} 0$. Finally,

$$
\left\|\nabla u_{n}\right\|_{L^{2}\left(\Omega_{R}\right)}^{2} \leqslant\left\|\nabla u_{n}\right\|_{L^{2}\left(\Omega_{R}\right)}^{2}+\Re e\left\{\int_{\Gamma_{R}} u_{n} T_{R} u_{n}\right\}=a\left(u_{n}, u_{n}\right)+k^{2}\left\|u_{n}\right\|_{L^{2}\left(\Omega_{R}\right)}^{2} .
$$


Since $a\left(u_{n}, u_{n}\right) \underset{n \rightarrow+\infty}{\rightarrow} 0$, this leads to $\left\|u_{n}\right\|_{H^{1}\left(\Omega_{R}\right)} \underset{n \rightarrow+\infty}{\rightarrow} 0$. This contradicts our initial assumption, so the result of the lemma is proved.

In addition we also have to prove that an Inf-Sup condition is satisfied by $b^{\varepsilon}$. For stability sake, this condition has also to be uniformly satisfied with respect to $\varepsilon$.

\section{Lemma 2.4}

There exists $\beta_{0}>0$ independent of $\varepsilon$ such that

$$
\sup _{v \in V} \frac{\left|b^{\varepsilon}(v, q)\right|}{\|v\|_{V}} \geqslant \beta_{0}|q| \quad \forall q \in Q .
$$

\section{Proof:}

It is sufficient to consider a fixed function $u \in C^{\infty}\left(\Omega_{R}\right)$ taking the value 1 on a neighborhood of the origin. Thus, there holds

$$
\sup _{v \in V} \frac{\left|b^{\varepsilon}(v, q)\right|}{\|v\|_{V}} \geqslant \frac{|q|}{\|u\|_{V}}
$$

Since $u$ is fixed an does not depend on $\varepsilon$, this provides the desired result.

Finally, we need to prove continuity properties, uniform with respect to $\varepsilon$. this will allow us to apply classical results of existence and uniqueness for saddle-point problems.

\section{Lemma 2.5}

There exists $\kappa>0$ independent of $\varepsilon$ such that

$$
\begin{array}{lll}
\text { (i) } & |a(u, v)| \leqslant \kappa\|u\|_{V}\|v\|_{V} & \forall u, v \in V, \\
\text { (ii) } & \left|b^{\varepsilon}(v, q)\right| \leqslant \kappa \sqrt{\ln 1 / \varepsilon}\|v\|_{V}|q| & \forall(v, q) \in V \times Q_{\mathrm{ap}} .
\end{array}
$$

\section{Proof:}

Inequality $(i)$ about $a$ is trivial. Let us concentrate on (ii). Using cut-off technique, it is sufficient to prove this property for $v \in \mathcal{D}\left(\Omega_{R}\right)$. Using Lemma A.1 in the appendix, we know that there exists $\kappa>0$ independent of $\varepsilon$ such that

$$
\left|\int_{\Gamma^{\varepsilon}} \frac{\partial V_{0}^{\varepsilon}}{\partial n} \bar{v}\right| \leqslant \kappa \int_{\theta=0}^{2 \pi}|v(\varepsilon \gamma(\theta), \theta)| d \theta .
$$

Moreover,

$$
|v(\varepsilon \gamma(\theta), \theta)|=\left|\int_{R}^{\varepsilon \gamma(\theta)} \frac{\partial v}{\partial r}(r, \theta) d r\right| \leqslant\left(\int_{\varepsilon \gamma(\theta)}^{R} \frac{d r}{r}\right)^{1 / 2}\left(\int_{\varepsilon \gamma(\theta)}^{R}\left|\frac{\partial v}{\partial r}\right|^{2} r d r\right)^{1 / 2}
$$

hence

$$
\begin{aligned}
\int_{0}^{2 \pi}|v(\varepsilon \gamma(\theta), \theta)| d \theta & \leqslant\left(\int_{0}^{2 \pi} \int_{\varepsilon \gamma(\theta)}^{R} \frac{1}{r} d r d \theta\right)^{1 / 2}\left(\int_{0}^{2 \pi} \int_{\varepsilon \gamma(\theta)}^{R}\left|\frac{\partial v}{\partial r}\right|^{2} r d r d \theta\right)^{1 / 2} \\
& \leqslant \kappa \sqrt{\ln 1 / \varepsilon}\|v\|_{H^{1}\left(\Omega_{R}\right)},
\end{aligned}
$$

which yields $(i i)$.

With Lemmas 2.3, 2.4 and 2.5] we can apply Theorem $1.1 \S$ II.1 of [1] which provides existence, uniqueness and stability of the solution of problem (2.5). This can be written in the following manner, 
For any $f \in V^{\prime}$, there exists a unique couple $\left(\mathfrak{u}^{\varepsilon}, \mathfrak{p}^{\varepsilon}\right) \in V \times Q_{\text {ap }}$ such that,

$$
\begin{aligned}
& a\left(\mathfrak{u}^{\varepsilon}, v\right)+b^{\varepsilon}\left(v, \mathfrak{p}^{\varepsilon}\right)=\langle f, v\rangle_{V, V^{\prime}} \quad \forall v \in V, \\
& b^{\varepsilon}\left(\mathfrak{u}^{\varepsilon}, q\right)=0 \quad \forall q \in Q_{\mathrm{ap}} .
\end{aligned}
$$

Moreover the following estimates (uniform with respect to $\varepsilon$ ) hold

$$
\left\|\mathfrak{u}^{\varepsilon}\right\|_{V} \leqslant \frac{1}{\alpha_{0}}\|f\|_{V^{\prime}} \quad \text { et } \quad\left|\mathfrak{p}^{\varepsilon}\right| \leqslant \frac{1}{\beta_{0}}\left(1+\frac{\|a\|}{\alpha_{0}}\right)\|f\|_{V^{\prime}} .
$$

It is possible to provide an asymptotic analysis for $\mathfrak{u}^{\varepsilon}$, similar to the one for $u^{\varepsilon}$, in order to determine its behavior when $\varepsilon$ gets very small.

\section{Theorem 2.3}

There exists $\kappa>0$ independent of $\varepsilon$ such that for $\varepsilon$ small enough

$$
\left\|\widetilde{u}^{\varepsilon}-\mathfrak{u}^{\varepsilon}\right\|_{H^{1}\left(\Omega_{R}\right)} \leqslant \kappa \varepsilon^{1 / 2} .
$$

This result is similar to Theorem 2.1] and its proof follows the same lines. However, for the construction of the matched expansion of $\mathfrak{u}^{\varepsilon}$, the near field has to be characterized as the solution of a simplified problem. Theorem 2.3 yields that the first terms of the matched expansion of $\mathfrak{u}^{\varepsilon}$ coincide with the first terms of the expansion of $u^{\varepsilon}$ (but these two expansions do not coincide for higher terms). Anew, Theorem 2.3 provides a global estimate (on $\Omega_{R}$ ) but it is possible to look for some local estimate away from the obstacle. Here is a result similar to Theorem 2.2

\section{Theorem 2.4}

Take an open set $\Omega_{0} \subset \Omega_{R}$ such that for a given $\varepsilon$ there holds $\Omega_{0} \cap \Omega_{\varepsilon}^{i}=\emptyset$. Then, there exists $\kappa$ depending on $\Omega_{0}$ but independent of $\varepsilon$ such that,

$$
\left\|\mathfrak{u}^{\varepsilon}-\widetilde{u}^{\varepsilon}\right\|_{H^{1}\left(\Omega_{0}\right)} \leqslant \kappa \varepsilon \text {. }
$$

\section{Discretization of the simplified problem}

We will apply standard finite element approximation to Problem (2.5). In the rest of this paper $h$ will refer to the step of the mesh, and will tend to 0 . Consider $\left(\mathcal{T}_{h}\right)_{h>0}$, some triangulation of $\Omega_{R}$. This triangulation can be composed of triangles or quadrangles. For any $h$, take $\left(K, \Sigma_{K}, P_{K}\right)_{K \in \mathcal{T}_{h}}$ a collection of finite elements of order $q$ associated with $\mathcal{T}_{h}$. Let $V^{h}=\left\{u \in C^{0}\left(\Omega_{R}\right) \mid u_{\mid K} \in\right.$ $\left.P_{K}\right\}$. We denote $I_{h}$ the number of degrees of freedom, $\left(a_{i}^{h}\right)_{i=1 \ldots I}$ these degrees of freedom, and $\left(\varphi_{i}^{h}\right)_{i=1 \ldots I_{h}}$ the associated basis of shape functions $\left(\varphi_{i}^{h}\right.$ is associated to $\left.a_{i}\right)$. For any $K \in \mathcal{T}_{h}$, denote $\rho_{K}$, the maximal radius of circles contained in $K$, and denote $h_{K}=\operatorname{diam}(K)$. We define $\rho(h)=$ $\min _{K \in \mathcal{T}_{h}} \rho_{K}$ and $h=\max _{K \in \mathcal{T}_{h}} h_{K}$. We will make the following assumption on the triangulation $\mathcal{T}_{h}$

$$
\text { H1: There exists } \sigma_{+}, \sigma_{-}>0 \text { such that for any } h>0, \sigma_{-}<\frac{\rho(h)}{h}<\sigma_{+} \text {. }
$$

We say that the triangulation $\mathcal{T}_{h}$ is regular. This means that it does not contain any mesh refinement, in particular not in the neighborhood of the obstacle. This assumption is verified in the applications we are interested in. In the discretized problem that we want to solve, the solution denoted $u^{\varepsilon, h}$ depends on two small parameters: $\varepsilon$ and $h$. A natural question is whether or not $u^{\varepsilon, h}$ is a good approximation of $u^{\varepsilon}$ when $\varepsilon^{2}+h^{2} \rightarrow 0$. However, for technical reasons, we cannot suppose that $\varepsilon$ and $h$ go to 0 completely independently. As a consequence we will look at $\varepsilon$ as a function of $h, \varepsilon=\varepsilon(h)$. We will suppose that the mesh is coarse in comparison with the diameter of the obstacle, and then make the following assumption 
H2: There exists $\kappa>0$ independent of $h$, such that $\varepsilon(h) \leqslant \kappa h$.

This corresponds to a zone in the plane $(h, \varepsilon)$ similar to the region that is not colored in gray in Figure 2

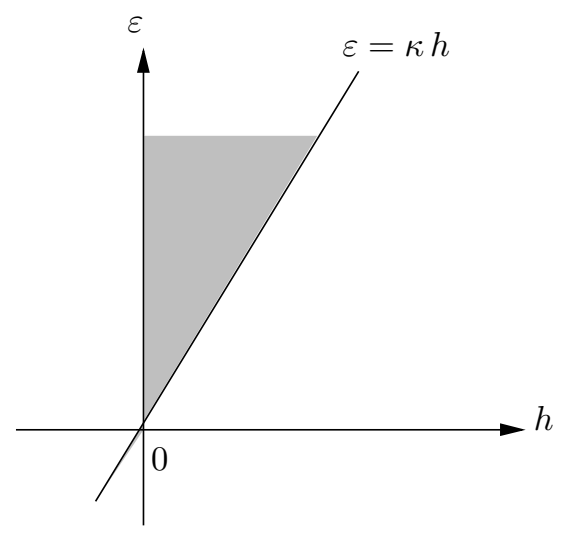

Figure 2: Coarse mesh assumption

We denote $V^{h}$ the standard Galerkin approximation space corresponding to this discretization. Remind that $q$ is the order of the elements.

$$
V^{h}=\left\{v \in C^{0}\left(\Omega_{R}\right) \text { s.t } \forall K \in \mathcal{T}_{h}, v_{\mid K} \in \mathbb{P}_{q}\left(\text { or } \mathbb{Q}_{q}\right)\right\}
$$

$V^{h}=\operatorname{span}\left(\varphi_{i}^{h}\right)_{i=1 \ldots I_{h}}$. Given a function $v \in C^{0}\left(\Omega_{R}\right)$, we denote $\Pi_{h} v$ its interpolant constructed with usual shape functions, more precisely

$$
\Pi_{h} v=\sum_{i=1}^{I} v\left(a_{i}\right) \varphi_{i}^{h} .
$$

For simplicity sake, we will sometimes write $\varphi_{i}, I$ and $a_{i}$ instead of $\varphi_{i}^{h}, I_{h}$ and $a_{i}^{h}$.

\subsection{Solution of the simplified problem using standard discretization}

\subsubsection{Formulation}

We want to solve the simplified problem (2.5). If we apply a discretization corresponding to the assumptions we formulated previously, we are led to the following discrete formulation :

$$
\left\{\begin{array}{l}
\text { Find } \quad\left(u^{\varepsilon, h}, p^{\varepsilon, h}\right) \in V^{h} \times Q_{h} \quad \text { such that, } \\
a\left(u^{\varepsilon, h}, w_{h}\right)+b^{\varepsilon}\left(w_{h}, p^{\varepsilon, h}\right)=\left\langle f, w_{h}\right\rangle_{V, V^{\prime}} \quad \forall w_{h} \in V^{h}, \\
b^{\varepsilon}\left(u^{\varepsilon, h}, q_{h}\right)=0 . \quad \forall q_{h} \in Q_{h}
\end{array}\right.
$$

Again, for this problem to be well posed and for $u^{\varepsilon, h}$ to be a good approximation of $\mathfrak{u}^{\varepsilon}$, it is necessary that $a$ and $b^{\varepsilon}$ restricted to $V^{h} \times Q^{h}$ verify inf-sup conditions and continuity conditions, uniform with respect to $\varepsilon$ and $h$. The continuity conditions are verified in the same manner as for the continuous problems. We will deal with inf-sup conditions with the two next lemmas.

\subsubsection{Stability study}

We define $Z^{\varepsilon, h}=\left\{w_{h} \in V^{h} \mid b^{\varepsilon}\left(w_{h}, q_{h}\right)=0 \forall q_{h} \in Q_{h}\right\}=\left\{w_{h} \in V^{h} \mid \int_{\Gamma^{\varepsilon}} \frac{\partial V_{0}^{\varepsilon}}{\partial n} w_{h}=0\right\}$. It is an intermediate space that we need to introduce in order to formulate the next lemma and the associated inf-sup condition. 


\section{Lemma 3.1}

There exists $\kappa>0$ independent of $\varepsilon$ and $h$ such that

$$
\inf _{u_{h} \in Z^{\varepsilon, h}} \sup _{v_{h} \in Z^{\varepsilon, h}} \frac{\left|a\left(u_{h}, v_{h}\right)\right|}{\left\|u_{h}\right\|_{V}\left\|v_{h}\right\|_{V}} \geqslant \kappa .
$$

\section{Proof:}

Proceed by contradiction and suppose that there exists two sequences $\left(\varepsilon_{n}\right)$ and $\left(h_{n}\right)$ such that $\varepsilon_{n}+h_{n} \underset{n \rightarrow+\infty}{\rightarrow} 0$, and a sequence $\left(u_{n}\right)$ such that for any $n$,

$$
u_{n} \in Z^{\varepsilon_{n}, h_{n}}, \quad\left\|u_{n}\right\|_{V}=1 \quad \text { and } \sup _{v_{h} \in Z_{h_{n}}^{\varepsilon_{n}}} \frac{\left|a\left(u_{n}, v_{h}\right)\right|}{\left\|v_{h}\right\|_{V}} \underset{n \rightarrow+\infty}{\rightarrow} 0
$$

Since $\left(u_{n}\right)$ is bounded in $H^{1}\left(\Omega_{R}\right)$, it is possible to extract a subsequence weakly converging in $H^{1}\left(\Omega_{R}\right)$ and strongly converging in $L^{2}\left(\Omega_{R}\right)$ toward $u \in H^{1}\left(\Omega_{R}\right)$. Take $v \in H_{00}^{1}\left(\Omega_{R} \backslash\{0\}\right) \cap$ $C^{\infty}\left(\Omega_{R}\right)$; then, for $n$ large enough, $\Pi_{h_{n}} v \in Z^{\varepsilon_{n}, h_{n}}$.

$$
a\left(u_{n}, v\right)=a\left(u_{n}, \Pi_{h_{n}} v\right)+a\left(u_{n}, v-\Pi_{h_{n}} v\right) \underset{n \rightarrow+\infty}{\rightarrow} 0 \quad \text { and } \quad a\left(u_{n}, v\right) \underset{n \rightarrow+\infty}{\rightarrow} a(u, v) .
$$

Since $v$ is chosen arbitrarily in $H_{00}^{1}\left(\Omega_{R} \backslash\{0\}\right)$, this space being dense in $H^{1}\left(\Omega_{R}\right)$, we conclude that $a(u, v)=0, \forall v \in H^{1}\left(\Omega_{R}\right)$. It is classical that this last property implies $u=0$. Thus $\left\|u_{n}\right\|_{L^{2}\left(\Omega_{R}\right)} \underset{n \rightarrow+\infty}{\rightarrow} 0$. Finally,

$$
\left\|\nabla u_{n}\right\|_{L^{2}\left(\Omega_{R}\right)}^{2} \leqslant\left\|\nabla u_{n}\right\|_{L^{2}\left(\Omega_{R}\right)}^{2}+\Re e\left\{\int_{\Gamma_{R}} u_{n} T_{R} u_{n}\right\}=a\left(u_{n}, u_{n}\right)+k^{2}\left\|u_{n}\right\|_{L^{2}\left(\Omega_{R}\right)}^{2} .
$$

Since $a\left(u_{n}, u_{n}\right) \underset{n \rightarrow+\infty}{\rightarrow} 0$, we obtain that $\left\|u_{n}\right\|_{H^{1}\left(\Omega_{R}\right)} \underset{n \rightarrow+\infty}{\rightarrow} 0$. This leads to a contradiction which yields the desired result.

Now we also have to prove an inf-sup condition on $b^{\varepsilon}$ uniform with respect to $\varepsilon$ and $h$.

\section{Lemma 3.2}

There exists $\kappa>0$ independent of $h$ and $\varepsilon$ such that

$$
\forall q_{h} \in Q_{h}, \quad \sup _{w_{h} \in V^{h}} \frac{\left|b^{\varepsilon}\left(w_{h}, q_{h}\right)\right|}{\left\|w_{h}\right\|_{V}} \geqslant \kappa\left|q_{h}\right|,
$$

\section{Proof:}

Consider a fixed function $v \in C^{\infty}$ with compact support included in $\Omega_{R}$ and taking the value 1 on a neighborhood of the origin 0 . Take its Lagrange interpolant $\Pi_{h} v$. There holds $\left\|\Pi_{h} v-v\right\|_{H^{1}\left(\Omega_{R}\right)} \leqslant \kappa h\|v\|_{H^{2}\left(\Omega_{R}\right)}$, where $\kappa$ is constant, depending only on the geometry of $\Omega_{R}$ (and so independent of $\varepsilon$ ). Then there exists $h_{0}>0$ independent of $\varepsilon$ such that for any $h \in$ ] $0, h_{0}\left[,\left\|\Pi_{h} v\right\|_{H^{1}\left(\Omega_{R}\right)} \leqslant 2\|v\|_{H^{1}\left(\Omega_{R}\right)}\right.$, and $\Pi_{h} v=v=1$ on a neighborhood of the origin 0 . Moreover for $\varepsilon>0$ small enough, $\int_{\Gamma^{\varepsilon}} \frac{\partial V_{0}^{\varepsilon}}{\partial n}=1$, so that for any $q_{h} \in \mathbb{C}$, if we choose $w_{h}=\Pi_{h} v \exp \left(-i \arg \left(q_{h}\right)\right)$,

$$
\sup _{w_{h} \in V^{h}} \frac{q_{h} \int_{\Gamma^{\varepsilon}} \frac{\partial V_{0}^{\varepsilon}}{\partial n} w_{h}}{\left\|w_{h}\right\|_{H^{1}\left(\Omega_{R}\right)}} \geqslant \frac{\left|q_{h}\right| \int_{\Gamma^{\varepsilon}} \frac{\partial V_{0}^{\varepsilon}}{\partial n} \Pi_{h} v}{\left\|\Pi_{h} v\right\|_{H^{1}\left(\Omega_{R}\right)}}=\frac{\left|q_{h}\right|}{\left\|\Pi_{h} v\right\|_{H^{1}\left(\Omega_{R}\right)}} \geqslant \frac{\left|q_{h}\right|}{2\|v\|_{H^{1}\left(\Omega_{R}\right)}},
$$


Since $v$ is fixed and thus independent of $h$ and $\varepsilon$, this proves the desired result.

Now, according to Lemmas 3.13 .2 and 2.5] we can apply propositions 2.4 et 2.5 §II.2 of [1] that give us existence, uniqueness and stability of the solution of problem (3.1). We obtain

For any $f \in V^{\prime}$, there exists a unique couple $\left(u^{\varepsilon, h}, p^{\varepsilon, h}\right) \in V^{h} \times Q_{h}$ such that

$$
\begin{aligned}
& a\left(u^{\varepsilon, h}, w_{h}\right)+b^{\varepsilon}\left(w_{h}, p^{\varepsilon, h}\right)=\left\langle f, w_{h}\right\rangle_{V, V^{\prime}} \quad \forall w_{h} \in V^{h} \\
& b^{\varepsilon}\left(u^{\varepsilon, h}, q_{h}\right)=0 \quad \forall q_{h} \in Q_{h} .
\end{aligned}
$$

Moreover the following estimates (uniform with respect to $\varepsilon$ ) hold

$$
\begin{aligned}
& \left\|u^{\varepsilon, h}-\mathfrak{u}^{\varepsilon}\right\|_{V} \leqslant\left(1+\frac{\|a\|}{\alpha_{0}}\right)\left(1+\frac{\left\|b^{\varepsilon}\right\|}{\beta_{0}}\right) \inf _{w_{h} \in V^{h}}\left\|\mathfrak{u}^{\varepsilon}-w_{h}\right\|_{V} \leqslant \kappa \sqrt{\ln 1 / \varepsilon} \inf _{w_{h} \in V^{h}}\left\|\mathfrak{u}^{\varepsilon}-w_{h}\right\|_{V} . \\
& \left\|p^{\varepsilon, h}-\mathfrak{p}^{\varepsilon}\right\|_{V} \leqslant \frac{\|a\|}{\beta_{0}}\left\|u^{\varepsilon, h}-\mathfrak{u}^{\varepsilon}\right\|_{V} \leqslant \kappa \sqrt{\ln 1 / \varepsilon} \inf _{w_{h} \in V^{h}}\left\|\mathfrak{u}^{\varepsilon}-w_{h}\right\|_{V} .
\end{aligned}
$$

According to this last result, the numerical scheme (3.1) has good stability properties so that it is sufficient that $\inf _{w_{h} \in V^{h}}\left\|\mathfrak{u}^{\varepsilon}-w_{h}\right\|_{H^{1}\left(\Omega_{R}\right)}$ be small for $u^{\varepsilon, h}$ to be a good approximation of $\mathfrak{u}^{\varepsilon}$. The problem is that this quantity is not small! It is possible to understand it in the following manner. When $\varepsilon$ goes to $0, \mathfrak{u}^{\varepsilon}$ becomes logarithmically singular in the neighborhood of the small obstacle $\Omega_{\varepsilon}^{i}$, and since $\mathbf{x} \mapsto \ln |\mathbf{x}|$ does not belong to $H^{1}\left(\Omega_{R}\right)$, the space $V^{h}$ has big difficulties in approximating $\mathfrak{u}^{\varepsilon}$. It is also possible to understand this situation in the following manner. Usually we use classical consistancy estimates in order to obtain,

$$
\inf _{w_{h} \in V^{h}}\left\|\mathfrak{u}^{\varepsilon}-w_{h}\right\|_{H^{1}\left(\Omega_{R}\right)} \leqslant \kappa\left\|\mathfrak{u}^{\varepsilon}\right\|_{H^{s}\left(\Omega_{R}\right)} h^{s-1},
$$

but we can prove for particular cases that $\left\|\mathfrak{u}^{\varepsilon}\right\|_{H^{s}\left(\Omega_{R}\right)} \sim \kappa / \varepsilon^{s-1}$ when $\varepsilon \rightarrow 0$. Hence, for a given $s$, we can only hope for bounding $\left\|\mathfrak{u}^{\varepsilon}-u^{\varepsilon, h}\right\|_{H^{1}\left(\Omega_{R}\right)}$ with a quantity proportional to $h^{s} / \varepsilon^{s} \geqslant \kappa>0$ according to assumption H2. In a sense, there is no consistency! We will now illustrate this with a numerical experiment.

\subsection{Numerical experiments for the simplified scheme}

For these experiments, we have assumed that $\Omega_{R}$ is a disk of center 0 and radius $R$ (there is no obstacle but a small one $\Omega_{\varepsilon}^{i}$ ). For the small obstacle, we have considered a disk of center 0 and radius $\varepsilon$,

$$
\left(\Gamma^{\varepsilon}\right): r=\varepsilon .
$$

We have considered an incident plane wave $u_{i n c}(\mathbf{x})=-e^{i k e_{x} \cdot \mathbf{x}}$ where $e_{x}$ refers to the $x$ direction of the Cartesian coordinates in $\mathbb{R}^{2}$. The Jacobi-Anger formula yields a Fourier decomposition of $u_{i n c}$ and in particular its trace on the wire $\Gamma^{\varepsilon}$

$$
u_{i n c \mid \Gamma^{\varepsilon}}(\mathbf{x})=-J_{0}(k \varepsilon)-\sum_{|p| \geqslant 1} i^{|p|} J_{|p|}(k \varepsilon) e^{i p \theta} .
$$


In this situation we have an analytical expression for $u^{\varepsilon}$ and $\mathfrak{u}^{\varepsilon}$,

$$
\begin{aligned}
& u^{\varepsilon}(\mathbf{x})-u_{i n c}(\mathbf{x})= \begin{cases}\frac{J_{0}(k \varepsilon)}{H_{0}^{(1)}(k \varepsilon)} H_{0}^{(1)}(k|\mathbf{x}|)+\sum_{|p| \geqslant 1} i^{|p|} \frac{J_{|p|}(k \varepsilon)}{H_{|p|}^{(1)}(k \varepsilon)} H_{|p|}^{(1)}(k|\mathbf{x}|) e^{i p \theta} & \text { if } \quad|\mathbf{x}| \geqslant \varepsilon \\
J_{0}(k|\mathbf{x}|)+\sum_{|p| \geqslant 1} i^{|p|} J_{|p|}(k|\mathbf{x}|) e^{i p \theta} & \text { if } \quad|\mathbf{x}| \leqslant \varepsilon .\end{cases} \\
& \mathfrak{u}^{\varepsilon}(\mathbf{x})= \begin{cases}\frac{J_{0}(k \varepsilon)}{H_{0}^{(1)}(k \varepsilon)} H_{0}^{(1)}(k|\mathbf{x}|) & \text { if }|\mathbf{x}| \geqslant \varepsilon \\
J_{0}(k|\mathbf{x}|) & \text { if }|\mathbf{x}| \leqslant \varepsilon .\end{cases}
\end{aligned}
$$

Denote $\mathfrak{p}^{\varepsilon}$, the jump of the normal derivative of $\mathfrak{u}^{\varepsilon}$. Then, $\left(\mathfrak{u}^{\varepsilon}, \mathfrak{p}^{\varepsilon}\right)$ is the unique couple satisfying

$$
\left\{\begin{array}{l}
\left(\mathfrak{u}^{\varepsilon}, \mathfrak{p}^{\varepsilon}\right) \in H^{1}\left(\Omega_{R}\right) \times \mathbb{C} \quad \text { such that, } \\
\int_{\Omega_{R}} \nabla \mathfrak{u}^{\varepsilon} \cdot \nabla \bar{v}-k^{2} \int_{\Omega_{R}} \mathfrak{u}^{\varepsilon} \bar{v}+\lambda_{R} \int_{\Gamma_{R}} \bar{v} \mathfrak{u}^{\varepsilon}+\int_{\Gamma^{\varepsilon}} \mathfrak{p}^{\varepsilon} \bar{v}=\int_{\Omega_{R}} f \bar{v} \quad \forall v \in H^{1}\left(\Omega_{R}\right), \\
\int_{\Gamma^{\varepsilon}} q \mathfrak{u}^{\varepsilon}=0 \quad \forall q \in \mathbb{C} .
\end{array}\right.
$$

In this formulation, the term on the exterior boundary stands for an order one condition with impedance given by

$$
\lambda_{R}=-\frac{H_{0}^{(1)^{\prime}}(k R)}{H_{0}^{(1)}(k R)} .
$$

We numerically solved problem (3.2). using the code MONTJOIE developed by famous Marc Durufle. It is detailed in his $\mathrm{PhD}$ thesis [6], and a complete documentation is available on http://wwwrocq.inria.fr/ durufle/montjoie.html . We carried out these experiments with several orders of finite elements. Below is represented the results for order 3 quadrangular finite elements. The wavelength is $\lambda=1$, and so $k=2 \pi / \lambda=2 \pi$. What is represented in Figure 3 is the relative error in $H^{1}$ norm in the zone $\Omega_{R} \backslash \overline{D\left(0, R^{\prime}\right)}=\left\{\mathbf{x} \in \Omega_{R}\right.$ t.q $\left.1<|\mathbf{x}|<3\right\}$, away from the small obstacle

$$
\text { Error }=\frac{\left\|\mathfrak{u}^{\varepsilon}-u^{\varepsilon, h}\right\|_{H^{1}\left(\Omega_{R} \backslash \overline{\Omega_{R^{\prime}}}\right)}}{\left\|\mathfrak{u}^{\varepsilon}\right\|_{H^{1}\left(\Omega_{R} \backslash \overline{\Omega_{R^{\prime}}}\right)}} .
$$

Of course, this error does not take into account the error of the model; that is to say $\| u^{\varepsilon}-$ $\mathfrak{u}^{\varepsilon} \|_{H^{1}\left(\Omega_{R} \backslash \Omega_{R^{\prime}}\right)}$. 


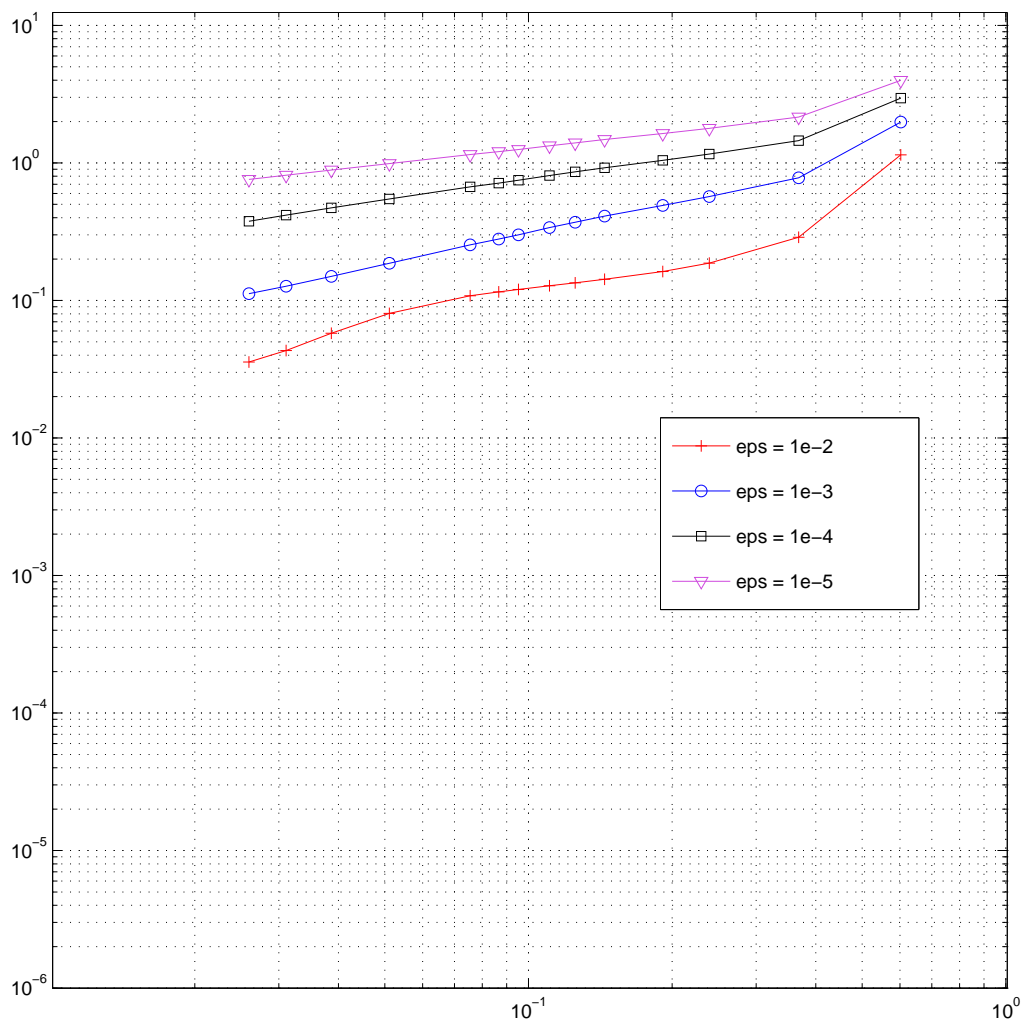

Figure 3: Relative error versus step of the mesh

The results of figure 3 are not very good since for $\varepsilon / \lambda=0.01$ and $h / \lambda$ with about the same value (100 points per wavelength), we obtain a relative error greater than $10^{-2}$, which is not very sharp. Moreover for $\varepsilon / \lambda$ a lot smaller, for exemple $\varepsilon / \lambda=10^{-5}$, the relative error is approximately equal to $1\left(100 \%\right.$ !) even for $h / \lambda=10^{-2}$. The table below yields the rates of convergence for this graphs. We can see that these rates are not better than 1 whereas we used order 3 finite elements, and the situation becomes worse when $\varepsilon \rightarrow 0$.

\begin{tabular}{|c|c|}
\hline$\varepsilon / \lambda$ & rate \\
\hline $10^{-2}$ & 0.9051 \\
\hline $10^{-3}$ & 0.8697 \\
\hline $10^{-4}$ & 0.6163 \\
\hline $10^{-5}$ & 0.5074 \\
\hline
\end{tabular}

In the particular case we considered here, we can also analytically compute $\mathfrak{p}^{\varepsilon}$. Indeed using the analytical expression of $\mathfrak{u}^{\varepsilon}$ and the Wronskian formula for Bessel functions (that can be found in [10] for exemple), we obtain

$$
\begin{aligned}
\mathfrak{p}^{\varepsilon} & =\left[\frac{\partial \mathfrak{u}^{\varepsilon}}{\partial n}\right]_{\Gamma^{\varepsilon}}=k \frac{J_{0}(k \varepsilon) H_{0}^{(1)^{\prime}}(k \varepsilon)-J_{0}^{\prime}(k \varepsilon) H_{0}^{(1)}(k \varepsilon)}{H_{0}^{(1)}(k \varepsilon)} \\
& =i k \frac{J_{0}(k \varepsilon) Y_{0}^{\prime}(k \varepsilon)-J_{0}^{\prime}(k \varepsilon) Y_{0}(k \varepsilon)}{H_{0}^{(1)}(k \varepsilon)}=k \frac{2 i}{\pi k \varepsilon} \frac{1}{H_{0}^{(1)}(k \varepsilon)}=\frac{2 i}{\pi \varepsilon H_{0}^{(1)}(k \varepsilon)} .
\end{aligned}
$$


Hence, $\breve{\mathfrak{p}}^{\varepsilon}=2 \pi \varepsilon \mathfrak{p}^{\varepsilon}=\frac{4 i}{H_{0}^{(1)}(k \varepsilon)}$. Thus, we can take as a relative error the quantity

$$
\text { Error }=\frac{\left|\breve{\mathfrak{p}}^{\varepsilon}-\breve{p}^{\varepsilon, h}\right|}{\left|\breve{\mathfrak{p}}^{\varepsilon}\right|}
$$

We obtained the same graphs as previously but for $\breve{\mathfrak{p}}^{\varepsilon}$. The results are represented in Figure 4
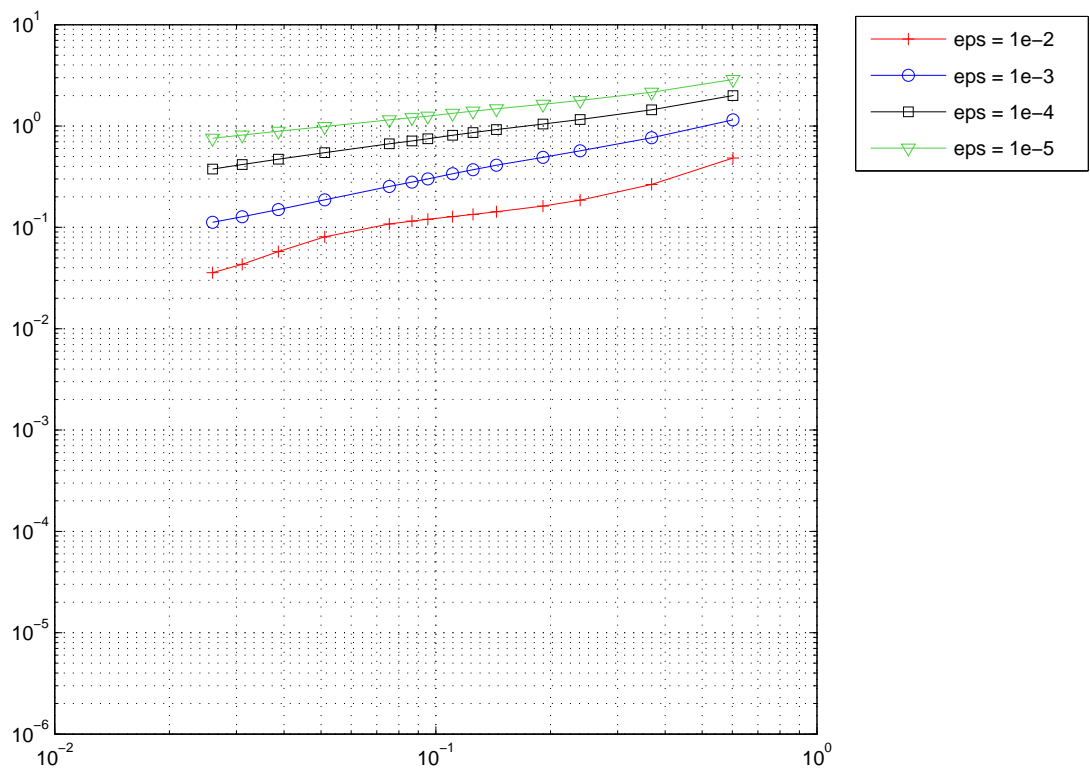

Figure 4: Relative error for the Lagrange multipliers versus step of the mesh

The graphs of figure 4 are qualitatively and nearly quantitatively identical to the one of Figure 3 In the following table we present the rates of convergence that we obtained

\begin{tabular}{|c|c|}
\hline$\varepsilon / \lambda$ & rate \\
\hline $10^{-2}$ & 0.7373 \\
\hline $10^{-3}$ & 0.7352 \\
\hline $10^{-4}$ & 0.5139 \\
\hline $10^{-5}$ & 0.4067 \\
\hline
\end{tabular}

\section{Augmented Galerkin scheme}

\subsection{Presentation and first analysis of the method}

We will remedy the lack of consistency that we put in evidence in the previous section. As we pointed out before, it comes from the logarithmic behavior of the exact solution when $\varepsilon \rightarrow 0$ that the standard approximation space cannot correctly reproduce. We will then augment the approximation space with an additionnal shape function whose role will be to reproduce the singular behavior appearing in the asymptotic expansion. The additional shape function $\psi^{\varepsilon} \in$ $H^{1}\left(\Omega_{R}\right)$ will be no other than the near field (see section 2.1) truncated by a fixed cut-off function.

$$
\psi^{\varepsilon}(\mathbf{x})=2 \pi \chi_{0}(\mathbf{x}) V_{0}^{\varepsilon}(\mathbf{x}) \mathbf{1}_{\Omega_{R} \backslash \overline{\Omega_{\varepsilon}^{i}}}
$$


We define the augmented approximation space by

$$
V_{\mathbf{e}}^{h}=\left\{u \in C^{0}\left(\Omega_{R}\right) \mid \exists(v, \alpha) \in V^{h} \times \mathbb{C} \text { s.t } u=v+\alpha \psi^{\varepsilon},\right\}
$$

and also define $Z_{\mathbf{e}}^{\varepsilon, h}=\left\{w_{h} \in V_{\mathbf{e}}^{h} \mid b^{\varepsilon}\left(w_{h}, q_{h}\right)=0 \forall q_{h} \in Q_{h}\right\}=\left\{w_{h} \in V_{\mathbf{e}}^{h} \mid \int_{\Gamma^{\varepsilon}} \frac{\partial V_{0}^{\varepsilon}}{\partial n} w_{h}=0.\right\}$.

Remark: In the case where $\Omega_{\varepsilon}^{i}$ is a disk of center 0 and radius $\varepsilon$, the expression of the additional shape function is greatly simplified. Indeed there holds

$$
\psi^{\varepsilon}(\mathbf{x})=\chi_{0}(\mathbf{x}) \mathbf{1}_{\Omega_{R} \backslash \overline{\Omega_{\varepsilon}^{i}}}(\mathbf{x}) \frac{1}{2 \pi} \ln \frac{|\mathbf{x}|}{\varepsilon} .
$$

Now we are looking for $u^{\varepsilon, h}$ solution to the discrete simplified problem posed with this new approximation space. The formulation of the discrete problem is now

$$
\left\{\begin{array}{l}
\text { Find } \quad\left(u^{\varepsilon, h}, p^{\varepsilon, h}\right) \in V_{\mathbf{e}}^{h} \times Q_{h} \quad \text { such that } \\
a\left(u^{\varepsilon, h}, w_{h}\right)+b^{\varepsilon}\left(w_{h}, p^{\varepsilon, h}\right)=\left\langle f, w_{h}\right\rangle_{V, V^{\prime}} \quad \forall w_{h} \in V_{\mathbf{e}}^{h} \\
b^{\varepsilon}\left(u^{\varepsilon, h}, q_{h}\right)=0 \quad \forall q_{h} \in Q_{h} .
\end{array}\right.
$$

The stability study of the previous section is still valid in this case. Note that since $\psi^{\varepsilon}=0$ on $\Gamma^{\varepsilon}$, the results of Lemmas 3.1 and 3.2 still hold replacing $V^{h}$ by $V_{\mathbf{e}}^{h}$. We can thus prove existence and uniqueness for the solution of problem (4.1), and also obtain the estimate

$$
\left\|\mathfrak{u}^{\varepsilon}-u^{\varepsilon, h}\right\|_{H^{1}\left(\Omega_{R}\right)} \leqslant \kappa \sqrt{\ln 1 / \varepsilon} \inf _{w_{h} \in V_{\mathbf{e}}^{h}}\left\|\mathfrak{u}^{\varepsilon}-w_{h}\right\|_{H^{1}\left(\Omega_{R}\right)},
$$

for a suitable $\kappa>0$ independent of $\varepsilon$. This time, the right-hand side of this inequality has some chance to be small since the approximation space $V_{\mathbf{e}}^{h}$ contains $\psi^{\varepsilon}$.

\subsection{Global consistency}

Now, we present a first result of global consistency that gives a bound for the error on the whole domain $\Omega_{R}$. The proof directly uses the asymptotic analysis for which we recalled some results in Paragraph 2.1.

\section{Theorem 4.1}

There exists $\kappa>0$ independent of $\varepsilon$ and $h$ such that $\inf _{w_{h} \in V_{\mathrm{e}}^{h}}\left\|\mathfrak{u}^{\varepsilon}-w_{h}\right\|_{H^{1}\left(\Omega_{R}\right)} \leqslant \kappa\left(\varepsilon^{1 / 2}+h\right)$.

\section{Proof:}

In order to estimate the left-hand side of the above inequality we will use the asymptotic expansion that we previously introduced. Let us apply Theorem 2.3

$$
\left\|\mathfrak{u}^{\varepsilon}-\widetilde{u}^{\varepsilon}\right\|_{H^{1}\left(\Omega_{R}\right)} \leqslant \kappa \varepsilon^{1 / 2} .
$$

Thus, we have the estimate

$$
\begin{aligned}
\inf _{w_{h} \in V_{e}^{h}}\left\|\mathfrak{u}^{\varepsilon}-w_{h}\right\|_{H^{1}\left(\Omega_{R}\right)} & \leqslant \kappa \varepsilon^{1 / 2}+\inf _{w_{h} \in V_{e}^{h}}\left\|\widetilde{u}^{\varepsilon}-w_{h}\right\|_{H^{1}\left(\Omega_{R}\right)}, \\
& \leqslant \kappa \varepsilon^{1 / 2}+\inf _{w_{h} \in V^{h}}\left\|\widetilde{u}^{\varepsilon}-\frac{-u_{0}(0)}{\ln \varepsilon-\lambda_{0}} \psi^{\varepsilon}-w_{h}\right\|_{H^{1}\left(\Omega_{R}\right)} .
\end{aligned}
$$

In the last term of this inequality, we put the additional shape function in order to "kill" the singularity of $\widetilde{u}^{\varepsilon}$. However, $\psi^{\varepsilon}$ does not reproduce exactly this singularity, so we introduce another 
function $\widetilde{\psi}^{\varepsilon}$ close to $\psi^{\varepsilon}$. We define it by

$$
\begin{aligned}
\widetilde{\psi}^{\varepsilon}(\mathbf{x}) & =\chi_{0}(\mathbf{x})\left(1-\chi_{1 / 2}^{\varepsilon}(\mathbf{x})\right) \frac{2}{\pi}\left(J_{0}(k|\mathbf{x}|)-1\right) \ln |\mathbf{x}| \\
& +\chi_{0}(\mathbf{x}) \mathbf{1}_{\Omega_{R} \backslash \overline{\Omega_{\varepsilon}^{i}}}(\mathbf{x})\left(\frac{2}{\pi} J_{0,0} \ln \frac{|\mathbf{x}|}{\varepsilon}+Y_{0,0}+l_{0}^{1}\left(V_{0}\right)\right) \\
& +\chi_{1 / 2}^{\varepsilon}(\mathbf{x}) \mathbf{1}_{\Omega_{R} \backslash \overline{\Omega_{\varepsilon}^{i}}}(\mathbf{x})\left(4 V_{0}^{\varepsilon}(\mathbf{x})-\left(\frac{2}{\pi} J_{0,0} \ln \frac{|\mathbf{x}|}{\varepsilon}+Y_{0,0}+l_{0}^{1}\left(V_{0}\right)\right)\right) .
\end{aligned}
$$

The error can be decomposed into two terms as follows

$$
\begin{aligned}
\inf _{w_{h} \in V_{e}^{h}}\left\|\widetilde{u}^{\varepsilon}-\frac{-u_{0}(0)}{\ln \varepsilon-\lambda_{0}} \psi^{\varepsilon}-w_{h}\right\|_{H^{1}\left(\Omega_{R}\right)} & \leqslant \inf _{w_{h} \in V^{h}}\left\|\widetilde{u}^{\varepsilon}-\frac{-\frac{\pi}{2} u_{0}(0)}{\ln \varepsilon-\lambda_{0}} \widetilde{\psi}^{\varepsilon}-w_{h}\right\|_{H^{1}\left(\Omega_{R}\right)} \\
& +\frac{\kappa}{\ln 1 / \varepsilon} \inf _{w_{h} \in V^{h}}\left\|\frac{2}{\pi} \psi^{\varepsilon}-\widetilde{\psi}^{\varepsilon}-w_{h}\right\|_{H^{1}\left(\Omega_{R}\right)} .
\end{aligned}
$$

The first term of the right hand side of this inequality can be upper bounded using Lemma A.3 (with a suitable constant),

$$
\inf _{w_{h} \in V^{h}}\left\|\widetilde{u}^{\varepsilon}-\frac{-\frac{\pi}{2} u_{0}(0)}{\ln \varepsilon-\lambda_{0}} \widetilde{\psi}^{\varepsilon}-w_{h}\right\|_{H^{1}\left(\Omega_{R}\right)} \leqslant \kappa\left(\varepsilon^{1 / 2}+h\right) .
$$

the second term itself can be split into two parts,

$$
\begin{aligned}
\frac{2}{\pi} \psi^{\varepsilon}-\widetilde{\psi}^{\varepsilon}= & -\chi_{0}(\mathbf{x})\left(1-\chi_{1 / 2}^{\varepsilon}(\mathbf{x})\right) \frac{2}{\pi}\left(J_{0}(k|\mathbf{x}|)-1\right) \ln |\mathbf{x}| \\
& +\chi_{0}(\mathbf{x})\left(1-\chi_{1 / 2}^{\varepsilon}(\mathbf{x})\right)\left(4 V_{0}^{\varepsilon}(\mathbf{x})-\left(\frac{2}{\pi} J_{0,0} \ln \frac{|\mathbf{x}|}{\varepsilon}+Y_{0,0}+l_{0}^{1}\left(V_{0}\right)\right)\right) .
\end{aligned}
$$

The first part can be bounded using classical consistancy estimates and

$$
\left\|\left(1-\chi_{1 / 2}^{\varepsilon}(\mathbf{x})\right)\left(J_{0}(k|\mathbf{x}|)-1\right) \ln |\mathbf{x}|\right\|_{H^{2}\left(\Omega_{R}\right)} \leqslant \kappa \ln 1 / \varepsilon,
$$

while the second part can be upper bounded using the technical Lemma A.2 in the appendix. We find

$$
\frac{1}{\ln 1 / \varepsilon} \inf _{w_{h} \in V_{e}^{h}}\left\|\frac{2}{\pi} \psi^{\varepsilon}-\widetilde{\psi}^{\varepsilon}-w_{h}\right\|_{H^{1}\left(\Omega_{R}\right)} \leqslant \kappa\left(\varepsilon^{1 / 2}+h\right) .
$$

It is now sufficient to gather all these previous estimates to obtain the existence of $\kappa>0$ independent of $\varepsilon$ such that

$$
\begin{aligned}
\inf _{w_{h} \in V_{e}^{h}}\left\|\mathfrak{u}^{\varepsilon}-w_{h}\right\|_{H^{1}\left(\Omega_{R}\right)} & \leqslant \kappa \varepsilon^{1 / 2}+\inf _{w_{h} \in V_{e}^{h}}\left\|\widetilde{u}^{\varepsilon}-\frac{-u_{0}(0)}{\ln \varepsilon-\lambda_{0}} \psi^{\varepsilon}-w_{h}\right\|_{H^{1}\left(\Omega_{R}\right)} \\
& \leqslant \kappa\left(\varepsilon^{1 / 2}+h\right) .
\end{aligned}
$$

Using Theorems 2.1 2.3. Inequality (4.2) and Theorem 4.1] we finally get the global estimate result for the difference between the solution of the initial problem (2.1) and the solution of the discrete problem [4.1.

\section{Corollary 4.1}

There exists a constant $\kappa>0$ independent of $h$ and $\varepsilon$ such that

$$
\left\|u^{\varepsilon}-u^{\varepsilon, h}\right\|_{H^{1}\left(\Omega_{R}\right)} \leqslant \kappa \sqrt{\ln 1 / \varepsilon}\left(\varepsilon^{1 / 2}+h\right) .
$$


Similarly, using Proposition 2.7 of [1] and Lemma 3.1] above, we can formulate a consistency result for Lagrange multipliers.

Corollary 4.2

There exists a constant $\kappa>0$ independent of $h$ and $\varepsilon$ such that

$$
\left|p^{\varepsilon}-p^{\varepsilon, h}\right| \leqslant \kappa \sqrt{\ln 1 / \varepsilon}\left(\varepsilon^{1 / 2}+h\right) .
$$

In particular, we obtain from Corollary 4.2 an estimate for the value of $p^{\varepsilon, h}$. Indeed, suppose that the couple $(h, \varepsilon)$ goes to 0 so that $\sqrt{\ln 1 / \varepsilon}\left(\varepsilon^{1 / 2}+h\right)$ also goes to 0 . For example this is verified when $\varepsilon=h^{1+\nu}$ for a given $\nu>0$ and corresponds to practical situations. Then this corollary joined with Equality (2.4) shows that $p^{\varepsilon, h} \sim p^{\varepsilon} \sim \kappa / \ln 1 / \varepsilon$.

The two last results suggest that the convergence is quite slow with $h$, since the upper bound appearing in these results is proportional to $h \sqrt{\ln 1 / \varepsilon}$, regardless of the order of the finite elements, with in addition a lack of consistency of $\varepsilon^{1 / 2}$. Concerning the lack of consistency, it seems that it is not possible to hope for a better result. But maybe the bound $h \sqrt{\ln 1 / \varepsilon}$ is not optimal. This is the subject of the next paragraph.

\subsection{Error estimate away from the obstacle}

The result of Corollary 4.1 is interesting because it yields consistency for the finite element method augmented with an additional shape function. In other words, the method is locking-free. However error estimate does not match the order $q$ of the finite element method since the bound is proportional to $h \sqrt{\ln 1 / \varepsilon}$. The next theorem shows that the error is really smaller in any region that excludes a fixed neighborhood of the obstacle.

\section{Theorem 4.2}

Consider an open set $\Omega_{0} \subset \Omega_{R}$ such that $\Omega_{0} \cap$ supp $\psi^{\varepsilon}=\emptyset$. then there exists $\kappa$ depending on $\Omega_{0}$ but independent of $\varepsilon$ and $h$ such that

$$
\left\|u^{\varepsilon}-u^{\varepsilon, h}\right\|_{H^{1}\left(\Omega_{0}\right)} \leqslant \kappa\left(\varepsilon+h^{q}+\left\|\mathfrak{u}^{\varepsilon}-u^{\varepsilon, h}\right\|_{H^{-p}\left(\Omega_{1}\right)}\right) .
$$

\section{Proof:}

Take two open sets $\Omega_{1}, \Omega_{1}^{\prime}$ such that $\Omega_{0} \subset \subset \Omega_{1} \subset \subset \Omega_{1}^{\prime} \subset \Omega_{R}$ and $\Omega_{1}^{\prime} \cap \operatorname{supp} \psi^{\varepsilon}=\emptyset$. First, by triangular inequality an Theorems 2.2 and 2.4 we can find $\kappa$ independent of $\varepsilon$ and $h$ such that

$$
\left\|u^{\varepsilon}-u^{\varepsilon, h}\right\|_{H^{1}\left(\Omega_{0}\right)} \leqslant\left\|u^{\varepsilon}-\mathfrak{u}^{\varepsilon}\right\|_{H^{1}\left(\Omega_{0}\right)}+\left\|\mathfrak{u}^{\varepsilon}-u^{\varepsilon, h}\right\|_{H^{1}\left(\Omega_{0}\right)} \leqslant \kappa \varepsilon+\left\|\mathfrak{u}^{\varepsilon}-u^{\varepsilon, h}\right\|_{H^{1}\left(\Omega_{0}\right)} .
$$

Moreover, a theorem from Nitsche and Schatz A.1 that we recall in appendix, yields

$$
\left\|\mathfrak{u}^{\varepsilon}-u^{\varepsilon, h}\right\|_{H^{1}\left(\Omega_{0}\right)} \leqslant \kappa\left(\varepsilon+h^{q}\left\|\mathfrak{u}^{\varepsilon}\right\|_{H^{q+1}\left(\Omega_{1}\right)}+\left\|\mathfrak{u}^{\varepsilon}-u^{\varepsilon, h}\right\|_{H^{-p}\left(\Omega_{1}\right)}\right) .
$$

The only remaining problem consists in bounding $\left\|\mathfrak{u}^{\varepsilon}\right\|_{H^{q+1}\left(\Omega_{1}\right)}$. This can be done using a cut-off function in the equations defining $\mathfrak{u}^{\varepsilon}$ and using elliptic regularity results.

\subsection{Numerical experiments on the augmented formulation}

We now come back to the numerical experiment presented in paragraph 3.2 We carried out the same experiment replacing the standard finite element space by the augmented one, thus solving (4.1). Except this difference, the situation is the same: $\Omega_{R}=D(0,3)$, modified absorbing boundary 
condition, same incident field, quadrangular finite elements of order 3 , wavelength $\lambda=1$, etc... In this case the additional shape function takes the form

$$
\psi^{\varepsilon}(\mathbf{x})=\chi_{0}(\mathbf{x}) \ln \left(\frac{|\mathbf{x}|}{\varepsilon}\right) \mathbf{1}_{|\mathbf{x}|>\varepsilon} \quad \text { with } \quad \chi_{0}(\mathbf{x})= \begin{cases}1 & \text { if }|\mathbf{x}| \leqslant r_{0} / 3 \\ P(|\mathbf{x}|) & \text { if } \quad r_{0} / 3 \leqslant|\mathbf{x}| \leqslant 2 r_{0} / 3 \\ 0 & \text { if } \quad|\mathbf{x}| \geqslant 2 r_{0} / 3 .\end{cases}
$$

Number $r_{0}$ is a parameter of the method; in practice, we took $r_{0}=1 . P$ is a polynomial providing regularity of the shape function between the regions $|\mathbf{x}| \leqslant r_{0} / 3$ and $|\mathbf{x}| \geqslant 2 r_{0} / 3$. The order of regularity $C^{0}, C^{1}$, etc ... is also a parameter of the method. For example with a $C^{1}$ cut-off function, $P$ will be the unique polynomial of order 3 satisfying $P\left(r_{0} / 3\right)=1, P^{\prime}\left(r_{0} / 3\right)=0, P\left(2 r_{0} / 3\right)=0$ and $P^{\prime}\left(2 r_{0} / 3\right)=0$. The additional shape function and the cut-off function are radial. In order well to visualize their shape, we have represented their transversal section in Figure 5 for $\varepsilon=0.06$ and $r_{0}=0.6$.

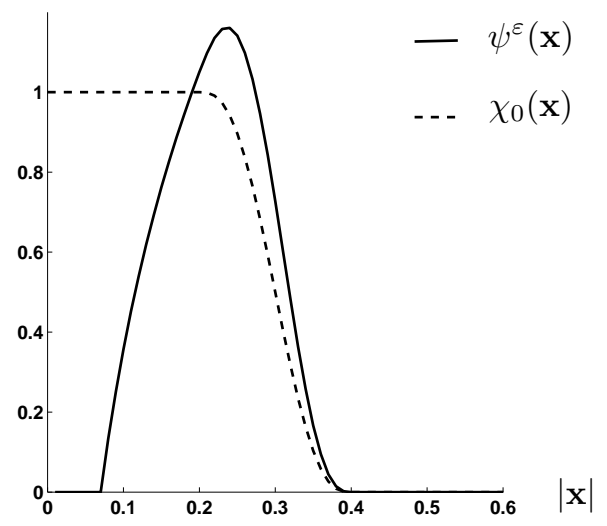

Figure 5: Graph of the additional function $\psi^{\varepsilon}$

In Figure 6] we have represented the relative errors obtained with this experiment. 


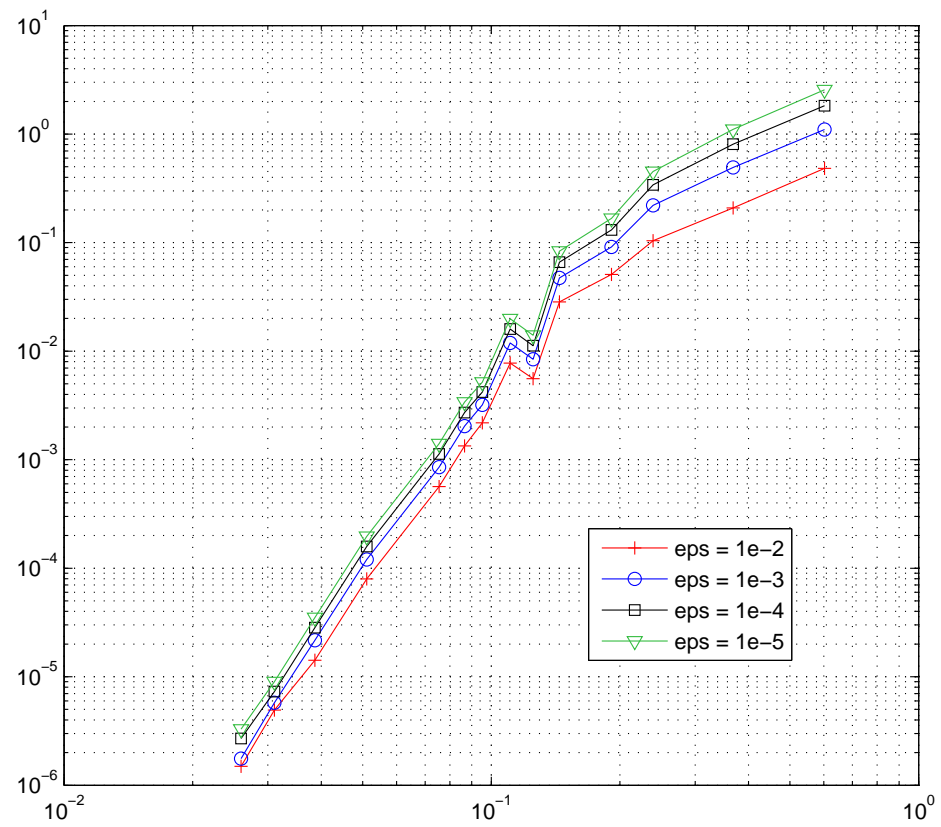

Figure 6: Relative error versus step of the mesh

The results are far better than in Section 3.2 for at least three reasons. First, the precision is much sharper since we obtain a relative error of $10^{-5}$ for about 100 points per wavelengths, and this holds regardless of the value of $\varepsilon$ we choose. Moreover the graph goes up very slowly as $\varepsilon$ goes to 0 . Finally, the rates of convergence are in accordance with the order 3 of the finite element method.

\begin{tabular}{|c|c|}
\hline$\varepsilon / \lambda$ & slope \\
\hline $10^{-2}$ & 3.8883 \\
\hline $10^{-3}$ & 4.1136 \\
\hline $10^{-4}$ & 4.2392 \\
\hline $10^{-5}$ & 4.3103 \\
\hline
\end{tabular}

We have also represented the relative errors for the Lagrange multipliers. They are represented in Figure 7 


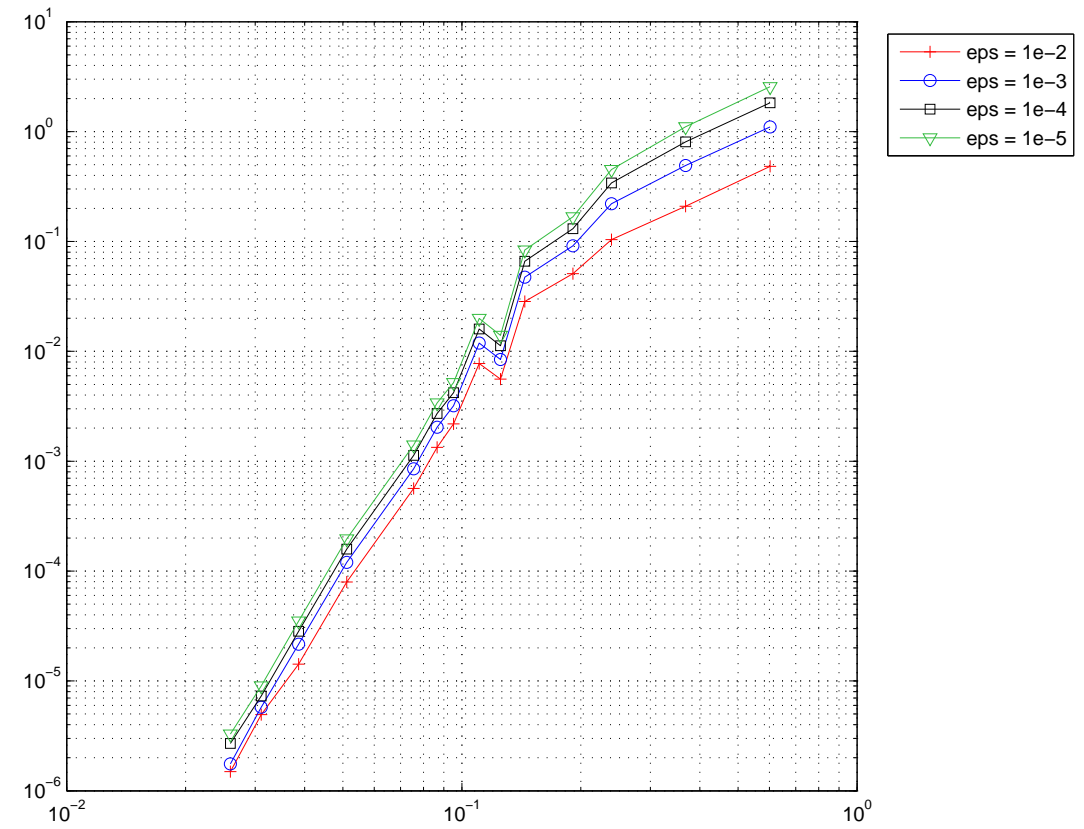

Figure 7: Relative error on Lagrange multipliers versus step of the mesh

The rates of convergence for this figure are given in the table below.

\begin{tabular}{|c|c|}
\hline$\varepsilon / \lambda$ & slope \\
\hline $10^{-2}$ & 4.2939 \\
\hline $10^{-3}$ & 4.5092 \\
\hline $10^{-4}$ & 4.5752 \\
\hline $10^{-5}$ & 4.6161 \\
\hline
\end{tabular}

\section{Study of the additional shape function}

In order well to understand the effect of the additional shape function $\psi^{\varepsilon}$ of the augmented scheme (4.1), we will study it in detail. For the rest of this document we will suppose that the obstacle is a small disk of radius $\varepsilon$, so that the additional shape function admits a very simple expression,

$$
\psi^{\varepsilon}(\mathbf{x})=\chi_{0}(\mathbf{x}) \ln \left(\frac{|\mathbf{x}|}{\varepsilon}\right) \mathbf{1}_{|\mathbf{x}|>\varepsilon} .
$$

The function $\psi^{\varepsilon}$ is only partially approximated by the functions of the standard finite element approximation space, and this is the reason why the scheme (4.1) is more precise than (3.1). Let us introduce two additional notations. Given $v \in H^{1}\left(\Omega_{R}\right)$, we will denote $\mathcal{P}_{h}(v)$ the unique element of the space $V^{h}$ satisfying

$$
a\left(\mathcal{P}_{h}(v), w_{h}\right)=a\left(v, w_{h}\right), \quad \forall w_{h} \in V^{h}
$$

Moreover we define

$$
\delta_{h}(v)=v-\mathcal{P}_{h}(v) .
$$

Remind that, according to a classical result of consistency for finite elements, if $v \in H^{1+\nu}\left(\Omega_{R}\right)$ with $\nu>0$ then $\mathcal{P}_{h}(v)$ is a good approximation of $v$ in the sense that there exists $\kappa>0$ independent 
of $h$ such that $\left\|\delta_{h}(v)\right\|_{H^{1}\left(\Omega_{R}\right)} \leqslant \kappa\|v\|_{H^{1+\nu}\left(\Omega_{R}\right)} h^{\nu}$. We can show moreover that $\psi^{\varepsilon} \in H^{1+\nu}\left(\Omega_{R}\right)$ for any $\nu<\frac{1}{2}$. We can expect that the more $h$ is close to 0 , the better $\psi^{\varepsilon}$ is approximated by elements of $V^{h}$. We see that in order to gain understanding of both the situation and the effect of the additional shape function, it is necessary to know the behavior of $\left\|\delta_{h}\left(\psi^{\varepsilon}\right)\right\|_{H^{1}\left(\Omega_{R}\right)}$. The next section is dedicated to this question. We proceed in two steps. First, we will provide an estimate for $\left\|\delta_{h}\left(\psi^{\varepsilon}\right)\right\|_{H^{1}\left(\Omega_{R}\right)}$, which is a global estimate. Secondly, we provide an estimate in $H^{1}$ only on a region $\mathcal{O}$ that excludes a fixed neighborhood of the small obstacle: this is a local estimate. Such estimates will also be provided for the $L^{2}$ norm

\subsection{Global estimate}

We will first see that $\psi^{\varepsilon}$ is not well approximated in $H^{1}$ norm on the whole domain. We start by formulating a technical lemma. In order to make the presentation more clear, the proof of this result is delayed to the next section. Here is what we obtain when we apply classical technique for the consistency of finite elements.

\section{Lemma 5.1}

There exists a constant $\kappa>0$ independent of $h$ and $\varepsilon$, such that $\left\|\delta_{h}\left(\psi^{\varepsilon}\right)\right\|_{H^{1}\left(\Omega_{R}\right)} \leqslant \kappa\left(1+h^{q} \ln \frac{h}{\varepsilon}\right)$.

This lemma suggests us that the approximation of the additional shape function by finite elements is not very good. Indeed the next lemma will show that it is useless to hope that $\left\|\delta_{h}\left(\psi^{\varepsilon}\right)\right\|_{H^{1}\left(\Omega_{R}\right)}$ goes to 0 .

\section{Lemma 5.2}

Under assumptions $\boldsymbol{H} \mathbf{1}$ and $\boldsymbol{H} \boldsymbol{2}$, there exists $\kappa>0$ independent of $h$ and $\varepsilon$ such that $\left\|\delta_{h}\left(\psi^{\varepsilon}\right)\right\|_{H^{1}\left(\Omega_{R}\right)}>\kappa$.

\section{Proof:}

Take two fixed constants $\kappa_{-}, \kappa_{+}>0$ such that $\kappa<\kappa_{-}<\kappa_{-}+3<\kappa_{+}$. We know that for any $h$ small enough, there exists an element $K$ of the mesh contained in the annulus $\left\{\mathbf{x} \in \Omega_{R}\right.$ t.q $\kappa_{-} h<$ $\left.|\mathbf{x}|<\kappa_{+} h\right\}$. We denote this element $K(h)$. In order to prove this lemma, it is sufficient to prove that

$$
\exists \kappa_{2}>0 \text { s.t } \forall h, \quad \int_{K(h)}\left|\nabla \delta_{h}\left(\psi^{\varepsilon}\right)\right|^{2} d \mathbf{x}>\kappa_{2} .
$$

For any $h$, we denote by $F^{h}: \widehat{K} \rightarrow K$ the transformation that takes the reference element to $K(h)$. Then $F^{h}=\left(F_{1}^{h}, F_{2}^{h}\right)$ with $F_{i}^{h}(\widehat{\mathbf{x}}) \in \mathbb{P}_{1}\left(\right.$ where $\left.\mathbb{Q}_{1}\right), i=1,2$. Under the assumptions formulated previously, there always holds $\forall \widehat{\mathbf{x}} \in \widehat{K} \forall h>0, \kappa_{-}<\left|\frac{1}{h} F^{h}(\widehat{\mathbf{x}})\right|<\kappa_{+}$. Then it is sufficient to prove that

$$
\exists \kappa_{2}>0 \text { s.t } \forall h, \inf _{P \in P_{q}} \int_{\widehat{K}}\left|\frac{F_{1}^{h}(\widehat{\mathbf{x}})}{\left|F^{h}(\widehat{\mathbf{x}})\right|^{2}}-P(\widehat{\mathbf{x}})\right|^{2}\left|\operatorname{Jac}\left(F^{h}\right)\right| d \widehat{\mathbf{x}}>\kappa_{2} .
$$

Proceed by contradiction and suppose there exists a sequence $h_{n} \rightarrow 0$ and a sequence of polynomials of bounded degree $P_{n} \in \mathbb{P}_{q}$ such that

$$
\int_{\widehat{K}}\left|\frac{F_{1}^{h_{n}}(\widehat{\mathbf{x}})}{\left|F^{h_{n}}(\widehat{\mathbf{x}})\right|^{2}}-\frac{1}{h_{n}} P_{n}(\widehat{\mathbf{x}})\right|^{2}\left|\operatorname{Jac}\left(F^{h_{n}}\right)\right| d \widehat{\mathbf{x}} \rightarrow 0 \quad \text { when } \quad n \rightarrow+\infty .
$$

Then we know (c.f. for instance [7]) that there exists a constant $\kappa_{3}>0$ such that for any $h$, $\left|\operatorname{Jac}\left(F^{h}\right)\right|>\kappa_{3} h^{2}$ which implies

$$
\int_{\widehat{K}}\left|\frac{F_{1}^{h_{n}}(\widehat{\mathbf{x}})}{\left|F^{h_{n}}(\widehat{\mathbf{x}})\right|^{2}}-\frac{1}{h_{n}} P_{n}(\widehat{\mathbf{x}})\right|^{2}\left|\operatorname{Jac}\left(F^{h_{n}}\right)\right| d \widehat{\mathbf{x}}>\int_{\widehat{K}}\left|\frac{\frac{1}{h_{n}} F_{1}^{h_{n}}(\widehat{\mathbf{x}})}{\left|\frac{1}{h_{n}} F^{h_{n}}(\widehat{\mathbf{x}})\right|^{2}}-P_{n}(\widehat{\mathbf{x}})\right|^{2} d \widehat{\mathbf{x}}
$$

Since $\forall n,\left\|\frac{1}{h_{n}} F^{h_{n}}\right\|_{\widehat{K}}<\kappa_{+}$, we conclude that the sequence $\left(\frac{1}{h_{n}} F^{h_{n}}\right)_{n}$ is bounded in $P_{1}^{2}$ (or in $\left.Q_{1}^{2}\right)$. As this space is of finite dimension, extracting a subsequence if necessary, we can assume that 
$\left(\frac{1}{h_{n}} F^{h_{n}}\right)_{n}$ converges, say toward $F=\left(F_{1}, F_{2}\right) \in P_{1}^{2}$ (or $\left.\in Q_{1}^{2}\right)$. Then, $\forall \widehat{\mathbf{x}} \in \widehat{K}, \kappa_{-}<|F(\widehat{\mathbf{x}})|<$ $\kappa_{+}$, which implies that $\frac{F_{1}}{|F|^{2}}$ is well defined, different from zero and $C^{\infty}$ on $\widehat{K}$. Moreover $h_{n} \frac{F_{1}^{h_{n}}}{\left|F^{h_{n}}\right|^{2}}$ uniformly converges toward $\frac{F_{1}}{|F|^{2}}$ on $\widehat{K}$. Similarly we can show that $P_{n}$ uniformly converges toward a polynomial with the same degree. Going to the limit, we finally obtain

$$
\int_{\widehat{K}}\left|\frac{F_{1}(\widehat{\mathbf{x}})}{|F(\widehat{\mathbf{x}})|^{2}}-P(\widehat{\mathbf{x}})\right|^{2} d \widehat{\mathbf{x}}=0 \quad \Rightarrow \quad \frac{F_{1}(\widehat{\mathbf{x}})}{|F(\widehat{\mathbf{x}})|^{2}}=P(\widehat{\mathbf{x}})
$$

Suppose first that $P$ is not constant. This leads to a contradiction since

$$
\frac{\left|F_{1}(\widehat{\mathbf{x}})\right|}{|F(\widehat{\mathbf{x}})|^{2}} \underset{|\widehat{\mathbf{x}}| \rightarrow+\infty}{\rightarrow} 0 \quad \text { whereas } \quad|P(\widehat{\mathbf{x}})| \underset{|\widehat{\mathbf{x}}| \rightarrow+\infty}{\rightarrow}+\infty .
$$

If we suppose that $P$ is constant, this implies that $F$ is also constant, and this yields another contradiction since by continuity $|\operatorname{Jac}(F)|>\kappa_{3}>0$. To conclude, contradictions gives us the result.

We see that there is no global convergence in $H^{1}$ norm. However in $L^{2}$ there is a slow convergence as we can see with the following lemma.

Lemma 5.3 There exists a constant $\kappa>0$ independent of $h$ and $\varepsilon$, such that $\left\|\delta_{h}\left(\psi^{\varepsilon}\right)\right\|_{L^{2}\left(\Omega_{R}\right)} \leqslant$ $\kappa h\left(1+h^{q} \ln \frac{h}{\varepsilon}\right)$.

\section{Proof:}

We obtain this result by a classical duality method. Given $v \in L^{2}\left(\Omega_{R}\right)$ we define $w_{v}$ as the unique element of $H^{1}\left(\Omega_{R}\right)$ satisfying $a\left(w_{v}, w\right)=\int_{\Omega_{R}} v w, \forall w \in H^{1}\left(\Omega_{R}\right)$. Then the following usual calculus holds

$$
\begin{aligned}
\left\|\delta_{h}\left(\psi^{\varepsilon}\right)\right\|_{L^{2}\left(\Omega_{R}\right)} & =\sup _{v \in L^{2}\left(\Omega_{R}\right)} \frac{\int_{\Omega_{R}} \delta_{h}\left(\psi^{\varepsilon}\right) v}{\|v\|_{L^{2}\left(\Omega_{R}\right)}}=\sup _{v \in L^{2}\left(\Omega_{R}\right)} \frac{a\left(\delta_{h}\left(\psi^{\varepsilon}\right), w_{v}\right)}{\|v\|_{L^{2}\left(\Omega_{R}\right)}} \\
& =\sup _{v \in L^{2}\left(\Omega_{R}\right)} \frac{a\left(\delta_{h}\left(\psi^{\varepsilon}\right), w_{v}-\mathcal{P}_{h}\left(w_{v}\right)\right)}{\|v\|_{L^{2}\left(\Omega_{R}\right)}} \\
& \leqslant \kappa h\left\|\delta_{h}\left(\psi^{\varepsilon}\right)\right\|_{H^{1}\left(\Omega_{R}\right)},
\end{aligned}
$$

whence the result by Lemma 5.1

\subsection{Local estimate}

We are now interested in the convergence of $\mathcal{P}_{h}\left(\psi^{\varepsilon}\right)$ toward $\psi^{\varepsilon}$ in a region that excludes a fixed neighborhood of the obstacle. As $\psi^{\varepsilon}$ is very smooth in such a region, there should have faster convergence. Indeed take two open sets $\Omega_{0} \subset \subset \Omega_{1} \subset \subset \Omega_{R} \backslash \overline{\Omega_{\varepsilon}^{i}}$. 


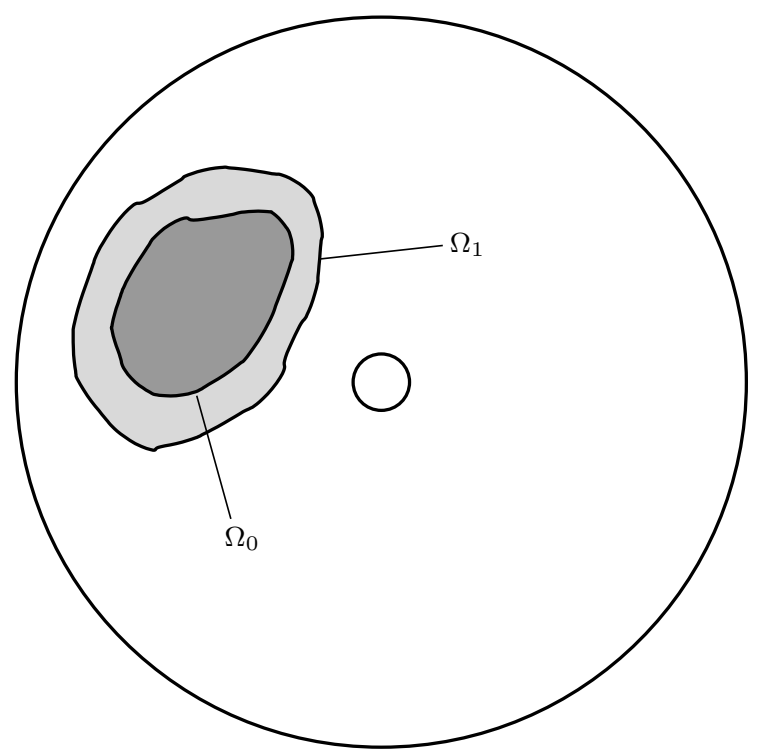

Figure 8: Sub-domains for the local estimate

We wish to bound $\left\|\psi^{\varepsilon}-\mathcal{P}_{h}\left(\psi^{\varepsilon}\right)\right\|_{H^{1}\left(\Omega_{0}\right)}$ and $\left\|\psi^{\varepsilon}-\mathcal{P}_{h}\left(\psi^{\varepsilon}\right)\right\|_{L^{2}\left(\Omega_{0}\right)}$ depending on the behavior of $\psi^{\varepsilon}$ in $\Omega_{1}$. For this analysis, we will use a theorem of Nitsche and Schatz [11]. We recall this result in appendix.

\section{Lemma 5.4}

There exists $\kappa>0$ independent of $\varepsilon$ and $h$ such that $\left\|\delta_{h}\left(\psi^{\varepsilon}\right)\right\|_{H^{1}\left(\Omega_{0}\right)} \leqslant \kappa h^{q}\left(1+\ln \frac{h}{\varepsilon}\right)$.

Proof:

According to Nitsche and Schatz' theorem, there exists $\kappa>0$ independent of $\varepsilon$ and $h$ such that

$$
\left\|\delta_{h}\left(\psi^{\varepsilon}\right)\right\|_{H^{1}\left(\Omega_{0}\right)} \leqslant \kappa\left(h^{q}\left\|\psi^{\varepsilon}\right\|_{H^{q+1}\left(\Omega_{1}\right)}+\left\|\delta_{h}\left(\psi^{\varepsilon}\right)\right\|_{H^{-q+1}\left(\Omega_{1}\right)}\right) .
$$

Take $v \in H^{q-1}\left(\Omega_{R}\right)$ and consider the unique functions $w_{v} \in H^{1}\left(\Omega_{R}\right)$ and $w_{v}^{h} \in V^{h}$ satisfying

$$
a\left(w_{v}, w\right)=\int_{\Omega_{R}} w v \quad \forall w \in H^{1}\left(\Omega_{R}\right) \quad \text { et } \quad a\left(w_{v}^{h}, w_{h}\right)=\int_{\Omega_{R}} w_{h} v \quad \forall w_{h} \in V^{h} .
$$

Then

$$
\begin{aligned}
\int_{\Omega_{R}} \delta_{h}\left(\psi^{\varepsilon}\right) v & =a\left(\delta_{h}\left(\psi^{\varepsilon}\right), w_{v}\right)=a\left(\delta_{h}\left(\psi^{\varepsilon}\right), w_{v}-w_{v}^{h}\right) \\
& \leqslant \kappa\left\|\delta_{h}\left(\psi^{\varepsilon}\right)\right\|_{H^{1}\left(\Omega_{R}\right)}\left\|w_{v}-w_{v}^{h}\right\|_{H^{1}\left(\Omega_{R}\right)} \\
& \leqslant \kappa\left\|\delta_{h}\left(\psi^{\varepsilon}\right)\right\|_{H^{1}\left(\Omega_{R}\right)}\left\|w_{v}\right\|_{H^{q+1}\left(\Omega_{R}\right)} h^{q} \leqslant \kappa\left\|\delta_{h}\left(\psi^{\varepsilon}\right)\right\|_{H^{1}\left(\Omega_{R}\right)}\|v\|_{H^{q-1}\left(\Omega_{R}\right)} h^{q},
\end{aligned}
$$

which implies

$$
\begin{aligned}
\left\|\delta_{h}\left(\psi^{\varepsilon}\right)\right\|_{H^{-q+1}\left(\Omega_{1}\right)} & =\sup _{v \in H_{0}^{q-1}\left(\Omega_{1}\right)} \frac{\int_{\Omega_{R}} \delta_{h}\left(\psi^{\varepsilon}\right) v}{\|v\|_{H^{q-1}\left(\Omega_{R}\right)}} \\
& \leqslant \sup _{v \in H^{q-1}\left(\Omega_{R}\right)} \frac{\int_{\Omega_{R}} \delta_{h}\left(\psi^{\varepsilon}\right) v}{\|v\|_{H^{q-1}\left(\Omega_{R}\right)}} \leqslant \kappa\left\|\delta_{h}\left(\psi^{\varepsilon}\right)\right\|_{H^{1}\left(\Omega_{R}\right)} h^{q} .
\end{aligned}
$$

Finally, we know that

$$
\left\|\psi^{\varepsilon}\right\|_{H^{q+1}\left(\Omega_{1}\right)} \leqslant \kappa\left(1+\ln \frac{h}{\varepsilon}\right) \quad \text { and } \quad\left\|\delta_{h}\left(\psi^{\varepsilon}\right)\right\|_{H^{1}\left(\Omega_{R}\right)} \leqslant \kappa\left(1+\ln \frac{h}{\varepsilon}\right)
$$


which gives us the final result.

We can apply the same techniques using the second part of Nitsche and Schatz' theorem in order to obtain local estimate in $L^{2}$ norm.

\section{Corollary 5.1}

There exists $\kappa>0$ independent of $\varepsilon$ and $h$ such that $\left\|\delta_{h}\left(\psi^{\varepsilon}\right)\right\|_{L^{2}\left(\Omega_{0}\right)} \leqslant \kappa h^{q+1}\left(1+\ln \frac{h}{\varepsilon}\right)$.

Lemma 5.4 and corollary [5.1 yield estimates on an interior domain $\Omega_{0}$ such that $\Omega_{0} \subset \subset \Omega_{R}$. This implies in particular that $\Omega_{0} \cap \Gamma_{R}=\emptyset$. So we still do not have any estimate in the neighborhood of the exterior boundary $\Gamma_{R}$. This is the subject of the next lemma. We will bound the error in $\Omega_{R} \backslash \overline{\Omega_{R^{\prime}}}$ for $2 \varepsilon<R^{\prime}<R$. We will suppose that $R-R^{\prime}$ is small enough for guarantying that $\psi^{\varepsilon}=0$ on $\Omega_{R} \backslash \Omega_{R^{\prime}}$.

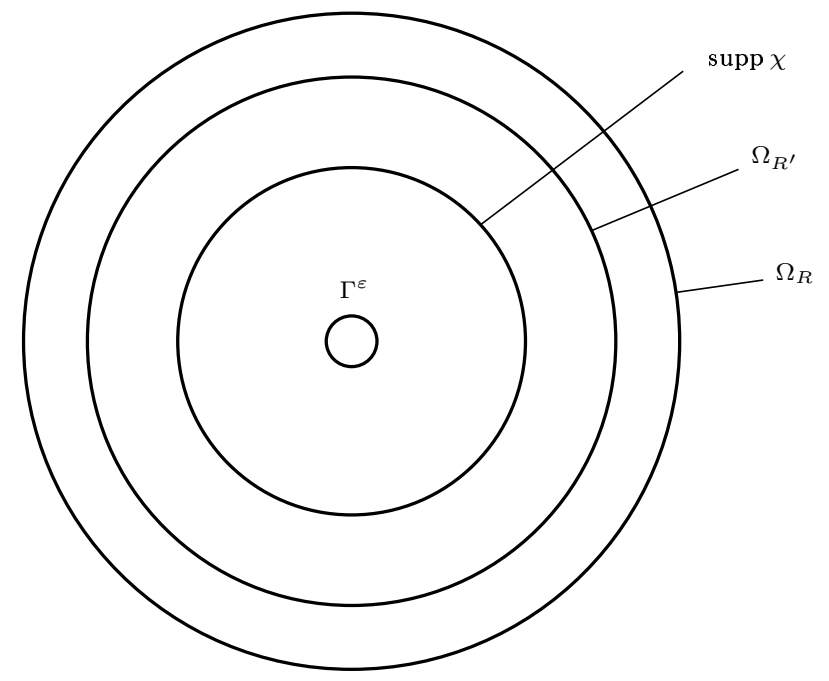

Figure 9: Sub-domain $\Omega_{R} \backslash \Omega_{R^{\prime}}$ for the local estimate close to the boundary.

\section{Lemma 5.5}

There exists $\kappa>0$ independent of $\varepsilon$ and $h$ such that

$$
\left\|\delta_{h}\left(\psi^{\varepsilon}\right)\right\|_{H^{1}\left(\Omega_{R} \backslash \Omega_{R^{\prime}}\right)}=\left\|\mathcal{P}_{h}\left(\psi^{\varepsilon}\right)\right\|_{H^{1}\left(\Omega_{R} \backslash \Omega_{R^{\prime}}\right)} \leqslant \kappa h^{q}\left(1+\ln \frac{h}{\varepsilon}\right) .
$$

\section{Proof:}

Take $\tilde{\chi} \in C^{\infty}\left(\mathbb{R}_{+}\right)$such that $\tilde{\chi}(r)=0$ if $0 \leqslant r \leqslant R^{\prime} / 2$, and $\tilde{\chi}(r)=1$ if $r \geqslant R^{\prime}$, and $\tilde{\chi}$ is a growing function. For $h$ small enough, there exists an open set $\mathcal{O} \subset \subset \Omega_{R} \backslash \overline{\Omega_{\varepsilon}^{i}}$ independent of $h$ such that $\operatorname{supp} \nabla \tilde{\chi} \subset \mathcal{O}$ and $\forall v_{h} \in V^{h}, \operatorname{supp}\left(\tilde{\chi} v_{h}-\Pi_{h}\left(\tilde{\chi} v_{h}\right)\right) \subset \mathcal{O}$, and such that $\mathcal{O} \cap \operatorname{supp} \chi=\emptyset$. Then by Cea's lemma, there exists $\kappa>0$ independent of $\varepsilon$ and $h$ such that

$$
\begin{aligned}
\left\|\widetilde{\chi} \mathcal{P}_{h}\left(\psi^{\varepsilon}\right)-\mathcal{P}_{h}\left(\tilde{\chi} \mathcal{P}_{h}\left(\psi^{\varepsilon}\right)\right)\right\|_{H^{1}\left(\Omega_{R}\right)} & \leqslant \kappa\left\|\widetilde{\chi} \mathcal{P}_{h}\left(\psi^{\varepsilon}\right)-\Pi_{h}\left(\tilde{\chi} \mathcal{P}_{h}\left(\psi^{\varepsilon}\right)\right)\right\|_{H^{1}\left(\Omega_{R}\right)} \\
& \leqslant \kappa\left\|\widetilde{\chi} \mathcal{P}_{h}\left(\psi^{\varepsilon}\right)-\Pi_{h}\left(\widetilde{\chi} \mathcal{P}_{h}\left(\psi^{\varepsilon}\right)\right)\right\|_{H^{1}(\mathcal{O})} \\
& \leqslant \kappa\left\|\widetilde{\chi} \mathcal{P}_{h}\left(\psi^{\varepsilon}\right)\right\|_{H^{1}(\mathcal{O})} \leqslant \kappa\left\|\mathcal{P}_{h}\left(\psi^{\varepsilon}\right)\right\|_{H^{1}(\mathcal{O})} \\
\left\|\widetilde{\chi} \mathcal{P}_{h}\left(\psi^{\varepsilon}\right)-\mathcal{P}_{h}\left(\widetilde{\chi} \mathcal{P}_{h}\left(\psi^{\varepsilon}\right)\right)\right\|_{H^{1}\left(\Omega_{R}\right)} & \leqslant \kappa h^{q}\left(1+\ln \frac{h}{\varepsilon}\right) .
\end{aligned}
$$


In the last inequality we used lemma 5.4 Moreover, by definition of $\mathcal{P}_{h}$, for any $v_{h} \in V^{h}$, we have

$$
\begin{aligned}
a\left(\mathcal{P}_{h}\left(\widetilde{\chi} \mathcal{P}_{h}\left(\psi^{\varepsilon}\right)\right), v_{h}\right) & =a\left(\tilde{\chi} \mathcal{P}_{h}\left(\psi^{\varepsilon}\right), v_{h}\right) \\
& =\int_{\Omega_{R}}\left(\mathcal{P}_{h}\left(\psi^{\varepsilon}\right) \nabla \tilde{\chi} \cdot \nabla v_{h}-v_{h} \nabla \widetilde{\chi} \cdot \nabla \mathcal{P}_{h}\left(\psi^{\varepsilon}\right)\right)+a\left(\mathcal{P}_{h}\left(\psi^{\varepsilon}\right), \widetilde{\chi} v_{h}\right) .
\end{aligned}
$$

For $h$ small enough, note that $\operatorname{supp}\left(\Pi_{h} \widetilde{\chi} v_{h}\right) \subset \Omega_{R} \backslash \overline{\operatorname{supp} \psi^{\varepsilon}}$. As a consequence, $a\left(\mathcal{P}_{h}\left(\psi^{\varepsilon}\right), \Pi_{h} \widetilde{\chi} v_{h}\right)$ $=a\left(\psi^{\varepsilon}, \Pi_{h} \widetilde{\chi} v_{h}\right)=0$ what implies

$$
a\left(\mathcal{P}_{h}\left(\psi^{\varepsilon}\right), \widetilde{\chi} v_{h}\right)=a\left(\mathcal{P}_{h}\left(\psi^{\varepsilon}\right), \widetilde{\chi} v_{h}-\Pi_{h} \widetilde{\chi} v_{h}\right) .
$$

As $\operatorname{supp} \nabla \widetilde{\chi} \subset \mathcal{O}$ and $\operatorname{supp}\left(\widetilde{\chi} v_{h}-\Pi_{h}\left(\widetilde{\chi} v_{h}\right)\right) \subset \mathcal{O}$, we can obtain $\kappa>0$ such that

$$
\begin{aligned}
& \left|\int_{\Omega_{R}}\left(\mathcal{P}_{h}\left(\psi^{\varepsilon}\right) \nabla \tilde{\chi} \cdot \nabla v_{h}-v_{h} \nabla \tilde{\chi} \cdot \nabla \mathcal{P}_{h}\left(\psi^{\varepsilon}\right)\right)\right| \leqslant \kappa\left\|\mathcal{P}_{h}\left(\psi^{\varepsilon}\right)\right\|_{H^{1}(\mathcal{O})}\left\|v_{h}\right\|_{H^{1}\left(\Omega_{R}\right)}, \\
& \left|a\left(\mathcal{P}_{h}\left(\psi^{\varepsilon}\right), \tilde{\chi} v_{h}-\Pi_{h} \tilde{\chi} v_{h}\right)\right| \leqslant \kappa\left\|\mathcal{P}_{h}\left(\psi^{\varepsilon}\right)\right\|_{H^{1}(\mathcal{O})}\left\|v_{h}\right\|_{H^{1}\left(\Omega_{R}\right)} .
\end{aligned}
$$

Using inf - sup condition on $a$, we obtain

$$
\left\|\mathcal{P}_{h}\left(\widetilde{\chi} \mathcal{P}_{h}\left(\psi^{\varepsilon}\right)\right)\right\|_{H^{1}\left(\Omega_{R}\right)} \leqslant \kappa\left\|\mathcal{P}_{h}\left(\psi^{\varepsilon}\right)\right\|_{H^{1}(\mathcal{O})} \leqslant \kappa h^{q}\left(1+\ln \frac{h}{\varepsilon}\right) .
$$

Finally note that

$$
\begin{aligned}
\left\|\mathcal{P}_{h}\left(\psi^{\varepsilon}\right)\right\|_{H^{1}\left(\Omega_{R} \backslash \Omega_{R^{\prime}}\right)} & \leqslant\left\|\widetilde{\chi} \mathcal{P}_{h}\left(\psi^{\varepsilon}\right)\right\|_{H^{1}\left(\Omega_{R}\right)} \\
& \leqslant\left\|\widetilde{\chi} \mathcal{P}_{h}\left(\psi^{\varepsilon}\right)-\mathcal{P}_{h} \tilde{\chi} \mathcal{P}_{h}\left(\psi^{\varepsilon}\right)\right\|_{H^{1}\left(\Omega_{R}\right)}+\left\|\mathcal{P}_{h} \tilde{\chi} \mathcal{P}_{h}\left(\psi^{\varepsilon}\right)\right\|_{H^{1}\left(\Omega_{R}\right)},
\end{aligned}
$$

and this along with the previous inequalities provides the conclusion of the proof.

The previous result lead us to the following conclusions. $\left(\varphi_{i}\right)_{i=1 \ldots I} \cup\left(\delta_{h}\left(\psi^{\varepsilon}\right)\right)$ form a basis of $V_{\mathbf{e}}^{h}$. Coming back to the solution $u^{\varepsilon, h} \in V_{\mathbf{e}}^{h}$ of problem (4.1), we can decompose it on this basis:

$$
u^{\varepsilon, h}=u_{\mathbf{r}}^{\varepsilon, h}+p_{\mathbf{s}}^{\varepsilon, h} \delta_{h}\left(\psi^{\varepsilon}\right),
$$

where $u_{\mathbf{r}}^{\varepsilon, h} \in V^{h}$. Suppose $p_{\mathbf{s}}^{\varepsilon, h}$ is bounded (with respect to $\varepsilon$ and $h$ ), then Lemma [5.5 states that $u^{\varepsilon, h} \simeq u_{\mathbf{r}}^{\varepsilon, h}$ away from the small obstacle $\Omega_{\varepsilon}^{i}$.

\subsection{Illustration}

We numerically observed the effect of the operator $\delta_{h}$ on $\psi^{\varepsilon}$. The result is represented in the figure below.

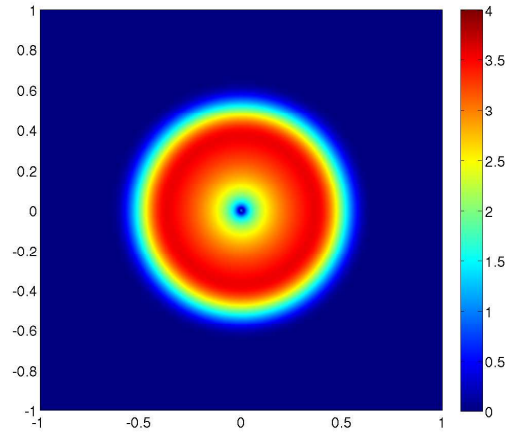

$\Re e\left\{\psi^{\varepsilon}\right\}$

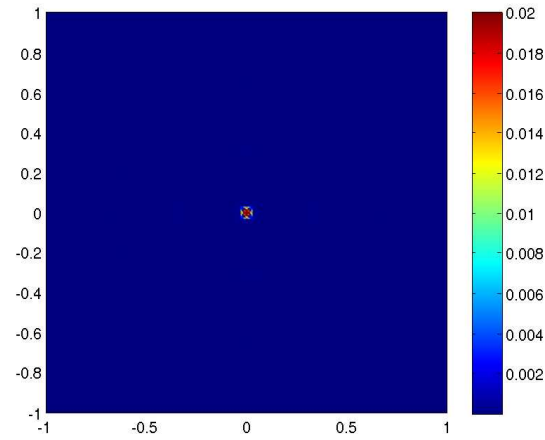

$\Re e\left\{\delta_{h}\left(\psi^{\varepsilon}\right)\right\}$ 
We see in the right-hand side of the figure that the effect of $\delta_{h}$ is to localize the additional shape function $\psi^{\varepsilon}$ around the small obstacle. Indeed lemma [5.4 indicates that far away from the obstacle the additional shape function is well approximated by standard shape functions (since $\psi^{\varepsilon}$ is very smooth in this region), $\delta_{h}\left(\psi^{\varepsilon}\right)$ is close to 0 in this area. In some sense, the augmented numerical scheme (4.1) differs from the standard one (3.1) only in a region close to the small obstacle.

\subsection{Decomposition of the additional shape function}

In the next section we will have to know precisely the link between the additional shape function and the standard dicretization (the mesh and the usual shape functions). Note that the characteristic length of the additional shape function is $\varepsilon$, whereas the characteristic length of the mesh is $h$. An interesting question consists in finding how $\psi^{\varepsilon}$ is "seen" by the standard discretization. The decomposition (5.2), that we are going to establish, partly answers this question. It puts in evidence the dependency of $\mathcal{P}_{h}\left(\psi^{\varepsilon}\right)$ with respect to $\varepsilon$ and $h$. This will be useful in the proofs of Lemmas 6.3 and 6.4

The next study is based on the following idea: in the neighborhood of the small obstacle $\psi^{\varepsilon}(\mathbf{x}) \simeq \ln \left(\frac{|\mathbf{x}|}{\varepsilon}\right) \mathbf{1}_{|\mathbf{x}|>\varepsilon}$ and, modulo some terms related to the truncation, $\Delta \psi^{\varepsilon}=\delta_{\Gamma^{\varepsilon}} \Leftrightarrow \psi^{\varepsilon}=$ $\log * \delta_{\Gamma^{\varepsilon}}+C$, the constant term $C\left(=\ln \frac{1}{\varepsilon}\right)$ guarantying that $\left\langle\psi^{\varepsilon} ; \delta_{\Gamma^{\varepsilon}}\right\rangle=\int_{\Gamma^{\varepsilon}} \psi^{\varepsilon}=0$. What standard discretization cannot really reproduce here is $\delta_{\Gamma^{\varepsilon}}$; for two reasons: this is a singular object, and it is concentrated on a region with a diameter smaller than $h$, the characteristic length of the mesh.

Our approach consists in introducing a discrete version of $\delta_{\Gamma^{\varepsilon}}$. From this "numerical Dirac" we will deduce a discrete version of $\psi^{\varepsilon}$ that we will call regularized potential.

\subsubsection{Numerical Dirac Function}

We start from the equation defining $\delta_{\Gamma^{\varepsilon}}$. More precisely,

$$
\delta_{\Gamma^{\varepsilon}} \in \mathcal{D}^{\prime}\left(\mathbb{R}^{2}\right) \quad \text { and } \quad\left\langle\delta_{\Gamma^{\varepsilon}} ; v\right\rangle=\int_{\Gamma^{\varepsilon}} v, \quad \forall v \in \mathcal{D}\left(\mathbb{R}^{2}\right) .
$$

We propose discrete versions of these equations,

$$
g \in L^{2}\left(\mathbb{R}^{2}\right) \quad \text { and } \quad\left\langle g ; v^{h}\right\rangle=\int_{\Gamma^{\varepsilon}} v^{h}, \quad \forall v^{h} \in V^{h} .
$$

In addition we impose that supp $g$ have a support contained in a finite number of cells of the mesh around the small obstacle. Consider the sets $\mathcal{T}_{0}^{\varepsilon, h}=\left\{K \in \mathcal{T}_{h}\right.$ s.t $K$ has a vertex $\left.a_{i} \in \Omega_{\varepsilon}^{i}\right\}$ and $\mathcal{I}_{0}^{\varepsilon, h}=\left\{i \in \mathcal{I}\right.$ s.t $a_{i} \in K$ for some $\left.K \in \mathcal{T}_{0}^{\varepsilon, h}\right\}$. Define $\mathcal{O}_{\varepsilon, h}=\bigcup_{K \in \mathcal{T}_{0}^{\varepsilon, h}} K$. We also introduce a function $g \in L^{2}\left(\Omega_{R}\right)$ such that

$$
g(\mathbf{x})=\sum_{i \in \mathcal{I}_{0}} g_{i} \varphi_{i}^{h}(\mathbf{x}) \quad \text { if } \mathbf{x} \in \mathcal{O}_{\varepsilon, h} \quad \text { and } \quad g(\mathbf{x})=0 \quad \text { and } \mathbf{x} \in \Omega_{R} \backslash \mathcal{O}_{\varepsilon, h} .
$$

Note that $I_{0}^{\varepsilon, h}=\operatorname{card}\left(\mathcal{I}_{0}^{\varepsilon, h}\right)$ remains bounded when both $\varepsilon$ and $h$ go to 0 , and this implies that the sum above is always well defined. Indeed if we denote $\mathcal{A}\left(\mathcal{O}_{\varepsilon, h}\right)$ the area of $\mathcal{O}_{\varepsilon, h}$, it is easy to prove that there exists a non zero integer $\nu \in \mathbb{N}$ such that $\operatorname{card}\left(\mathcal{I}_{0}^{\varepsilon, h}\right) \leqslant \nu \operatorname{card}\left(\mathcal{T}_{0}^{\varepsilon, h}\right)(\nu=\operatorname{number}$ of degrees of freedom per element). Using assumptions $\mathbf{H} 1$ and $\mathbf{H} 2$, we get

$$
\begin{aligned}
\operatorname{card}\left(\mathcal{T}_{0}^{\varepsilon, h}\right) \pi \rho(h)^{2} \leqslant \mathcal{A}\left(\mathcal{O}_{\varepsilon, h}\right) \leqslant \pi(\varepsilon+h)^{2} & \Rightarrow \operatorname{card}\left(\mathcal{I}_{0}^{\varepsilon, h}\right) \frac{h^{2} \sigma_{-}^{2}}{\nu} \leqslant(1+\kappa)^{2} h^{2} \\
& \Rightarrow \operatorname{card}\left(\mathcal{I}_{0}^{\varepsilon, h}\right) \leqslant \frac{\nu}{\sigma_{-}^{2}}(1+\kappa)^{2} .
\end{aligned}
$$




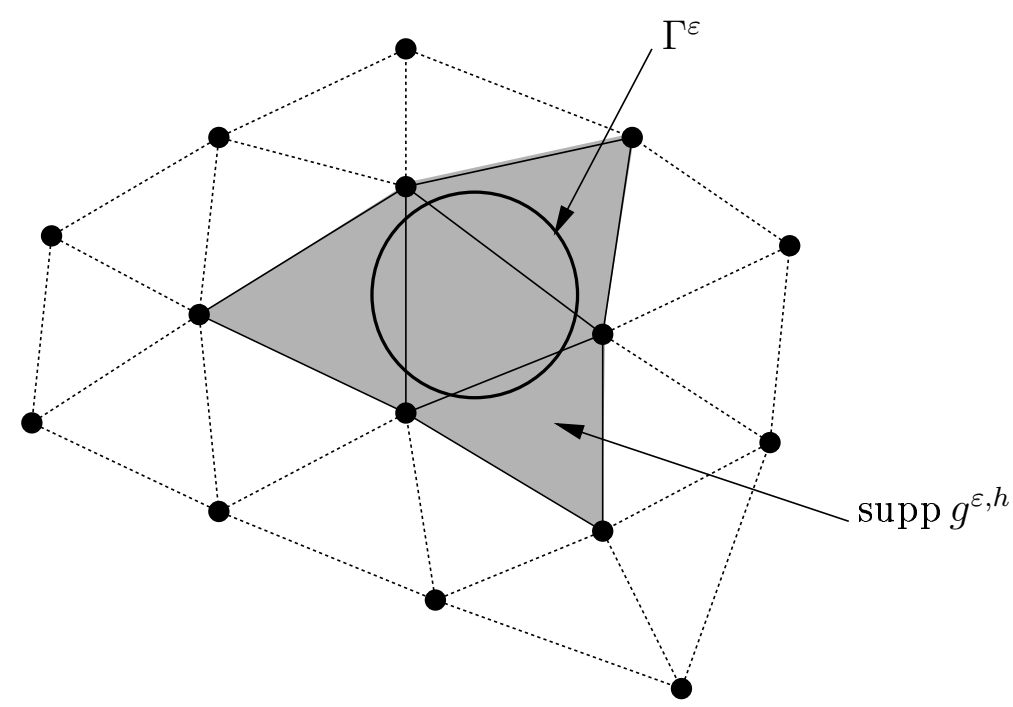

Figure 10: Support of the function $g$

Note also that for any $i \in \mathcal{I} \backslash \mathcal{I}_{0}^{\varepsilon, h}$, supp $\varphi_{i}^{h} \cap \operatorname{supp} g=\emptyset$. We choose coefficients $g_{i}$ for the relations $j=1 \cdots I, \quad \int_{\Omega_{R}} g \varphi_{j}=\int_{0}^{2 \pi} \varphi_{j}(\varepsilon, \theta) d \theta$ to be satisfied. Satisfying these conditions is equivalent to solving a linear system with the $g_{i}$ 's as unknowns,

$$
\mathbb{M}_{0}^{\varepsilon, h}\left[\begin{array}{l}
g_{1} \\
\vdots \\
g_{I_{0}^{\varepsilon, h}}
\end{array}\right]=\left[\begin{array}{l}
\int_{0}^{2 \pi} \varphi_{1}(\varepsilon, \theta) d \theta \\
\vdots \\
\int_{0}^{2 \pi} \varphi_{I_{0}^{\varepsilon, h}}(\varepsilon, \theta) d \theta
\end{array}\right] \quad \text { where } \quad\left(\mathbb{M}_{0}^{\varepsilon, h}\right)_{i, j}=\int_{\mathcal{O}_{\varepsilon, h}} \varphi_{i} \varphi_{j} \quad i, j=1 \ldots I_{0}^{\varepsilon, h}
$$

Arguments based on elementary geometry show that $\left|\int_{0}^{2 \pi} \varphi_{I_{0}^{\varepsilon, h}}(\varepsilon, \theta) d \theta\right| \leqslant 2 \pi \widetilde{\sigma}_{+}$. Moreover using the previous linear system defining $g$, it is possible to provide a bound for its $L^{2}$ norm. Indeed, $\|g\|_{L^{2}\left(\Omega_{R}\right)}^{2}=\sum_{i \in \mathcal{I}_{0}^{\varepsilon, h}} \int_{0}^{2 \pi} \varphi_{I_{0}^{\varepsilon, h}}(\varepsilon, \theta) d \theta g_{i}$. Moreover, using an homothety with ratio $h$ and center 0 in order to normalize the support of $g$, it is possible to prove the existence of some constant $\kappa>0$ such that $\|g\|_{L^{2}\left(\Omega_{R}\right)} \geqslant \kappa h\left(\sum_{i}\left|g_{i}\right|^{2}\right)^{1 / 2}$. We then obtain $\|g\|_{L^{2}\left(\Omega_{R}\right)}^{2} \leqslant$ $2 \pi \widetilde{\sigma}_{+}\left(I_{0}^{\varepsilon, h}\right)^{1 / 2}\left(\sum_{i}\left|g_{i}\right|^{2}\right)^{1 / 2} \leqslant \kappa 2 \pi \widetilde{\sigma}_{+}\left(I_{0}^{\varepsilon, h}\right)^{1 / 2}\|g\|_{L^{2}\left(\Omega_{R}\right)} / h$. Finally, this leads to the existence of a constant $\kappa>0$ independent of $h$ such that

$$
\|g\|_{L^{2}\left(\Omega_{R}\right)} \leqslant \kappa / h .
$$

Remark: Note that rigorously $g$ does depend on $\varepsilon$ and $h$. However, according to intuitive arguments, its dependancy with respect to $\varepsilon$ is very weak. For simplicity sake, we omit to write $g$ with $\varepsilon, h$ as index.

\subsubsection{Regularized Potential}

Consider $G(\mathbf{x}-\mathbf{y})=\frac{1}{2 \pi} \ln |\mathbf{x}-\mathbf{y}|$. Note that

$$
\psi^{\varepsilon}=\left(G * \delta_{\Gamma^{\varepsilon}}-\frac{1}{2 \pi}<\delta_{\Gamma^{\varepsilon}} ; G * \delta_{\Gamma^{\varepsilon}}>\right) \chi_{0} .
$$

We introduce a function similar to $\psi^{\varepsilon}$,

$$
\psi^{h}=\left(G * g-\frac{1}{2 \pi} \int_{\Omega_{R}} g G * g\right) \chi_{0} .
$$


Note that $G * g$ is solution to the problem

$$
G * g \in H^{1}\left(\Omega_{R}\right) \quad \text { and } \quad \int_{\Omega_{R}} \nabla(G * g) \cdot \nabla v=-\int_{\Omega_{R}} g v, \quad \forall v \in H_{0}^{1}\left(\Omega_{R}\right) .
$$

In particular, according to the construction of $g$,

$$
\int_{\Omega_{R}} \nabla(G * g) \cdot \nabla v_{h}=-\int_{\Omega_{R}} g v_{h}=-\int_{0}^{2 \pi} v_{h}(\varepsilon, \theta) d \theta, \quad \forall v \in V^{h}
$$

Let us compare $\psi^{\varepsilon}$ and $\psi^{h}$. Straightforward calculus provides

$$
\begin{aligned}
a\left(\psi^{\varepsilon}, v_{h}\right)= & \int_{\Omega_{R}} \nabla \psi^{\varepsilon} \cdot \nabla v_{h}-k^{2} \psi^{\varepsilon} v_{h} \\
= & -\int_{0}^{2 \pi} v_{h}(\varepsilon, \theta) d \theta-\int_{\Omega_{R} \backslash \Omega_{\varepsilon}^{i}} \Delta \psi^{\varepsilon} v_{h}-k^{2} \int_{\Omega_{R}} \psi^{\varepsilon} v_{h} \\
= & -\int_{0}^{2 \pi} v_{h}(\varepsilon, \theta) d \theta-2 \int_{\Omega_{R}} \nabla(\ln |\mathbf{x}|) \cdot \nabla \chi_{0} v_{h}-\int_{\Omega_{R} \backslash \Omega_{\varepsilon}^{i}} \ln \frac{|\mathbf{x}|}{\varepsilon}\left(\Delta \chi_{0}+k^{2} \chi_{0}\right) v_{h} \\
a\left(\psi^{h}, v_{h}\right)= & \int_{\Omega_{R}} \nabla \psi^{h} \cdot \nabla v_{h}-k^{2} \psi^{h} v_{h} \\
= & -\int_{\Omega_{R} \backslash \Omega_{\varepsilon}^{i}} \Delta \psi^{h} v_{h}-k^{2} \int_{\Omega_{R}} \psi^{h} v_{h} \\
= & -\int_{\Omega_{R}} g v_{h}-2 \int_{\Omega_{R}} \nabla(G * g) \cdot \nabla \chi_{0} v_{h} \\
& -\int_{\Omega_{R}}\left(G * g-\frac{1}{2 \pi} \int_{\Omega_{R}} g G * g\right)\left(\Delta \chi_{0}+k^{2} \chi_{0}\right) v_{h} .
\end{aligned}
$$

Taking the difference of these two equalities, we are led to

$$
\begin{aligned}
a\left(\psi^{\varepsilon}-\psi^{h}, v_{h}\right)= & -2 \int_{\Omega_{R}} \nabla(\ln |\mathbf{x}|-G * g) \cdot \nabla \chi_{0} v_{h} \\
& -\left(\ln \frac{1}{\varepsilon}+\frac{1}{2 \pi} \int_{\Omega_{R}} g G * g\right) \int_{\Omega_{R}}\left(\Delta \chi_{0}+k^{2} \chi_{0}\right) v_{h} \\
& -\int_{\Omega_{R}}(\ln |\mathbf{x}|-G * g)\left(\Delta \chi_{0}+k^{2} \chi_{0}\right) v_{h} .
\end{aligned}
$$

Define also $u_{1}, u_{2} \in H^{1}\left(\Omega_{R}\right)$ and $t(h) \in \mathbb{C}$ by

$$
\begin{aligned}
& a\left(u_{1}, v\right)=-\int_{\Omega_{R}}(\ln |\mathbf{x}|-G * g)\left(\Delta \chi_{0}+k^{2} \chi_{0}\right) v, \quad \forall v \in H^{1}\left(\Omega_{R}\right) \\
& a\left(u_{2}, v\right)=-\int_{\Omega_{R}} 2 \nabla(\ln |\mathbf{x}|-G * g) \cdot \nabla \chi_{0} v, \quad \forall v \in H^{1}\left(\Omega_{R}\right) \\
& t(h)=-\ln h+\frac{1}{2 \pi} \int_{\Omega_{R}} g G * g=\frac{1}{4 \pi^{2}} \int_{\Omega_{R}} \int_{\Omega_{R}} g(\mathbf{x}) g(\mathbf{y}) \ln \frac{|\mathbf{x}-\mathbf{y}|}{h} d \mathbf{x} d \mathbf{y} .
\end{aligned}
$$

Note that $\left\|u_{i}\right\|_{H^{2}\left(\Omega_{R}\right)} \rightarrow 0, i=1,2$ when $h$ goes to 0 (since $\|\ln |\mathbf{x}|-G * g\|_{L^{2}\left(\Omega_{R}\right)} \rightarrow 0$ ). Moreover the function $t(h)$ is bounded when $h$ goes to 0 as we can see using the estimate on the $L^{2}$ norm of $g$ that we provided before. According to Sobolev injection theorems, we conclude that $\left\|u_{i}\right\|_{L^{\infty}\left(\Omega_{R}\right)} \rightarrow$ 0 . Since the variational problem associated with $a$ is well posed, we obtain

$$
\psi^{\varepsilon}=\psi^{h}+\left(\ln \frac{h}{\varepsilon}+t(h)\right) \chi_{0}+u_{1}+u_{2} .
$$

According to the linearity of $\mathcal{P}_{h}$, there also holds

$$
\mathcal{P}_{h}\left(\psi^{\varepsilon}\right)=\mathcal{P}_{h}\left(\psi^{h}\right)+\left(\ln \frac{h}{\varepsilon}+t(h)\right) \mathcal{P}_{h}\left(\chi_{0}\right)+\mathcal{P}_{h}\left(u_{1}\right)+\mathcal{P}_{h}\left(u_{2}\right) .
$$


On the basis of Decomposition (5.2), we are now able to prove Lemma [5.1 that will be useful in Section [5.

Lemma 5.1 There exists a constant $\kappa>0$ independent of $h$ and $\varepsilon$, such that

$$
\left\|\delta_{h}\left(\psi^{\varepsilon}\right)\right\|_{H^{1}\left(\Omega_{R}\right)} \leqslant \kappa\left(1+h^{q} \ln \frac{h}{\varepsilon}\right) .
$$

\section{Proof:}

By linearity of $\delta_{h}, \delta_{h}\left(\psi^{\varepsilon}\right)=\delta_{h}\left(\psi^{h}\right)+\left(\ln \frac{h}{\varepsilon}+t(h)\right) \delta_{h}\left(\chi_{0}\right)+\delta_{h}\left(u_{1}\right)+\delta_{h}\left(u_{2}\right)$. We have to provide a bound in $H^{1}$ norm for each term of the previous expression Using standard estimates for finite elements and elliptic regularity, we obtain

$$
\begin{aligned}
& \left\|\delta_{h}\left(u_{1}\right)\right\|_{H^{1}\left(\Omega_{R}\right)} \leqslant \kappa h\left\|u_{1}\right\|_{H^{2}\left(\Omega_{R}\right)} \leqslant \kappa h\left\|(\ln |\mathbf{x}|-G * g)\left(\Delta \chi_{0}+k^{2} \chi_{0}\right)\right\|_{L^{2}\left(\Omega_{R}\right)} \leqslant \kappa \\
& \left\|\delta_{h}\left(u_{2}\right)\right\|_{H^{1}\left(\Omega_{R}\right)} \leqslant \kappa h\left\|u_{2}\right\|_{H^{2}\left(\Omega_{R}\right)} \leqslant \kappa h\left\|2 \nabla(\ln |\mathbf{x}|-G * g) \cdot \nabla \chi_{0}\right\|_{L^{2}\left(\Omega_{R}\right)} \leqslant \kappa .
\end{aligned}
$$

Moreover as the cut-off function is very smooth, the corresponding estimate does not raise any problem,

$$
\left\|\delta_{h}\left(\chi_{0}\right)\right\|_{H^{1}\left(\Omega_{R}\right)} \leqslant \kappa\left\|\chi_{0}\right\|_{H^{q+1}\left(\Omega_{R}\right)} h^{q} .
$$

Finally, concerning the regularized potential, we can write

$$
\left\|\delta_{h}\left(\psi^{h}\right)\right\|_{H^{1}\left(\Omega_{R}\right)} \leqslant \kappa h\left\|\psi^{h}\right\|_{H^{2}\left(\Omega_{R}\right)} \leqslant \kappa h\|G * g\|_{H^{2}\left(\Omega_{R}\right)} \leqslant \kappa h\|g\|_{L^{2}\left(\Omega_{R}\right)} \leqslant \kappa
$$

Note however that $h\|g\|_{L^{2}\left(\Omega_{R}\right)}$ does not go a priori to 0 . Gathering all the inequalities above, we obtain the desired result.

\section{The Holland model}

\subsection{Presentation of the Holland Model}

Now we will come to a model often used in practice: Holland type schemes, [8]. Let us define a bilinear form $c: Q_{\mathrm{ap}} \times Q_{\mathrm{ap}} \rightarrow \mathbb{C}$ by

$$
c(p, q)=\breve{p} \breve{q} .
$$

This introduces a coupling between Lagrange multipliers. On the basis of the ideas presented in [4, we will call scalar Holland scheme a discrete formulation of the form

$$
\left\{\begin{array}{l}
\text { Find }\left(u_{\mathbf{h}}^{\varepsilon, h}, p_{\mathbf{h}}^{\varepsilon, h}\right) \in V^{h} \times Q_{\text {ap }}^{h} \text { such that } \\
a\left(u_{\mathbf{h}}^{\varepsilon, h}, v\right)+b^{\varepsilon}\left(v, p_{\mathbf{h}}^{\varepsilon, h}\right)=\langle f, v\rangle_{V^{h}, V^{h^{\prime}}} \quad \forall v \in V^{h} \\
b^{\varepsilon}\left(u_{\mathbf{h}}^{\varepsilon, h}, q\right)-L_{\mathbf{h}}^{\varepsilon, h} c\left(p_{\mathbf{h}}^{\varepsilon, h}, q\right)=0 \quad \forall q \in Q_{\mathrm{ap}}^{h} .
\end{array}\right.
$$

Here the constant $L_{\mathbf{h}}^{\varepsilon, h}$, sometimes called lineic inductance, is not a priori known. In practice it is chosen on the basis of empirical considerations. This type of scheme was proposed by Holland in order to solve Maxwell equations in the exterior of perfectly conducting thin wires in [ 8$]$. However this article proposes a modification of a finite difference scheme in a Cartesian grid with wires aligned with the axis of the grid. Collino and Millot in 4 proposed a continuous setting, that enables to recover the Holland scheme in the case of a finite difference discretization, but also 
leads to a generalization for finite elements. They also obtain a simple theoretical expression of the lineic inductance in the case of a wire aligned with the axis of a Cartesian grid. In the case of an arbitrary mesh, and for a wire arbitrarily oriented, the expression of the lineic inductance has remained an open question though some empirical formulas have been proposed. Experience has shown that the Holland scheme possesses several interesting characteristics:

- The differences between Holland scheme and a classical scheme are localized around the wires.

- It is not difficult to implement it.

- If the lineic inductance is well chosen, the Holland model provides precise results for the field away from the wires and for the current flowing across the wire.

However there also arise several drawbacks:

- No a priori formula is available for the lineic inductance, one has to choose it in accordance with many experiments.

- To the best of our knowledge, there exists no theoretical justification of the Holland model: in particular there exists no consistency result.

An interesting result though has been proposed in [4] concerning the lineic inductance. Suppose the mesh is Cartesian in a neighborhood of the small obstacle, and suppose this obstacle to be a disk of radius $\varepsilon$ centered around a vertex of the mesh. This is the situation represented in the figure below.

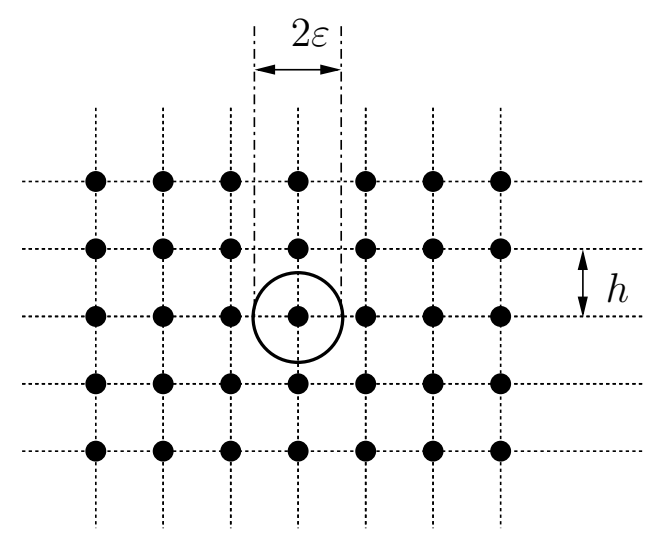

Figure 11: Cartesian mesh around the small obstacle

In this situation, Collino and Millot derived an approximate expression for the lineic inductance,

$$
L_{\mathbf{h}}^{\varepsilon, h} \simeq \ln \left(\frac{h}{\varepsilon}\right)+\kappa_{\mathbf{h}}
$$

where $\kappa_{\mathbf{h}}$ is independent of $h$ and $\varepsilon$ (and also independent of the wave number $k$ !). We propose to recover the Holland scheme, and also to justify this formula for the lineic inductance in the case of an arbitrary 2-D mesh. 


\subsection{Relation between augmented Galerkin scheme and Holland scheme}

In this paragraph we establish a link between the Holland scheme (6.1) and the augmented Galerkin scheme (4.1). This will lead us to a theoretical analysis of the Holland model. Let us get back to decomposition (5.1) in a regular part and a singular part that was proposed at the end of the previous section.

$$
u^{\varepsilon, h}=u_{\mathbf{r}}^{\varepsilon, h}+p_{\mathbf{s}}^{\varepsilon, h} \delta_{h}\left(\psi^{\varepsilon}\right) .
$$

Formulation (4.1) is strictly equivalent to

$$
\left\{\begin{array}{l}
\text { Find } \quad\left(u_{\mathbf{r}}^{\varepsilon, h}, p_{\mathbf{s}}^{\varepsilon, h}, p^{\varepsilon, h}\right) \in V^{h} \times \mathbb{C} \times Q_{h} \quad \text { such that } \\
a\left(u_{\mathbf{r}}^{\varepsilon, h}, w_{h}\right)+b^{\varepsilon}\left(w_{h}, p^{\varepsilon, h}\right)=\left\langle f, w_{h}\right\rangle_{V, V^{\prime}} \quad \forall w_{h} \in V^{h} \\
p_{\mathbf{s}}^{\varepsilon, h} a\left(\delta_{h}\left(\psi^{\varepsilon}\right), \delta_{h}\left(\psi^{\varepsilon}\right)\right)+b^{\varepsilon}\left(\delta_{h}\left(\psi^{\varepsilon}\right), p^{\varepsilon, h}\right)=\left\langle f, \delta_{h}\left(\psi^{\varepsilon}\right)\right\rangle_{V, V^{\prime}} \\
b^{\varepsilon}\left(u_{\mathbf{r}}^{\varepsilon, h}, q_{h}\right)+p_{\mathbf{s}}^{\varepsilon, h} b^{\varepsilon}\left(\delta_{h}\left(\psi^{\varepsilon}\right), q_{h}\right)=0 \quad \forall q_{h} \in Q_{h} .
\end{array}\right.
$$

Using the second equation of this system, we obtain

$$
p_{\mathbf{s}}^{\varepsilon, h}=-\breve{p}^{\varepsilon, h} \frac{\breve{b}^{\varepsilon}\left(\delta_{h}\left(\psi^{\varepsilon}\right)\right)}{a\left(\delta_{h}\left(\psi^{\varepsilon}\right), \delta_{h}\left(\psi^{\varepsilon}\right)\right)}+\frac{\left\langle f, \delta_{h}\left(\psi^{\varepsilon}\right)\right\rangle_{V, V^{\prime}}}{a\left(\delta_{h}\left(\psi^{\varepsilon}\right), \delta_{h}\left(\psi^{\varepsilon}\right)\right)}
$$

Putting this equality in (6.2) yields

$$
\left\{\begin{array}{l}
\text { Find } \quad\left(u_{\mathbf{r}}^{\varepsilon, h}, p^{\varepsilon, h}\right) \in V^{h} \times Q_{h} \quad \text { such that } \\
a\left(u_{\mathbf{r}}^{\varepsilon, h}, w_{h}\right)+b^{\varepsilon}\left(w_{h}, p^{\varepsilon, h}\right)=\left\langle f, w_{h}\right\rangle_{V, V^{\prime}} \quad \forall w_{h} \in V^{h} \\
b^{\varepsilon}\left(u_{\mathbf{r}}^{\varepsilon, h}, q_{h}\right)-\frac{\breve{b}^{\varepsilon}\left(\delta_{h}\left(\psi^{\varepsilon}\right)\right)^{2}}{a\left(\delta_{h}\left(\psi^{\varepsilon}\right), \delta_{h}\left(\psi^{\varepsilon}\right)\right)} c\left(p^{\varepsilon, h}, q_{h}\right)=\left\langle d^{\varepsilon, h}, q_{h}\right\rangle_{Q^{\prime}, Q} \quad \forall q_{h} \in Q_{h} .
\end{array}\right.
$$

There appears a new term in the right-hand side that is defined as follows

$$
\left\langle d^{\varepsilon, h}, q_{h}\right\rangle_{Q^{\prime}, Q}=-\breve{q}_{h} \frac{\breve{b}^{\varepsilon}\left(\delta_{h}\left(\psi^{\varepsilon}\right)\right)\left\langle f, \delta_{h}\left(\psi^{\varepsilon}\right)\right\rangle_{V, V^{\prime}}}{a\left(\delta_{h}\left(\psi^{\varepsilon}\right), \delta_{h}\left(\psi^{\varepsilon}\right)\right)}
$$

Note that Formulation (6.4) is very close to 6.1). Indeed if we choose

$$
L_{\mathbf{h}}^{\varepsilon, h}=\frac{\breve{b}^{\varepsilon}\left(\delta_{h}\left(\psi^{\varepsilon}\right)\right)^{2}}{a\left(\delta_{h}\left(\psi^{\varepsilon}\right), \delta_{h}\left(\psi^{\varepsilon}\right)\right)}=-\breve{b}^{\varepsilon}\left(\delta_{h}\left(\psi^{\varepsilon}\right)\right) \times \frac{-\breve{b}^{\varepsilon}\left(\delta_{h}\left(\psi^{\varepsilon}\right)\right)}{a\left(\delta_{h}\left(\psi^{\varepsilon}\right), \delta_{h}\left(\psi^{\varepsilon}\right)\right)}
$$

the only difference between these two formulations is the additional source term $d^{\varepsilon, h}$ in (6.4). However this term is negligible in the following sense:

\section{Lemma 6.1}

There exists $\kappa>0$ independent of $h$ such that $\left|d^{\varepsilon, h}\right| \leqslant \kappa h^{q}$

\section{Proof:}

We reinterpret $\left\langle f, \delta_{h}\left(\psi^{\varepsilon}\right)\right\rangle_{V, V^{\prime}}=-\int_{\Omega_{R}} f \delta_{h}\left(\psi^{\varepsilon}\right)$. There exists an open set $\mathcal{O} \subset \Omega_{R} \backslash \overline{\operatorname{supp} \psi^{\varepsilon}}$ such that $\operatorname{supp} f \subset \mathcal{O}$. Using corollary [5.1] and lemma [5.2] we obtain

$$
\left|\frac{\left\langle f, \delta_{h}\left(\psi^{\varepsilon}\right)\right\rangle_{V, V^{\prime}}}{a\left(\delta_{h}\left(\psi^{\varepsilon}\right), \delta_{h}\left(\psi^{\varepsilon}\right)\right)}\right| \leqslant \kappa\|f\|_{L^{2}\left(\Omega_{R}\right)}\left\|\delta_{h}\left(\psi^{\varepsilon}\right)\right\|_{L^{2}(\mathcal{O})} \leqslant \kappa\|f\|_{L^{2}\left(\Omega_{R}\right)} h^{q+1}\left(1+\ln \frac{h}{\varepsilon}\right) .
$$


Moreover note that, according to Lemmas [5.1] and 2.5] there holds

$$
\left|\breve{b}^{\varepsilon}\left(\delta_{h}\left(\psi^{\varepsilon}\right)\right)\right| \leqslant \kappa \sqrt{\ln 1 / \varepsilon}\left(1+\ln \frac{h}{\varepsilon}\right) .
$$

Thus we obtain

$$
\left|d^{\varepsilon, h}\right|=\left|\frac{\breve{b^{\varepsilon}}\left(\delta_{h}\left(\psi^{\varepsilon}\right)\right)\left\langle f, \delta_{h}\left(\psi^{\varepsilon}\right)\right\rangle_{V, V^{\prime}}}{a\left(\delta_{h}\left(\psi^{\varepsilon}\right), \delta_{h}\left(\psi^{\varepsilon}\right)\right)}\right| \leqslant \kappa\|f\|_{L^{2}\left(\Omega_{R}\right)} h^{q}\left(h \sqrt{\ln 1 / \varepsilon}\left(1+\ln \frac{h}{\varepsilon}\right)^{2}\right) .
$$

Applying assumption H3, we obtain the existence of a constant $\kappa>0$ independent of $h$ such that $\left|h \sqrt{\ln 1 / \varepsilon}\left(1+\ln \frac{h}{\varepsilon}\right)^{2}\right| \leqslant \kappa$. This leads us to the conclusion.

According to this result it is somehow possible to identify $u_{\mathbf{h}}^{\varepsilon, h}$ and $u_{\mathbf{r}}^{\varepsilon, h}$. This also leads to the theoretical expression (6.5) providing the Holland parameter. The next lemma confirms that $u_{\mathbf{h}}^{\varepsilon, h}$ is close to $u_{\mathbf{r}}^{\varepsilon, h}$.

\section{Lemma 6.2}

There exists a constant $\kappa>0$ independent of $\varepsilon$ and $h$, such that

$$
\left\|u_{\mathbf{h}}^{\varepsilon, h}-u_{\mathbf{r}}^{\varepsilon, h}\right\|_{H^{1}\left(\Omega_{R}\right)}+\left|p^{\varepsilon, h}-p_{\mathbf{h}}^{\varepsilon, h}\right| \leqslant \kappa h^{q}
$$

\section{Proof:}

The couple $\left(u_{\mathbf{r}}^{\varepsilon, h}-u_{\mathbf{h}}^{\varepsilon, h}, p^{\varepsilon, h}-p_{\mathbf{h}}^{\varepsilon, h}\right) \in V^{h} \times Q_{h}$ and satisfies

$$
\begin{aligned}
& a\left(u_{\mathbf{r}}^{\varepsilon, h}, w_{h}\right)+b^{\varepsilon}\left(w_{h}, p^{\varepsilon, h}\right)=0, \quad \forall w_{h} \in V^{h} \\
& b^{\varepsilon}\left(u_{\mathbf{r}}^{\varepsilon, h}, q_{h}\right)-L_{\mathbf{h}}^{\varepsilon, h} c\left(p^{\varepsilon, h}, q_{h}\right)=\left\langle d^{\varepsilon, h}, q_{h}\right\rangle_{Q^{\prime}, Q} \quad \forall q_{h} \in Q_{h} .
\end{aligned}
$$

Thus it is possible to apply the result of theorem $1.2 \S I I .1$ of [1] and use lemma 6.1 in order to obtain the existence $\kappa>0$ independent of $\varepsilon$ and $h$ such that

$$
\left\|u_{\mathbf{h}}^{\varepsilon, h}-u_{\mathbf{r}}^{\varepsilon, h}\right\|_{H^{1}\left(\Omega_{R}\right)}+\left|p^{\varepsilon, h}-p_{\mathbf{h}}^{\varepsilon, h}\right| \leqslant \kappa\left|d^{\varepsilon, h}\right| \leqslant \kappa h^{q} .
$$

\subsection{Theoritical Expression of the Lineic Inductance}

We obtained an explicit expression for the lineic inductance

$$
L_{\mathbf{h}}^{\varepsilon, h}=\frac{\breve{b}^{\varepsilon}\left(\delta_{h}\left(\psi^{\varepsilon}\right)\right)^{2}}{a\left(\delta_{h}\left(\psi^{\varepsilon}\right), \delta_{h}\left(\psi^{\varepsilon}\right)\right)}=-\breve{b}^{\varepsilon}\left(\delta_{h}\left(\psi^{\varepsilon}\right)\right) \times \frac{-\breve{b}^{\varepsilon}\left(\delta_{h}\left(\psi^{\varepsilon}\right)\right)}{a\left(\delta_{h}\left(\psi^{\varepsilon}\right), \delta_{h}\left(\psi^{\varepsilon}\right)\right)} .
$$

We will use this expression in order to prove some properties for the lineic inductance. This is the meaning of the next results. For this analysis, we will introduce a further assumption that will be supposed verified for the rest of this document.

H3 : There exists $\kappa>0$ and $\nu>0$ such that $\varepsilon(h) \geqslant \kappa h^{1+\nu}$.

This is a restrictive assumption. Note however that it is possible to suppose that $\nu$ is large, for example $\nu=10$. This implies the following bound: there exists $\kappa>0$ independent of $\varepsilon$ and $h$ 
such that $|\ln (h / \varepsilon)|<\kappa \ln (1 / h)$. Considering Lemmas 5.1 and 5.3 we obtain existence of $\kappa>0$ independent of $\varepsilon$ and $h$ such that

$$
\left\|\psi^{\varepsilon}\right\|_{H^{1}\left(\Omega_{R}\right)}<\kappa \quad \text { et }\left\|\psi^{\varepsilon}\right\|_{L^{2}\left(\Omega_{R}\right)}<\kappa h
$$

The lineic inductance can be split into two parts, as this appears in Formula 6.5. Lemma 6.3 yields some information about the first part while Lemma 6.4 deals with the second one. By the way, we will finally obtain an expression very close to the one pointed out in [4] for Cartesian grids.

Lemma 6.3 There exists a function $r(\varepsilon(h), h)$ bounded as $h \rightarrow 0$ such that

$$
-\breve{b}^{\varepsilon}\left(\delta_{h}\left(\psi^{\varepsilon}\right)\right)=\ln \left(\frac{h}{\varepsilon}\right)+r(\varepsilon, h)
$$

Proof: We start using the definition of $g$ and the decomposition in (5.2). As $\psi^{\varepsilon}=0$ on $\Gamma^{\varepsilon}$,

$$
\begin{aligned}
-\breve{b}^{\varepsilon}\left(\delta_{h}\left(\psi^{\varepsilon}\right)\right)= & \int_{0}^{2 \pi} \mathcal{P}_{h}\left(\psi^{\varepsilon}\right)(\varepsilon, \theta) d \theta=\int_{\Omega_{R}} \mathcal{P}_{h}\left(\psi^{\varepsilon}\right) g \\
= & \int_{\Omega_{R}} \mathcal{P}_{h}\left(\psi^{h}\right) g+\left(\ln \frac{h}{\varepsilon}+t(h)\right) \int_{\Omega_{R}} \mathcal{P}_{h}\left(\chi_{0}\right) g \\
& +\int_{\Omega_{R}} \mathcal{P}_{h}\left(u_{1}\right) g+\int_{\Omega_{R}} \mathcal{P}_{h}\left(u_{2}\right) g
\end{aligned}
$$

We will study each of the terms appearing in the right-hand side above. First, the second term can be rewritten in the following manner

$$
\begin{aligned}
\int_{\Omega_{R}} \mathcal{P}_{h}\left(\chi_{0}\right) g & =\int_{\Omega_{R}} \chi_{0} g+\int_{\Omega_{R}}\left(\mathcal{P}_{h}\left(\chi_{0}\right)-\chi_{0}\right) g \\
& =\int_{\Omega_{R}} g+\int_{\Omega_{R}}\left(\mathcal{P}_{h}\left(\chi_{0}\right)-\chi_{0}\right) g=2 \pi+\int_{\Omega_{R}}\left(\mathcal{P}_{h}\left(\chi_{0}\right)-\chi_{0}\right) g .
\end{aligned}
$$

Moreover the following estimate holds

$$
\left|\int_{\Omega_{R}}\left(\mathcal{P}_{h}\left(\chi_{0}\right)-\chi_{0}\right) g\right| \leqslant\|h g\|_{L^{2}\left(\Omega_{R}\right)} \frac{\left\|\mathcal{P}_{h}\left(\chi_{0}\right)-\chi_{0}\right\|_{L^{2}\left(\Omega_{R}\right)}}{h} \leqslant \kappa\left\|\chi_{0}\right\|_{H^{2}\left(\Omega_{R}\right)} h .
$$

Using assumption $\mathbf{H}$ 3, we obtain the existence of some $\kappa$ independent of $h$ such that

$$
\left|\ln \frac{h}{\varepsilon} \frac{1}{2 \pi} \int_{\Omega_{R}} \mathcal{P}_{h}\left(\chi_{0}\right) g-\ln \frac{h}{\varepsilon}\right| \leqslant \kappa .
$$

The third and fourth term can be decomposed in the following manner $(i=1,2)$

$$
\int_{\Omega_{R}} \mathcal{P}_{h}\left(u_{i}\right) g=\int_{\Omega_{R}} u_{i} g+\int_{\Omega_{R}}\left(\mathcal{P}_{h}\left(u_{i}\right)-u_{i}\right) g .
$$

Moreover we have the estimate

$$
\left|\int_{\Omega_{R}} u_{i} g\right| \leqslant\left\|u_{i}\right\|_{L^{\infty}\left(\Omega_{R}\right)}\|g\|_{L^{1}\left(\Omega_{R}\right)} \leqslant \kappa\left\|u_{i}\right\|_{L^{\infty}\left(\Omega_{R}\right)}\|h g\|_{L^{2}\left(\Omega_{R}\right)}
$$

and

$$
\left|\int_{\Omega_{R}}\left(\mathcal{P}_{h}\left(u_{i}\right)-u_{i}\right) g\right| \leqslant\|h g\|_{L^{2}\left(\Omega_{R}\right)} \frac{\left\|\mathcal{P}_{h}\left(u_{i}\right)-u_{i}\right\|_{L^{2}\left(\Omega_{R}\right)}}{h} \leqslant \kappa\left\|u_{i}\right\|_{H^{2}\left(\Omega_{R}\right)} h .
$$

Finally for the first term, $\int_{\Omega_{R}} \mathcal{P}_{h}\left(\psi^{h}\right) g=\int_{\Omega_{R}} \delta_{h}\left(\psi^{h}\right) g$ since $\int_{\Omega_{R}} \psi^{h} g=0$. Hence,

$$
\left|\int_{\Omega_{R}} \delta_{h}\left(\psi^{h}\right) g\right| \leqslant\left\|\delta_{h}\left(\psi^{h}\right)\right\|_{L^{2}\left(\Omega_{R}\right)}\|g\|_{L^{2}\left(\Omega_{R}\right)} \leqslant \kappa h^{2}\left\|\psi^{h}\right\|_{H^{2}\left(\Omega_{R}\right)}\|g\|_{L^{2}\left(\Omega_{R}\right)} \leqslant \kappa\left(h\|g\|_{L^{2}\left(\Omega_{R}\right)}\right)^{2}
$$


Using all the preceding result, we obtain the conclusion.

We will now analyze the second part of the lineic inductance. It is inherited from Equation 6.3. Moreover it is possible to provide a formal interpretation of this relation. Let us use qualitative arguments. Corollary (4.1) suggests that $u^{\varepsilon, h} \simeq \widetilde{u}^{\varepsilon}$. Moreover

$$
p^{\varepsilon, h} \simeq \widetilde{p}^{\varepsilon}=\left[\frac{\partial \widetilde{u}^{\varepsilon}}{\partial n}\right]_{\mid \Gamma^{\varepsilon}} \simeq\left[\frac{\partial u^{\varepsilon, h}}{\partial n}\right]_{\mid \Gamma^{\varepsilon}}=\underbrace{\left[\frac{\partial u_{\mathbf{r}}^{\varepsilon, h}}{\partial n}\right]_{\mid \Gamma^{\varepsilon}}}_{\simeq 0}+\left[\frac{\partial}{\partial n} p_{\mathbf{s}}^{\varepsilon, h} V_{0}^{\varepsilon}\right]_{\mid \Gamma^{\varepsilon}} \simeq p_{\mathbf{s}}^{\varepsilon, h} \frac{\partial V_{0}^{\varepsilon}}{\partial n} .
$$

This finally yields $\breve{p}^{\varepsilon, h} \simeq p_{\mathbf{s}}^{\varepsilon, h}$. Now look at relation 6.3. Remind that the second term of the right-hand side is small. In addition we can show that $1 / p^{\varepsilon, h}$ is bounded by something proportional to $\ln 1 / \varepsilon$ (i.e. $p^{\varepsilon, h} \neq 0$ ). then we obtain

$$
\frac{-\breve{b} \varepsilon\left(\delta_{h}\left(\psi^{\varepsilon}\right)\right)}{a\left(\delta_{h}\left(\psi^{\varepsilon}\right), \delta_{h}\left(\psi^{\varepsilon}\right)\right)} \simeq 1
$$

This intuition is confirmed by the next lemma. We also illustrate this with the numerical experiments presented in Figure 12

\section{Lemma 6.4}

$$
\frac{-\breve{b}^{\varepsilon}\left(\delta_{h}\left(\psi^{\varepsilon}\right)\right)}{a\left(\delta_{h}\left(\psi^{\varepsilon}\right), \delta_{h}\left(\psi^{\varepsilon}\right)\right)}=1+O(h \ln 1 / h) \quad \text { when } \quad h \rightarrow 0
$$

\section{Proof:}

Write

$$
\begin{aligned}
a\left(\delta_{h}\left(\psi^{\varepsilon}\right), \delta_{h}\left(\psi^{\varepsilon}\right)\right)=a\left(\psi^{\varepsilon}, \delta_{h}\left(\psi^{\varepsilon}\right)\right) & =\int_{0}^{2 \pi} \mathcal{P}_{h}\left(\psi^{\varepsilon}\right)(\varepsilon, \theta) d \theta-2 \int_{\Omega_{R}} \nabla(\ln |\mathbf{x}|) \cdot \nabla \chi_{0} \delta_{h}\left(\psi^{\varepsilon}\right) \\
& -\int_{\Omega_{R} \backslash \Omega_{\varepsilon}^{i}} \ln \frac{|\mathbf{x}|}{\varepsilon}\left(\Delta \chi_{0}+k^{2} \chi_{0}\right) \delta_{h}\left(\psi^{\varepsilon}\right)
\end{aligned}
$$

According to Lemma 5.2 there exists $\kappa>0$ such that $a\left(\delta_{h}\left(\psi^{\varepsilon}\right), \delta_{h}\left(\psi^{\varepsilon}\right)\right)>\kappa$. Dividing this by $a\left(\delta_{h}\left(\psi^{\varepsilon}\right), \delta_{h}\left(\psi^{\varepsilon}\right)\right)$, we obtain

$$
\frac{-\breve{b}^{\varepsilon}\left(\delta_{h}\left(\psi^{\varepsilon}\right)\right)}{a\left(\delta_{h}\left(\psi^{\varepsilon}\right), \delta_{h}\left(\psi^{\varepsilon}\right)\right)}=1+2 \int_{\Omega_{R}} \frac{\nabla(\ln |\mathbf{x}|) \cdot \nabla \chi_{0} \delta_{h}\left(\psi^{\varepsilon}\right)}{a\left(\delta_{h}\left(\psi^{\varepsilon}\right), \delta_{h}\left(\psi^{\varepsilon}\right)\right)}+\int_{\Omega_{R} \backslash \Omega_{\varepsilon}^{i}} \frac{\ln \frac{|\mathbf{x}|}{\varepsilon}\left(\Delta \chi_{0}+k^{2} \chi_{0}\right) \delta_{h}\left(\psi^{\varepsilon}\right)}{a\left(\delta_{h}\left(\psi^{\varepsilon}\right), \delta_{h}\left(\psi^{\varepsilon}\right)\right)} .
$$

On the one hand, there exists $\kappa>0$ independent of $\varepsilon$ and $h$ such that

$$
\left\|\nabla(\ln |\mathbf{x}|) \cdot \nabla \chi_{0}\right\|_{L^{2}\left(\Omega_{R}\right)} \leqslant \kappa \quad \text { et } \quad\left\|\ln \frac{|\mathbf{x}|}{\varepsilon}\left(\Delta \chi_{0}+k^{2} \chi_{0}\right)\right\|_{L^{2}\left(\Omega_{R} \backslash \Omega_{\varepsilon}^{i}\right)} \leqslant \kappa \ln 1 / h .
$$

On the second hand, Inequalities [6.7 along with the Cauchy-Schwartz inequality provide

$$
\left|\int_{\Omega_{R}} \nabla(\ln |\mathbf{x}|) \cdot \nabla \chi_{0} \delta_{h}\left(\psi^{\varepsilon}\right)\right| \leqslant \kappa h \quad \text { et }\left|\int_{\Omega_{R} \backslash \Omega_{\varepsilon}^{i}} \ln \frac{|\mathbf{x}|}{\varepsilon}\left(\Delta \chi_{0}+k^{2} \chi_{0}\right) \delta_{h}\left(\psi^{\varepsilon}\right)\right| \leqslant \kappa h \ln 1 / h
$$

This leads to the desired result.

By means of Lemmas 6.4 and 6.3, we have now described the two parts of the lineic inductance. We can deduce a substantial simplification of expression (6.5). 


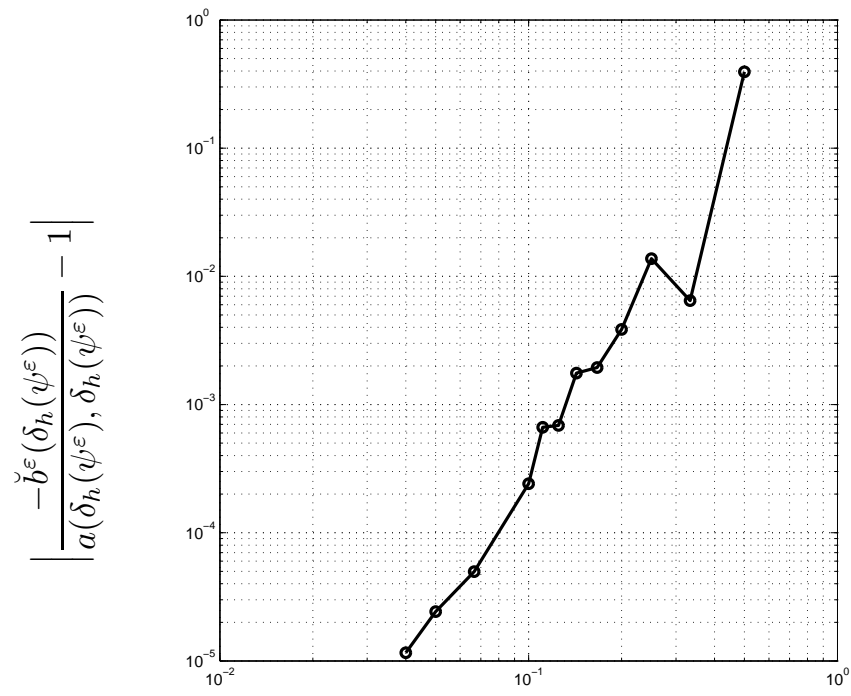

Thickness $\varepsilon / h=10^{-4}$

Finite elements of order 3

Nombre de points par longueur d'onde $h / \lambda$

Figure 12: Convergence of the ratio defined in Lemma 6.4

\section{Corollary 6.1}

$$
\left|L_{\mathbf{h}}^{\varepsilon, h}-b^{\varepsilon}\left(\delta_{h}\left(\psi^{\varepsilon}\right)\right)\right| \leqslant \kappa h \ln ^{2}(1 / h) .
$$

\section{Proof:}

Using assumption H3, one easily see that $\left|b^{\varepsilon}\left(\delta_{h}\left(\psi^{\varepsilon}\right)\right)\right| \leqslant \kappa \ln 1 / h$. Thus using lemmas 6.4 and 5.2 we obtain the desired result.

Provided that assumptions H1, H2 and $\mathbf{H 3}$ are satisfied, we can give the following approximate expression

$$
L_{\mathbf{h}}^{\varepsilon, h}=\ln \frac{h}{\varepsilon}+r(\varepsilon, h)+O\left(h \ln ^{2} 1 / h\right)
$$

To sum up, the Holland constant for our model problem a priori depends on several parameters: the mesh (its shape and its step $h$ ), the thickness of the wire $\varepsilon$, the wave number $k$ and the cut-off function $\chi$. In the next two paragraphs we will show that the Holland parameter

- weakly depends on the wave number $k$ (which allows us to consider a generalization to time dependent situations),

- strongly depends on the position of the wire in the mesh,

- weakly depends on the radius of the cut-off function $\chi$.

\section{Perspectives toward time dependent problems}

In addition to harmonic regimes, it is also possible to propose a Holland scheme for time dependent problems. In this case, the Holland scheme has the advantage to be stable under the usual CFL condition, which means that in this case the presence of the wire does not affect the stability. Along with the previous analysis appears a natural question: what has to be the appropriate value of the Holland parameter in time dependent schemes? This question seems quite difficult at first 
glance since the expression (6.5) does depend on the wave number $k$ via the bilinear form $a$. However numerical experiment show that actually the value of the expression (6.5) is nearly insensitive to variations of the frequency. In figure 13, we have represented (6.5) versus the frequency (more precisely versus the number of points per wavelength with a fixed step $h$ ), and we see that this dependency is very weak.

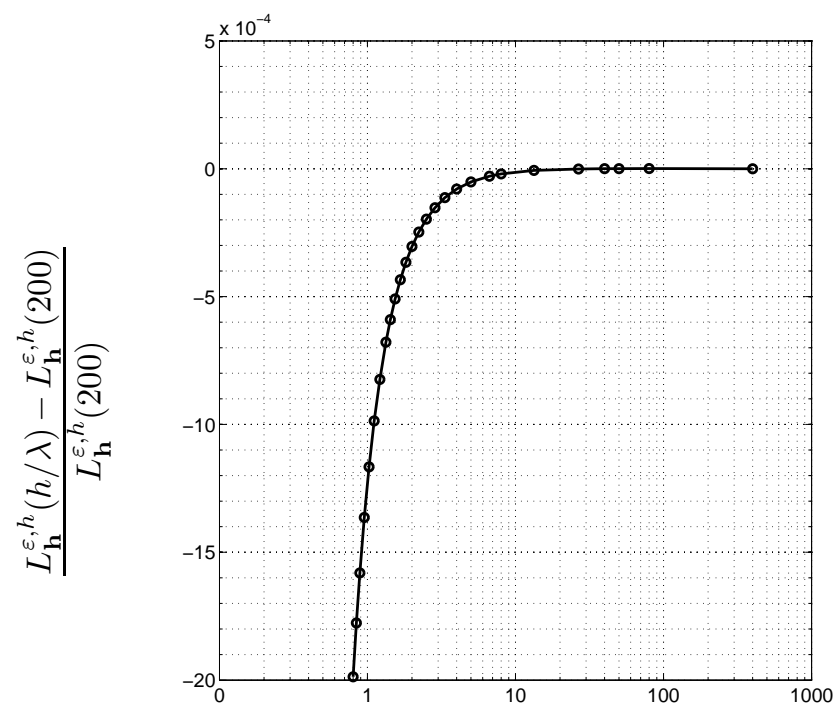

Thickness $\varepsilon / h=10^{-4}$

Finite elements of order 4 $L_{\mathbf{h}}^{\varepsilon, h}(200)=0.918$

Number of points per wavelength $\lambda / H$

Figure 13: Relative variation of the lineic inductance.

This suggests us to compute expression (6.5) for an electrostatic problem, or compute it for a coercive problem, and use the corresponding Holland parameter for the following semi-discretized scheme

$$
\begin{aligned}
& \text { Find }\left(u_{\mathbf{r}}^{\varepsilon, h}, p^{\varepsilon, h}\right) \in C^{1}\left([0, T], V^{h}\right) \times C^{1}\left([0, T], Q_{h}\right) \quad \text { such that } \\
& \left\{\frac{d^{2}}{d t^{2}} \int_{\Omega_{R}} u_{\mathbf{r}}^{\varepsilon, h}(t) w_{h}+\int_{\Omega_{R}} \nabla u_{\mathbf{r}}^{\varepsilon, h}(t) \cdot \nabla w_{h}+\int_{\Gamma_{R}} \frac{\partial u_{\mathbf{r}}^{\varepsilon, h}}{\partial t}(t) w_{h}+b^{\varepsilon}\left(w_{h}, \frac{\partial p^{\varepsilon, h}}{\partial t}\right)\right. \\
& =\left\langle f, w_{h}\right\rangle_{V, V^{\prime}}, \forall w_{h} \in V^{h} \\
& b^{\varepsilon}\left(u_{\mathbf{r}}^{\varepsilon, h}, q_{h}\right)-L_{\mathbf{h}}^{\varepsilon, h} c\left(\frac{\partial p^{\varepsilon, h}}{\partial t}, q_{h}\right)=0 \quad \forall q_{h} \in Q_{h} .
\end{aligned}
$$

The study of the properties of such a scheme will be the subject of a forthcoming work.

\section{Dependency with respect to the location of the obstacle in the mesh}

Whereas the lineic inductance nearly does not depend on frequency, we will see that it really depends on the location of the wire inside the mesh. We illustrate this in Figure 14 

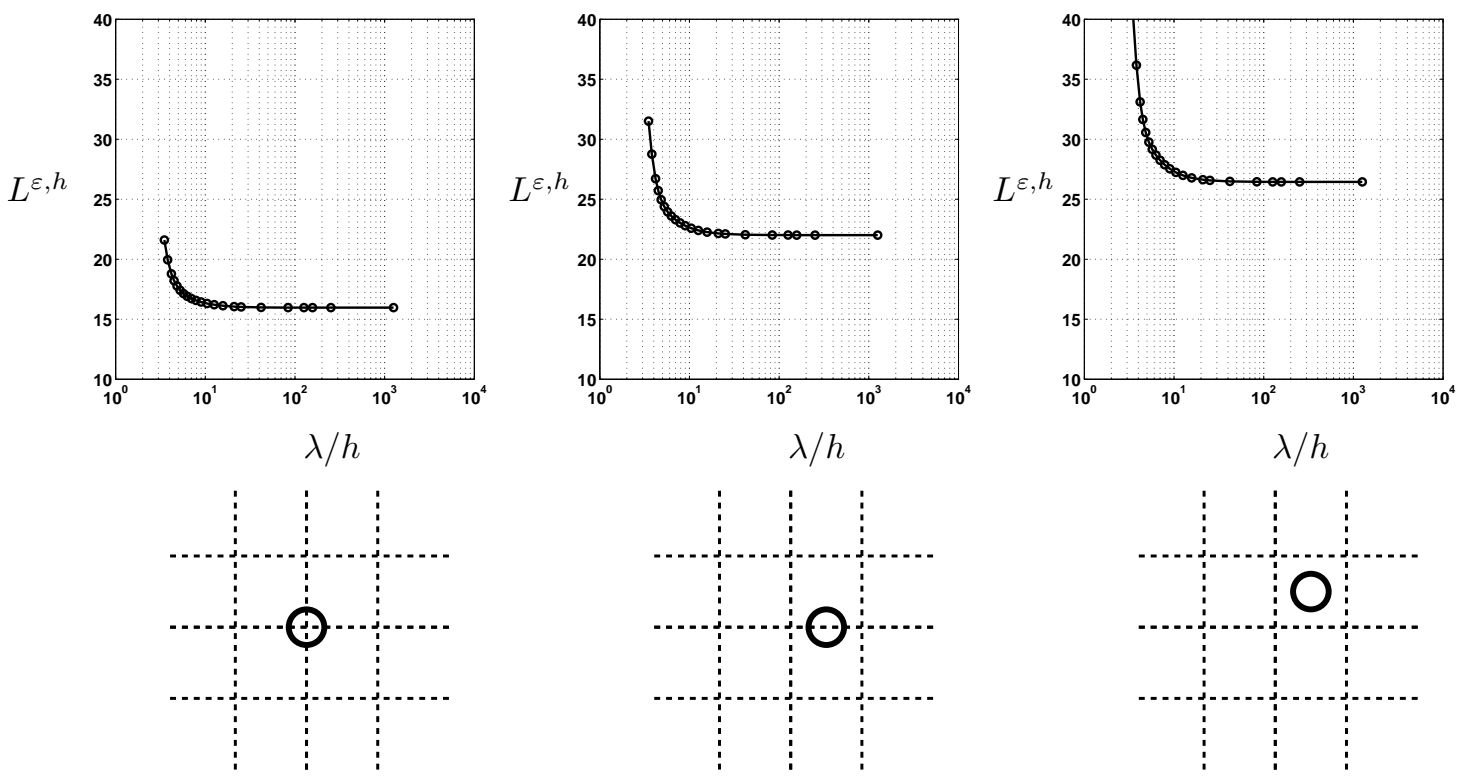

Figure 14: Dependency of the lineic inductance with respect to the location of the obstacle in the mesh.

We used a Cartesian grid with step $h / \lambda=0.65$ and an obstacle with thickness $\varepsilon / \lambda=10^{-4}$. On the lower part of Figure 14 is depicted the location of the obstacle,and the graphs in the upper part represent the corresponding dependency of the lineic inductance with respect to the wavelength. One can see that for different positions of the obstacle in the mesh there is a variation of $30 \%$ !

\section{Dependency with respect to the radius of the cut-off function}

We will show that the lineic inductance actually weakly depends on the radius of the cut-off function. Indeed consider two functions $\chi_{1}$ and $\chi_{2}$, and denote $\varphi_{1}^{\varepsilon}$ and $\varphi_{2}^{\varepsilon}$ the corresponding additional shape functions. The difference $\chi_{1}-\chi_{2}$ is very smooth and vanishes on a (fixed) neighborhood of $\Gamma^{\varepsilon}$. Thus for any $q \in \mathbb{N}$, there exists a constant $\kappa>0$ independent of $\varepsilon$ such that $\left\|\varphi_{1}^{\varepsilon}-\varphi_{2}^{\varepsilon}\right\|_{H^{q}\left(\Omega_{R}\right)} \leqslant \kappa \ln 1 / \varepsilon$. Moreover, according to (6.1), the lineic inductance only implies terms of the form $\delta_{h}\left(\varphi^{\varepsilon}\right)$, hence we conclude that the lineic inductance computed with $\varphi_{1}^{\varepsilon}$ or with $\varphi_{2}^{\varepsilon}$ is nearly the same. The bigger the neighborhood on which $\chi_{1}-\chi_{2}$ vanishes, the smaller the difference between the corresponding lineic inductance. In Figure [15, we represented the value of the Holland constant for a radius of truncation either of 0.9 or 1.2 whereas the domain of computation is a disk of radius 2 . 
Radius of truncation $=0.9$

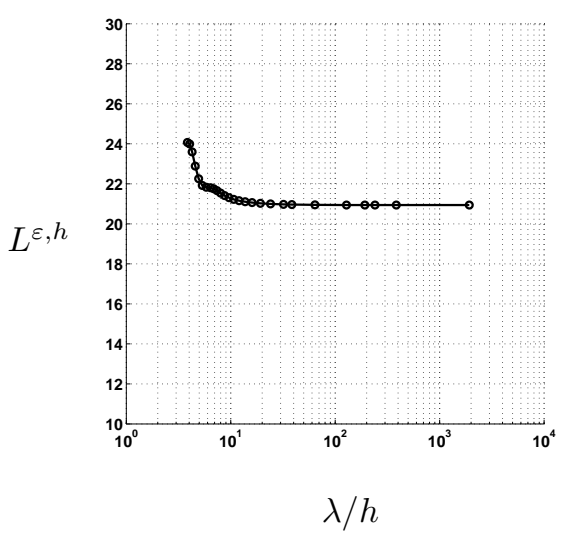

Radius of truncation $=1.2$

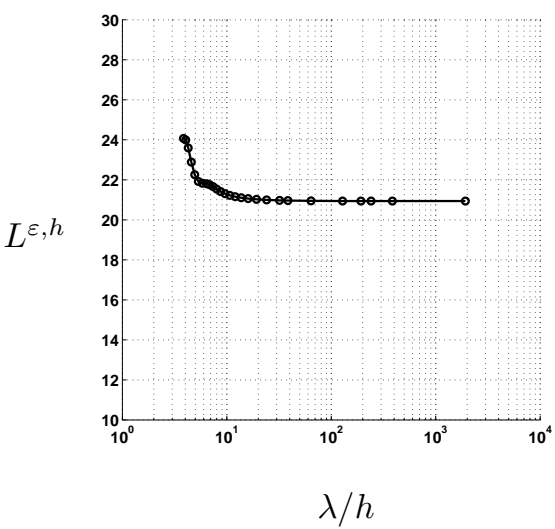

Figure 15: Dependency with respect to the radius of truncature

\subsection{Proof of numerical locking}

With the previous results, we are now able to get back to the first numerical scheme (3.1) and then prove that it suffers from real numerical locking. For this purpose, we start expressing the order of magnitude of the Lagrange multiplier that is part of the solution of the discrete problem (4.1).

\section{Lemma 6.5}

There exists a constant $\kappa>0$ independent of $\varepsilon$ and $h$ such that

$$
\left|\breve{p}^{\varepsilon, h}\right| \geqslant \frac{\kappa}{\ln 1 / \varepsilon}
$$

Proof: We start from Corollary 4.2 According to the assumptions H1, H2 and H3, we have

$$
\sqrt{\ln 1 / \varepsilon}\left(\varepsilon^{1 / 2}+h\right)=\circ\left(\frac{1}{\ln 1 / \varepsilon}\right) \text { when } h \rightarrow 0 .
$$

Moreover, on the basis of the results of [3], we can prove that there exists $\kappa>0$ such that $\left|\breve{p}^{\varepsilon}\right| \geqslant \frac{\kappa}{\ln 1 / \varepsilon}$. Finally corollary 4.2 shows that the same result holds for $\breve{p}^{\varepsilon, h}$.

Now we state the result of numerical locking.

\section{Proposition 6.1}

Suppose $u_{1}^{\varepsilon, h}$ is the solution of problem (3.1) whereas $u^{\varepsilon, h}$ is the solution of problem (4.1). Provided that assumptions $\boldsymbol{H} 1, \boldsymbol{H} 2$ are $\boldsymbol{H} 3$ are verified, there exists $\kappa>0$ independent of $\varepsilon$ and $h$ such that

$$
\left\|u^{\varepsilon}-u_{1}^{\varepsilon, h}\right\|_{H^{1}\left(\Omega_{R}\right)} \geqslant \frac{\kappa}{\ln 1 / \varepsilon}
$$

\section{Proof:}

Straightforwardly,

$$
\begin{aligned}
\left\|u^{\varepsilon}-u_{1}^{\varepsilon, h}\right\|_{H^{1}\left(\Omega_{R}\right)} & \geqslant\left\|\mathfrak{u}^{\varepsilon}-u_{1}^{\varepsilon, h}\right\|_{H^{1}\left(\Omega_{R}\right)}-\left\|u^{\varepsilon}-u^{\varepsilon, h}\right\|_{H^{1}\left(\Omega_{R}\right)} \\
& \geqslant \inf _{v^{h} \in V^{h}}\left\|\mathfrak{u}^{\varepsilon}-v^{h}\right\|_{H^{1}\left(\Omega_{R}\right)}-\left\|u^{\varepsilon}-u^{\varepsilon, h}\right\|_{H^{1}\left(\Omega_{R}\right)} \\
& \geqslant\left|p_{\mathbf{s}}^{\varepsilon, h}\right| \inf _{v^{h} \in V^{h}}\left\|\psi^{\varepsilon}-v^{h}\right\|_{H^{1}\left(\Omega_{R}\right)}-\left\|u^{\varepsilon}-u^{\varepsilon, h}\right\|_{H^{1}\left(\Omega_{R}\right)} .
\end{aligned}
$$


According to Lemma [5.2 there exists $\kappa_{2}>0$ such that $\inf _{v^{h} \in V^{h}}\left\|\psi^{\varepsilon}-v^{h}\right\|_{H^{1}\left(\Omega_{R}\right)}>\kappa_{2}$. Moreover according to corollary 4.1] there exists $\kappa_{3}>0$ such that $\left\|u^{\varepsilon}-u^{\varepsilon, h}\right\|_{H^{1}\left(\Omega_{R}\right)} \leqslant \kappa_{3} \sqrt{\ln 1 / \varepsilon}\left(\varepsilon^{1 / 2}+\right.$ $h)(\rightarrow 0)$. Thus we can write

$$
\left\|u^{\varepsilon}-u_{\mathbf{l}}^{\varepsilon, h}\right\|_{H^{1}\left(\Omega_{R}\right)} \geqslant\left|p_{\mathbf{s}}^{\varepsilon, h}\right| \kappa_{2}-\kappa_{3} \sqrt{\ln 1 / \varepsilon}\left(\varepsilon^{1 / 2}+h\right) .
$$

Using the result of Lemma 6.5 above and the fact that $\sqrt{\ln 1 / \varepsilon}\left(\varepsilon^{1 / 2}+h\right)=\circ\left(\frac{1}{\ln 1 / \varepsilon}\right)$ when $h \rightarrow 0$, we obtain the desired result.

\section{A Appendix}

\section{A.1 Nitsche and Schatz' theorem}

In this appendix, we recall a result proved by Nitsche and Schatze that allows to obtain interior estimates for finite elements schemes. We will state this result in a simplified form: we only give in this theorem what can be applied to Lagrange finite elements in two dimensions for the Helmholtz problem. This theorem is described with more details in [11].

Theorem A.1 (Nitsche and Schatz)

Consider $\Omega_{0} \subset \subset \Omega_{1} \subset \subset \mathbb{R}^{2}, u \in H^{q}\left(\Omega_{1}\right), u_{h} \in V^{h}$ (with $V^{h}=\mathbb{P}_{q}$ or $\mathbb{Q}_{q}$ ). Suppose also that

$$
a\left(u-u_{h}, v^{h}\right)=0 \quad \text { for any } \quad v^{h} \in V^{h} \quad \text { s.t } \quad \operatorname{supp} v^{h} \subset \Omega_{1}
$$

Then there exists $0<h_{1}<1$ and $\kappa>0$ such that for any $\left.\left.h \in\right] 0, h_{1}\right]$,

$$
\begin{aligned}
& \left\|u-u_{h}\right\|_{H^{1}\left(\Omega_{0}\right)} \leqslant \kappa\left(h^{q-1}\|u\|_{H^{q}\left(\Omega_{1}\right)}+\left\|u-u_{h}\right\|_{H^{-p}\left(\Omega_{1}\right)}\right) \\
& \left\|u-u_{h}\right\|_{L^{2}\left(\Omega_{0}\right)} \leqslant \kappa\left(h^{q}\|u\|_{H^{q}\left(\Omega_{1}\right)}+\left\|u-u_{h}\right\|_{H^{-p}\left(\Omega_{1}\right)}\right)
\end{aligned}
$$

\section{A.2 Technical result of geometry}

\section{Lemma A.1}

There exists $\kappa>0$ independent of $\varepsilon$ such that $\left\|\frac{\partial V_{0}^{\varepsilon}}{\partial n}\right\|_{L^{\infty}\left(\Gamma^{\varepsilon}\right)} \leqslant \frac{\kappa}{\varepsilon}$.

\section{Proof:}

Usual calculus of geometry yields the surface measure on $\Gamma^{\varepsilon}: \varepsilon\left(\gamma^{2}+\left(\frac{\partial \gamma}{\partial \theta}\right)^{2}\right)^{1 / 2}=\varepsilon \sigma(\theta)$, and the expression of its normal vector field

$$
n=n_{r} \frac{\partial}{\partial r}+n_{\theta} \frac{\partial}{\partial \theta} \quad \text { with } \quad n_{r}=\frac{\gamma}{\left(\gamma^{2}+\left(\frac{\partial \gamma}{\partial \theta}\right)^{2}\right)^{1 / 2}} \quad \text { et } \quad n_{\theta}=\frac{-\frac{\partial \gamma}{\partial \theta}}{\left(\gamma^{2}+\left(\frac{\partial \gamma}{\partial \theta}\right)^{2}\right)^{1 / 2}} .
$$

From this we immediately deduce

$$
\frac{\partial V_{0}^{\varepsilon}}{\partial n}(\mathbf{x})=\frac{1}{\sigma}\left(\gamma \frac{\partial V_{0}^{\varepsilon}}{\partial r}(\mathbf{x})-\frac{\partial \gamma}{\partial \theta} \frac{\partial V_{0}^{\varepsilon}}{\partial \theta}(\mathbf{x})\right)=\frac{1}{\varepsilon} \frac{1}{\sigma}\left(\gamma \frac{\partial V_{0}}{\partial r}\left(\frac{\mathbf{x}}{\varepsilon}\right)-\frac{\partial \gamma}{\partial \theta} \frac{\partial V_{0}}{\partial \theta}\left(\frac{\mathbf{x}}{\varepsilon}\right)\right) \quad \mathbf{x} \in \Gamma^{\varepsilon}\left(\text { i.e } \frac{\mathbf{x}}{\varepsilon} \in \Gamma\right)
$$

As there exists $\kappa>0$ independent of $\theta$ such that $\kappa<\sigma(\theta)<1 / \kappa$, we obtain a $\kappa>0$ independent of $\varepsilon$ such that

$$
\left\|\frac{\partial V_{0}^{\varepsilon}}{\partial n}\right\|_{L^{\infty}\left(\Gamma^{\varepsilon}\right)} \leqslant \frac{\kappa}{\varepsilon}\left\|\frac{\partial V_{0}}{\partial n}\right\|_{L^{\infty}(\Gamma)}
$$

which finish the proof since $V_{0} \in C^{\infty}\left(\overline{\Omega_{N}}\right)$. 


\section{A.3 Technical estimate}

\section{Lemma A.2}

There exists $\kappa>0$ independent of $\varepsilon$ such that

$$
\left\|\chi_{0}(\mathbf{x})\left(1-\chi_{1 / 2}^{\varepsilon}(\mathbf{x})\right)\left(4 V_{0}^{\varepsilon}(\mathbf{x})-\left(\frac{2}{\pi} J_{0,0} \ln \frac{|\mathbf{x}|}{\varepsilon}+Y_{0,0}+l_{0}^{1}\left(V_{0}\right)\right)\right)\right\|_{H^{1}\left(\Omega_{R}\right)} \leqslant \kappa \varepsilon^{1 / 2}
$$

\section{Proof:}

For $\varepsilon$ small enough and for $|\mathbf{x}|>\sqrt{\varepsilon} / 2, V_{0}^{\varepsilon}$ admits an expansion of the following form $(r$ and $\theta$ being the polar coordinates associated with $\mathbf{x}$ ):

$$
4 V_{0}^{\varepsilon}(\mathbf{x})-\left(\frac{2}{\pi} J_{0,0} \ln \frac{|\mathbf{x}|}{\varepsilon}+Y_{0,0}+l_{0}^{1}\left(V_{0}\right)\right)=\sum_{|p| \geqslant 1} V_{0, p} \frac{\varepsilon^{|p|}}{r^{|p|}} e^{i p \theta} .
$$

Then

$$
\begin{gathered}
\left\|\chi_{0}(\mathbf{x})\left(1-\chi_{1 / 2}^{\varepsilon}(\mathbf{x})\right)(\mathbf{x})\left(4 V_{0}^{\varepsilon}(\mathbf{x})-\left(\frac{2}{\pi} J_{0,0} \ln \frac{|\mathbf{x}|}{\varepsilon}+Y_{0,0}+l_{0}^{1}\left(V_{0}\right)\right)\right)\right\|_{H^{1}\left(\Omega_{R}\right)}^{2} \\
\leqslant \kappa \sum_{|p| \geqslant 1}\left(1+p^{2}\right)\left|V_{0, p}\right|^{2} \varepsilon^{2|p|}\left\|\frac{1-\chi_{1 / 2}^{\varepsilon}}{r|p|}\right\|_{H^{1}\left(\Omega_{R}\right)}^{2} .
\end{gathered}
$$

A short calculus shows that

$$
\begin{aligned}
\varepsilon^{2|p|}\left\|\frac{1-\chi_{1 / 2}^{\varepsilon}}{r^{|p|}}\right\|_{H^{1}\left(\Omega_{R}\right)}^{2} & \leqslant \kappa \varepsilon^{2|p|} \int_{\sqrt{\varepsilon} / 2}^{R} \frac{d r}{r^{2|p|-1}}+\kappa \varepsilon^{2|p|-1} \int_{\sqrt{\varepsilon} / 2}^{\sqrt{\varepsilon}} \frac{d r}{r^{2}|p|-1}+\kappa p^{2} \varepsilon^{2|p|} \int_{\sqrt{\varepsilon} / 2}^{R} \frac{d r}{r^{2}|p|+1} \\
& \leqslant \kappa \varepsilon
\end{aligned}
$$

As the series $\sum_{|p| \geqslant 1}\left(1+p^{2}\right)\left|V_{0, p}\right|^{2}$ converges absolutely, we get the result of this lemma.

\section{Lemma A.3}

Provided that $V^{h}$ is an approximation space associated with finite elements of order $q$, there exists $\kappa>0$ independent of $\varepsilon$ and $h$ such that

$$
\inf _{w_{h} \in V^{h}}\left\|\widetilde{u}^{\varepsilon}-\frac{-\frac{\pi}{2} u_{0}(0)}{\ln \varepsilon-\lambda_{0}} \widetilde{\psi}^{\varepsilon}-w_{h}\right\|_{H^{1}\left(\Omega_{R}\right)} \leqslant \kappa\left(\varepsilon^{1 / 2}+h^{q}\right)
$$

\section{Proof:}

We recall the expression of $\mathfrak{u}^{\varepsilon}$,

$$
\begin{aligned}
\widetilde{u}^{\varepsilon}(\mathbf{x}) & =\left(1-\chi_{1 / 2}^{\varepsilon}(\mathbf{x})\right)\left(u_{0}(\mathbf{x})+\frac{-\frac{\pi}{2} u_{0}(0)}{\ln \varepsilon-\lambda_{0}} v_{0}(\mathbf{x})\right)+\chi_{1 / 2}^{\varepsilon}(\mathbf{x}) \frac{-\frac{\pi}{2} u_{0}(0)}{\ln \varepsilon-\lambda_{0}} V_{0}^{\varepsilon}(\mathbf{x}) \mathbf{1}_{\Omega_{R} \backslash \bar{\Omega}_{\varepsilon}^{i}}(\mathbf{x}) \\
& =\left(1-\chi_{1 / 2}^{\varepsilon}(\mathbf{x})\right)\left(u_{0}(\mathbf{x})-u_{0}(0)+\frac{-\frac{\pi}{2} u_{0}(0)}{\ln \varepsilon-\lambda_{0}}\left(v_{0}(\mathbf{x})+\frac{2}{\pi}\left(\ln 1 / \varepsilon+\lambda_{0}\right)\right)\right) \\
& +\chi_{1 / 2}^{\varepsilon}(\mathbf{x}) \frac{-\frac{\pi}{2} u_{0}(0)}{\ln \varepsilon-\lambda_{0}} V_{0}^{\varepsilon}(\mathbf{x}) \mathbf{1}_{\Omega_{R} \backslash \bar{\Omega}_{\varepsilon}^{i}}(\mathbf{x})
\end{aligned}
$$

Note that

$$
\begin{aligned}
v_{0}(\mathbf{x}) & =Y_{0}(k|\mathbf{x}|)+\mathcal{L}_{0}^{1}\left(v_{0}\right) J_{0}(k|\mathbf{x}|)=\widetilde{Y}_{0}(k|\mathbf{x}|)+\frac{2}{\pi} J_{0}(k|\mathbf{x}|) \ln |\mathbf{x}|+\mathcal{L}_{0}^{1}\left(v_{0}\right) J_{0}(k|\mathbf{x}|) \\
& =\widetilde{Y}_{0}(k|\mathbf{x}|)+\mathcal{L}_{0}^{1}\left(v_{0}\right) J_{0}(k|\mathbf{x}|)+\frac{2}{\pi}\left(J_{0}(k|\mathbf{x}|)-1\right) \ln |\mathbf{x}|+\frac{2}{\pi} \ln |\mathbf{x}|
\end{aligned}
$$




$$
\begin{aligned}
v_{0}(\mathbf{x})+ & \frac{2}{\pi}\left(\ln 1 / \varepsilon+\lambda_{0}\right)=\left(\widetilde{Y}_{0}(k|\mathbf{x}|)-Y_{0,0}\right)+\mathcal{L}_{0}^{1}\left(v_{0}\right)\left(J_{0}(k|\mathbf{x}|)-1\right) \\
& +\frac{2}{\pi}\left(J_{0}(k|\mathbf{x}|)-1\right) \ln |\mathbf{x}|+\frac{2}{\pi} J_{0,0} \ln \frac{|\mathbf{x}|}{\varepsilon}+Y_{0,0}+l_{0}^{1}\left(V_{0}\right) \\
\widetilde{u}^{\varepsilon}(\mathbf{x})= & \left(1-\chi_{1 / 2}^{\varepsilon}(\mathbf{x})\right)\left(u_{0}(\mathbf{x})-u_{0}(0)\right)+\left(1-\chi_{1 / 2}^{\varepsilon}(\mathbf{x})\right) \frac{-\frac{\pi}{2} u_{0}(0)}{\ln \varepsilon-\lambda_{0}}\left(\widetilde{Y}_{0}(k|\mathbf{x}|)-Y_{0,0}\right) \\
+ & \left(1-\chi_{1 / 2}^{\varepsilon}(\mathbf{x})\right) \frac{-\frac{\pi}{2} u_{0}(0)}{\ln \varepsilon-\lambda_{0}}\left(\mathcal{L}_{0}^{1}\left(v_{0}\right)\left(J_{0}(k|\mathbf{x}|)-1\right)+\frac{2}{\pi}\left(J_{0}(k|\mathbf{x}|)-1\right) \ln |\mathbf{x}|\right) \\
+ & \left(1-\chi_{1 / 2}^{\varepsilon}(\mathbf{x})\right) \frac{-\frac{\pi}{2} u_{0}(0)}{\ln \varepsilon-\lambda_{0}}\left(\frac{2}{\pi} J_{0,0} \ln \frac{|\mathbf{x}|}{\varepsilon}+Y_{0,0}+l_{0}^{1}\left(V_{0}\right)\right) \\
+ & \chi_{1 / 2}^{\varepsilon}(\mathbf{x}) \frac{-\frac{\pi}{2} u_{0}(0)}{\ln \varepsilon-\lambda_{0}} V_{0}^{\varepsilon}(\mathbf{x}) \mathbf{1}_{\Omega_{R} \backslash \bar{\Omega}_{\varepsilon}^{i}}(\mathbf{x}) \\
= & \left(1-\chi_{1 / 2}^{\varepsilon}(\mathbf{x})\right)\left(u_{0}(\mathbf{x})-u_{0}(0)\right)+\left(1-\chi_{1 / 2}^{\varepsilon}(\mathbf{x})\right) \frac{-\frac{\pi}{2} u_{0}(0)}{\ln \varepsilon-\lambda_{0}}\left(\widetilde{Y}_{0}(k|\mathbf{x}|)-Y_{0,0}\right) \\
+ & \left(1-\chi_{1 / 2}^{\varepsilon}(\mathbf{x})\right) \frac{-\frac{\pi}{2} u_{0}(0)}{\ln \varepsilon-\lambda_{0}}\left(\mathcal{L}_{0}^{1}\left(v_{0}\right)\left(J_{0}(k|\mathbf{x}|)-1\right)+\frac{2}{\pi}\left(J_{0}(k|\mathbf{x}|)-1\right) \ln |\mathbf{x}|\right) \\
& +\mathbf{1}_{\Omega_{R} \backslash \bar{\Omega}_{\varepsilon}^{i}(\mathbf{x}) \frac{-\frac{\pi}{2}}{\ln \varepsilon-\lambda_{0}}(0)}\left(\frac{2}{\pi} J_{0,0} \ln \frac{|\mathbf{x}|}{\varepsilon}+Y_{0,0}+l_{0}^{1}\left(V_{0}\right)\right) \\
& +\chi_{1 / 2}^{\varepsilon}(\mathbf{x}) \mathbf{1}_{\Omega_{R} \backslash \bar{\Omega}_{\varepsilon}^{i}}(\mathbf{x}) \frac{-\frac{\pi}{2} u_{0}(0)}{\ln \varepsilon-\lambda_{0}}\left(V_{0}^{\varepsilon}(\mathbf{x})-\left(\frac{2}{\pi} J_{0,0} \ln \frac{|\mathbf{x}|}{\varepsilon}+Y_{0,0}+l_{0}^{1}\left(V_{0}\right)\right)\right)
\end{aligned}
$$

Note that the last three terms of the preceding sum match the definition of $\widetilde{\psi}_{\text {ad }}^{\varepsilon}$ so a simple calculus shows that

$$
\begin{aligned}
\widetilde{u}^{\varepsilon}(\mathbf{x})-\frac{-\frac{\pi}{2}}{\ln \varepsilon} & -\lambda_{0}(0) \widetilde{\psi}^{\varepsilon}(\mathbf{x}) \\
= & \underbrace{\left(1-\chi_{1 / 2}^{\varepsilon}(\mathbf{x})\right)\left(u_{0}(\mathbf{x})-u_{0}(0)\right)}_{=T_{1}^{\varepsilon}(\mathbf{x})}+\underbrace{\left(1-\chi_{1 / 2}^{\varepsilon}(\mathbf{x})\right) \frac{-\frac{\pi}{2} u_{0}(0)}{\ln \varepsilon-\lambda_{0}}\left(\widetilde{Y}_{0}(k|\mathbf{x}|)-Y_{0,0}\right)}_{=T_{2}^{\varepsilon}(\mathbf{x})} \\
& +\underbrace{\left(1-\chi_{1 / 2}^{\varepsilon}(\mathbf{x})\right) \frac{-\frac{\pi}{2} u_{0}(0)}{\ln \varepsilon-\lambda_{0}} \mathcal{L}_{0}^{1}\left(v_{0}\right)\left(J_{0}(k|\mathbf{x}|)-1\right)}_{=T_{3}^{\varepsilon}(\mathbf{x})} \\
& +\underbrace{\left(1-\chi_{0}(\mathbf{x})\right) \frac{-\frac{\pi}{2} u_{0}(0)}{\ln \varepsilon-\lambda_{0}} \frac{2}{\pi}\left(J_{0}(k|\mathbf{x}|)-1\right) \ln |\mathbf{x}|}_{=T_{4}^{\varepsilon}(\mathbf{x})} \\
& +\underbrace{\left(1-\chi_{0}(\mathbf{x})\right) \frac{-\frac{\pi}{2} u_{0}(0)}{\ln \varepsilon-\lambda_{0}}\left(\frac{2}{\pi} J_{0,0} \ln \frac{|\mathbf{x}|}{\varepsilon}+Y_{0,0}+l_{0}^{1}\left(V_{0}\right)\right)}_{=T_{5}^{\varepsilon}(\mathbf{x})}
\end{aligned}
$$

We will finally provide an upper bound for each of the terms $T_{i}^{\varepsilon}$ using Strang lemma.

- Estimation of $\left\|T_{1}^{\varepsilon}-w_{h}\right\|_{H^{1}\left(\Omega_{R}\right)}$

$$
\begin{aligned}
\inf _{w_{h} \in V_{e}^{h}}\left\|T_{1}^{\varepsilon}-w_{h}\right\|_{H^{1}\left(\Omega_{R}\right)} & \leqslant \inf _{w_{h} \in V_{e}^{h}}\left\|u_{0}-u_{0}(0)-w_{h}\right\|_{H^{1}\left(\Omega_{R}\right)}+\left\|\chi_{1 / 2}^{\varepsilon}\left(u_{0}-u_{0}(0)\right)\right\|_{H^{1}\left(\Omega_{R}\right)} \\
& \leqslant \kappa\left(h^{q}+\varepsilon^{1 / 2}\right) .
\end{aligned}
$$


- Estimation of $\left\|T_{2}^{\varepsilon}-w_{h}\right\|_{H^{1}\left(\Omega_{R}\right)}$

$$
\begin{aligned}
\inf _{w_{h} \in V_{e}^{h}}\left\|T_{2}^{\varepsilon}-w_{h}\right\|_{H^{1}\left(\Omega_{R}\right)} \leqslant & \frac{\kappa}{\ln 1 / \varepsilon}\left(\inf _{w_{h} \in V_{e}^{h}}\left\|\widetilde{Y}_{0}(k|\cdot|)-Y_{0,0}-w_{h}\right\|_{H^{1}\left(\Omega_{R}\right)}\right. \\
& \left.+\left\|\chi_{1 / 2}^{\varepsilon}\left(\widetilde{Y}_{0}(k|\cdot|)-Y_{0,0}\right)\right\|_{H^{1}\left(\Omega_{R}\right)}\right) \\
\leqslant & \frac{\kappa}{\ln 1 / \varepsilon}\left(h^{q}+\varepsilon^{1 / 2}\right)
\end{aligned}
$$

- Estimation of $\left\|T_{3}^{\varepsilon}-w_{h}\right\|_{H^{1}\left(\Omega_{R}\right)}$

$$
\begin{aligned}
\inf _{w_{h} \in V_{e}^{h}}\left\|T_{3}^{\varepsilon}-w_{h}\right\|_{H^{1}\left(\Omega_{R}\right)} \leqslant \frac{\kappa}{\ln 1 / \varepsilon}\left(\inf _{w_{h} \in V_{e}^{h}}\left\|J_{0}(k|\cdot|)-1-w_{h}\right\|_{H^{1}\left(\Omega_{R}\right)}\right. \\
\left.+\left\|\chi_{1 / 2}^{\varepsilon}\left(J_{0}(k|\cdot|)-1\right)\right\|_{H^{1}\left(\Omega_{R}\right)}\right) \\
\leqslant \frac{\kappa}{\ln 1 / \varepsilon}\left(h^{q}+\varepsilon^{1 / 2}\right)
\end{aligned}
$$

- Estimation of $\left\|T_{4}^{\varepsilon}-w_{h}\right\|_{H^{1}\left(\Omega_{R}\right)}$

$$
\begin{gathered}
\inf _{w_{h} \in V_{e}^{h}}\left\|T_{4}^{\varepsilon}-w_{h}\right\|_{H^{1}\left(\Omega_{R}\right)} \leqslant \frac{\kappa}{\ln 1 / \varepsilon} \inf _{w_{h} \in V_{e}^{h}}\left\|\left(1-\chi_{0}(\mathbf{x})\right)\left(J_{0}(k|\mathbf{x}|)-1\right) \ln |\mathbf{x}|-w_{h}\right\|_{H^{1}\left(\Omega_{R}\right)} \\
\leqslant \frac{\kappa}{\ln 1 / \varepsilon} h^{q}
\end{gathered}
$$

- Estimation of $\left\|T_{5}^{\varepsilon}-w_{h}\right\|_{H^{1}\left(\Omega_{R}\right)}$

$$
\begin{aligned}
\inf _{w_{h} \in V_{e}^{h}} \| T_{4}^{\varepsilon} & -w_{h} \|_{H^{1}\left(\Omega_{R}\right)} \\
& \leqslant \frac{\kappa}{\ln 1 / \varepsilon} \inf _{w_{h} \in V_{e}^{h}}\left\|\left(1-\chi_{0}(\mathbf{x})\right)\left(\frac{2}{\pi} J_{0,0} \ln \frac{|\mathbf{x}|}{\varepsilon}+Y_{0,0}+l_{0}^{1}\left(V_{0}\right)\right)-w_{h}\right\|_{H^{1}\left(\Omega_{R}\right)} \\
& \leqslant \kappa h^{q}
\end{aligned}
$$

and the lemma is proved, since

$$
\begin{aligned}
& \quad \inf _{w_{h} \in V^{h}}\left\|T_{1}^{\varepsilon}+T_{2}^{\varepsilon}+T_{3}^{\varepsilon}+T_{4}^{\varepsilon}+T_{5}^{\varepsilon}-w_{h}\right\|_{H^{1}\left(\Omega_{R}\right)} \leqslant \\
& \quad \inf _{w_{h} \in V^{h}}\left\|T_{1}^{\varepsilon}-w_{h}\right\|_{H^{1}\left(\Omega_{R}\right)}+\inf _{w_{h} \in V^{h}}\left\|T_{2}^{\varepsilon}-w_{h}\right\|_{H^{1}\left(\Omega_{R}\right)} \\
& +\inf _{w_{h} \in V^{h}}\left\|T_{3}^{\varepsilon}-w_{h}\right\|_{H^{1}\left(\Omega_{R}\right)}+\inf _{w_{h} \in V^{h}}\left\|T_{4}^{\varepsilon}-w_{h}\right\|_{H^{1}\left(\Omega_{R}\right)}+\inf _{w_{h} \in V^{h}}\left\|T_{5}^{\varepsilon}-w_{h}\right\|_{H^{1}\left(\Omega_{R}\right)}
\end{aligned}
$$

\section{Oh !...Un camion !}

\section{References}

[1] F. Brezzi and M. Fortin. Mixed and Finite Element Methods. Springer-Verlag, 1991.

[2] P. Ciarlet, Jr., B. Jung, S. Kaddouri, S. Labrunie, and J. Zou. The Fourier singular complement method for the Poisson problem. I. Prismatic domains. Numer. Math., 101(3):423-450, 2005 . 
[3] X. Claeys, H. Haddar, and P. Joly. Etude d'un problème modèle pour la diffraction par des fils minces par développements asymptotiques raccordés. cas 2-d. Research Report 5839, INRIA, 2006.

[4] F. Collino and F. Millot. Fils et méthodes d' éléments finis pour les équations de maxwell. le modèle de holland revisité. Technical Report 3472, Inria, 1998.

[5] F Collino, F. Millot, and P. Joly. Fictitious domain method for unsteady problems: application to electromagnetic scattering. J.Comput.Phys, 138(2):907-938, 1997.

[6] M. Duruflé. Intégration numérique et éléments finis d'ordre élevé appliqués aux équations de Maxwell en régimeharmonique. PhD thesis, Université Paris Dauphine, 2006.

[7] V. Girault and P.A. Raviart. Finite element method for Navier-Stokes equations. SpringerVerlag, 1986.

[8] R. Holland and L. Simpson. Finite-difference analysis of emp coupling to thin struts and wires. IEEE Trans. Electromagn. Compat., 1981.

[9] E. Jamelot. Résolution des équation de Maxwell avec des éléments finis de Galerkin continus. $\mathrm{PhD}$ thesis, Ecole Polytechnique, Septembre 2006.

[10] N. Lebedev. Special Functions and their applications. Dover Publications, 1972.

[11] J.A. Nitsche and A.H. Schatz. Interior estimates for ritz-galerkin methods. Mathematics of Computation, 28(128):937-958, October 1974.

[12] F. Rogier. Problèmes mathématiques et numériques lies à l'approximation de la géométrie d'un corps diffractant dans les équations de l'électromagnétisme. PhD thesis, Paris VI, 1989. 


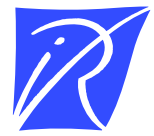

Unité de recherche INRIA Rocquencourt

Domaine de Voluceau - Rocquencourt - BP 105 - 78153 Le Chesnay Cedex (France)

Unité de recherche INRIA Futurs : Parc Club Orsay Université - ZAC des Vignes

4, rue Jacques Monod - 91893 ORSAY Cedex (France)

Unité de recherche INRIA Lorraine : LORIA, Technopôle de Nancy-Brabois - Campus scientifique

615, rue du Jardin Botanique - BP 101 - 54602 Villers-lès-Nancy Cedex (France)

Unité de recherche INRIA Rennes : IRISA, Campus universitaire de Beaulieu - 35042 Rennes Cedex (France)

Unité de recherche INRIA Rhône-Alpes : 655, avenue de l'Europe - 38334 Montbonnot Saint-Ismier (France)

Unité de recherche INRIA Sophia Antipolis : 2004, route des Lucioles - BP 93 - 06902 Sophia Antipolis Cedex (France)

INRIA - Domaine de Voluceau - Rocquencourt, BP 105 - 78153 Le Chesnay Cedex (France)

http://www.inria.fr

ISSN 0249-6399 